\title{
Orthoesters Formation Leading to Mismatched Helferich Glycosylations at O-3 of $N$-trichloroacetylated Glucosamine Residues.
}

\author{
Adam Forman and France-Isabelle Auzanneau* \\ Department of Chemistry, University of Guelph, Guelph, Ontario, N1G 2WI, Canada \\ Corresponding author. Tel: 01-519-824-4120, ext. 53809; fax: 01-519-766-1499; \\ e-mail: fauzanne@uoguelph.ca
}

\section{Supplementary Material}

Table of Contents

1. ${ }^{1} \mathrm{H}$ - and ${ }^{13} \mathrm{C}$ - NMR spectra for compounds 9-24, 26-28

2. Scheme describing the synthesis of diol acceptors $\mathbf{3}$ and $\mathbf{2 5}$ via intermediates $\mathbf{3 0}, \mathbf{3 1}$

3. Experimental procedures for the synthesis of compounds $3,25, \mathbf{3 0}, 31$

79

4. ${ }^{1} \mathrm{H}$ - and ${ }^{13} \mathrm{C}$ - NMR spectra for compounds $3,25,30,31$

$80-83$

$84-100$ 
1. NMR data for compounds 9-24, 26-28 
Compound 9, $400 \mathrm{MHz}, \mathrm{CDCl}_{3}$

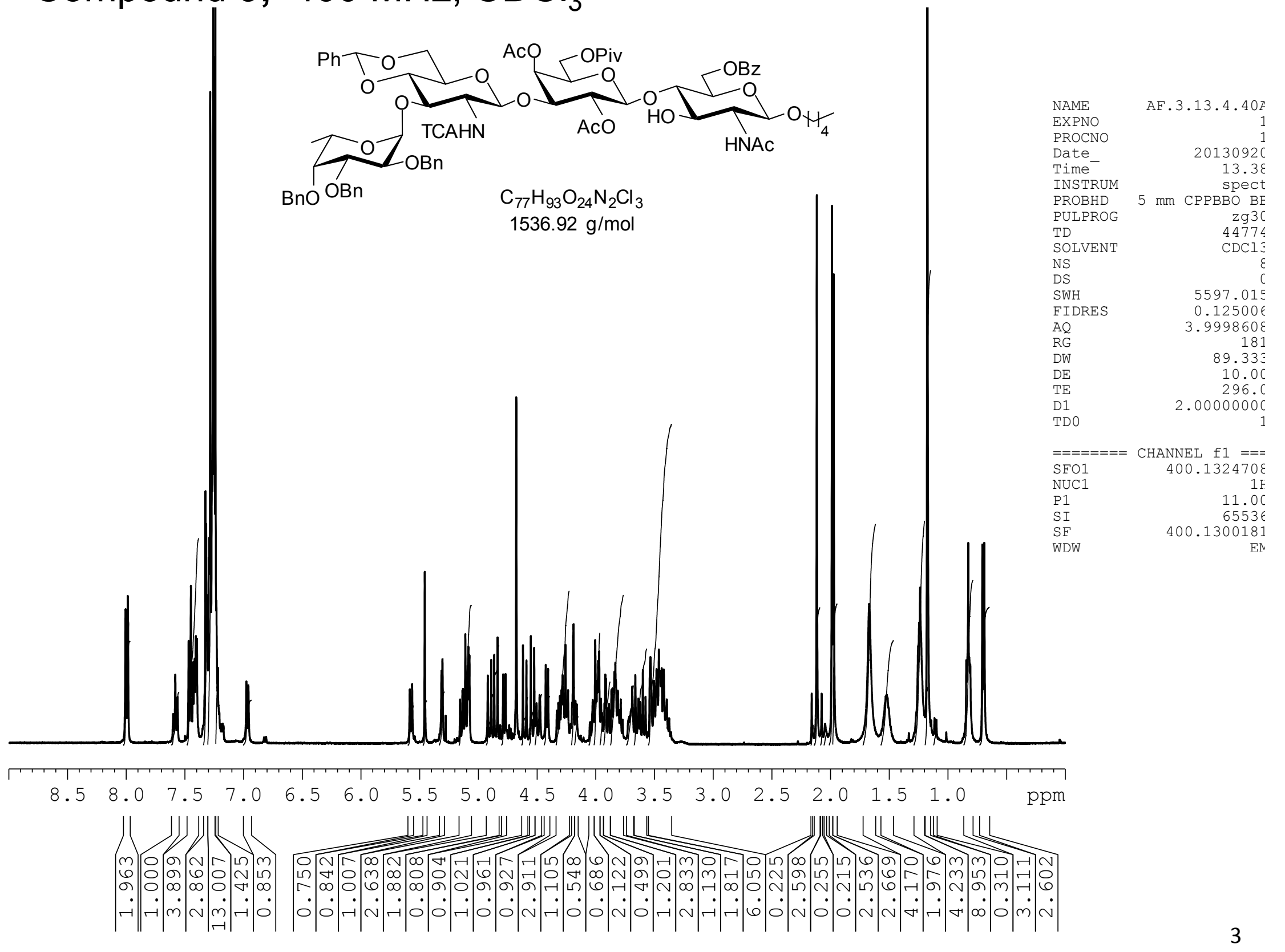




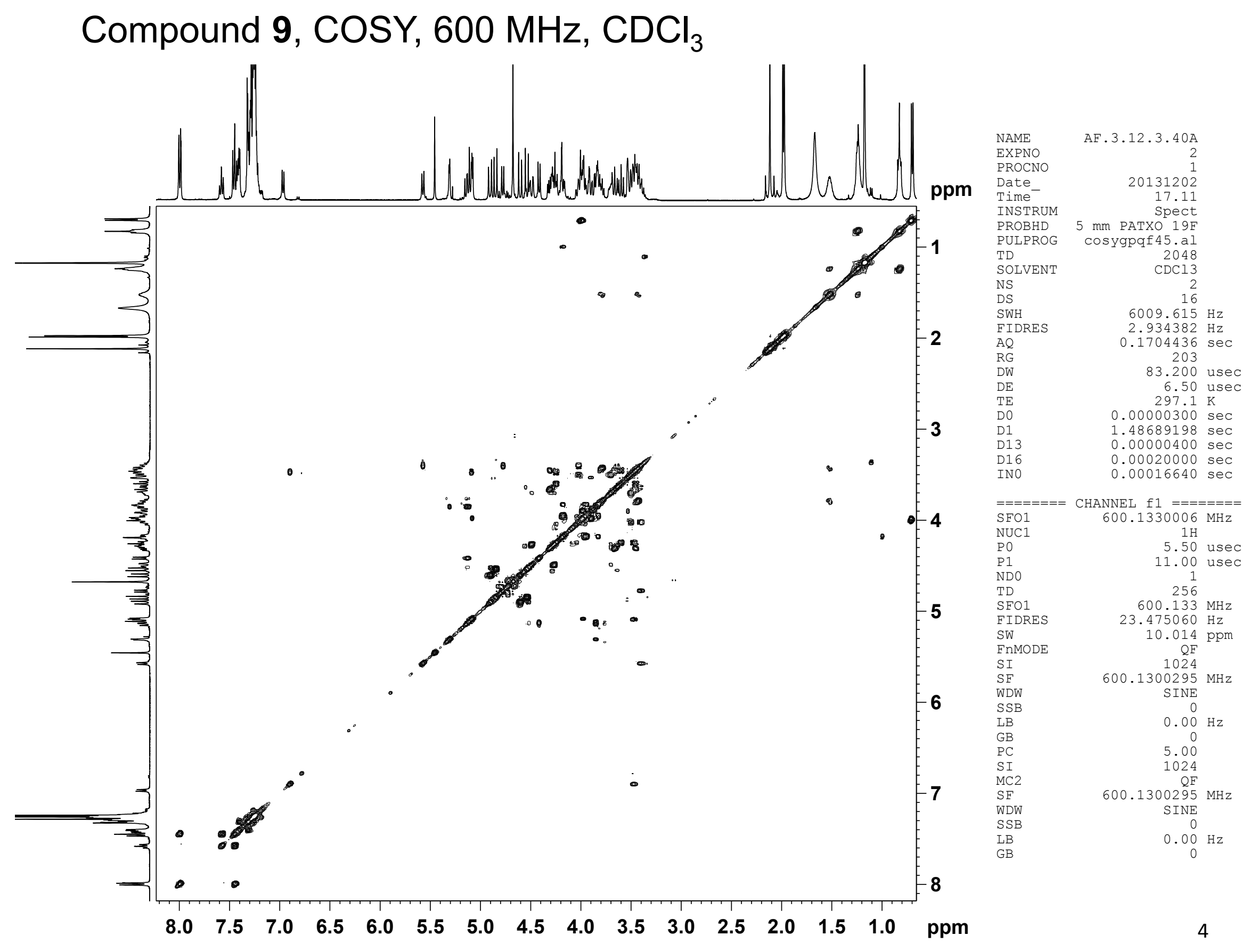




\section{Compound 9, $151 \mathrm{MHz}, \mathrm{CDCl}_{3}$}

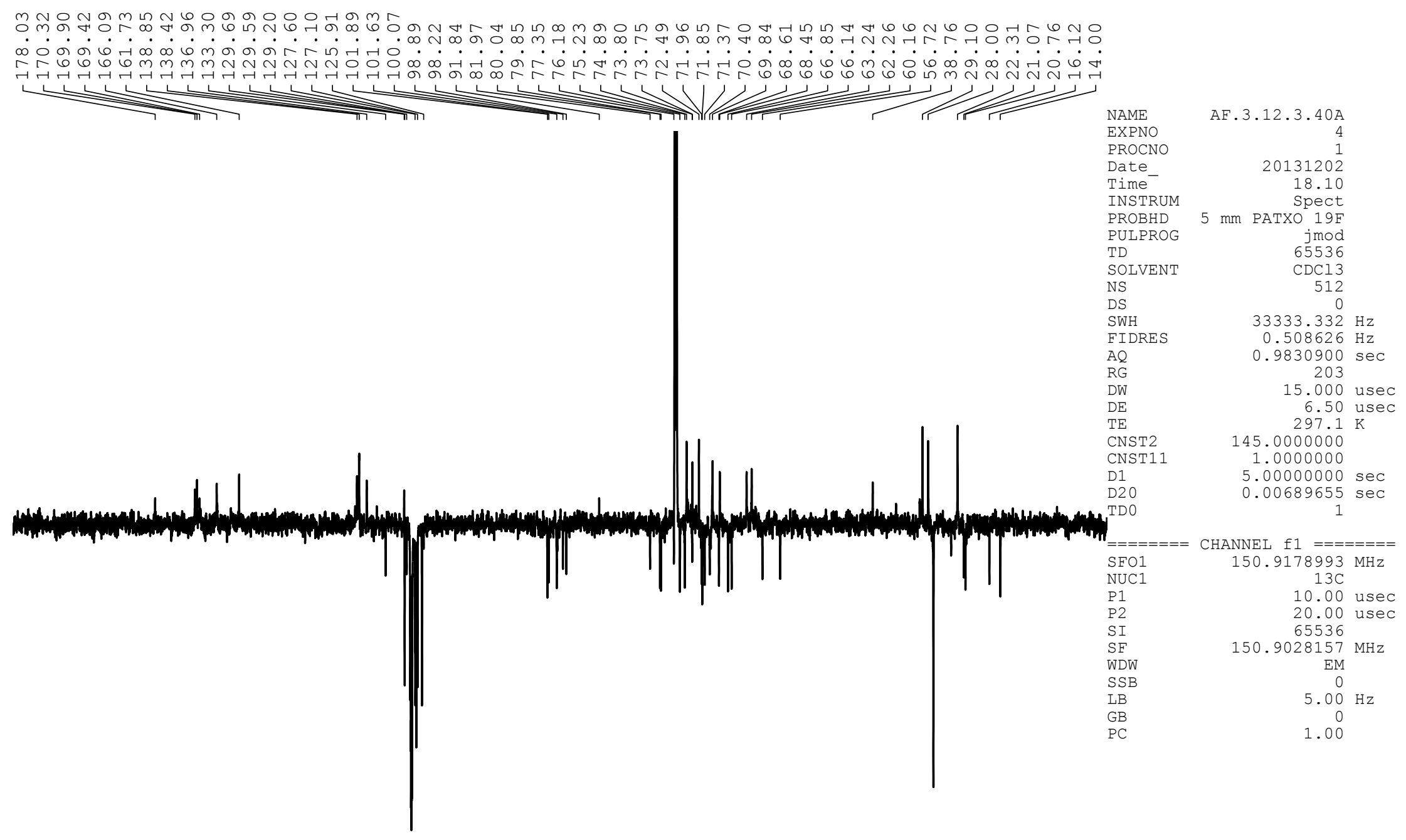

\begin{tabular}{lrrrrrrrrr|r}
\hline 200 & 180 & 160 & 140 & 120 & 100 & 80 & 60 & 40 & 20 & ppm
\end{tabular}


Compound 9, edited HSQC ( $\mathrm{CH}$ and $\mathrm{CH}_{3}$ blue, $\mathrm{CH}_{2}$ black), $600 \mathrm{MHz}, \mathrm{CDCl}_{3}$

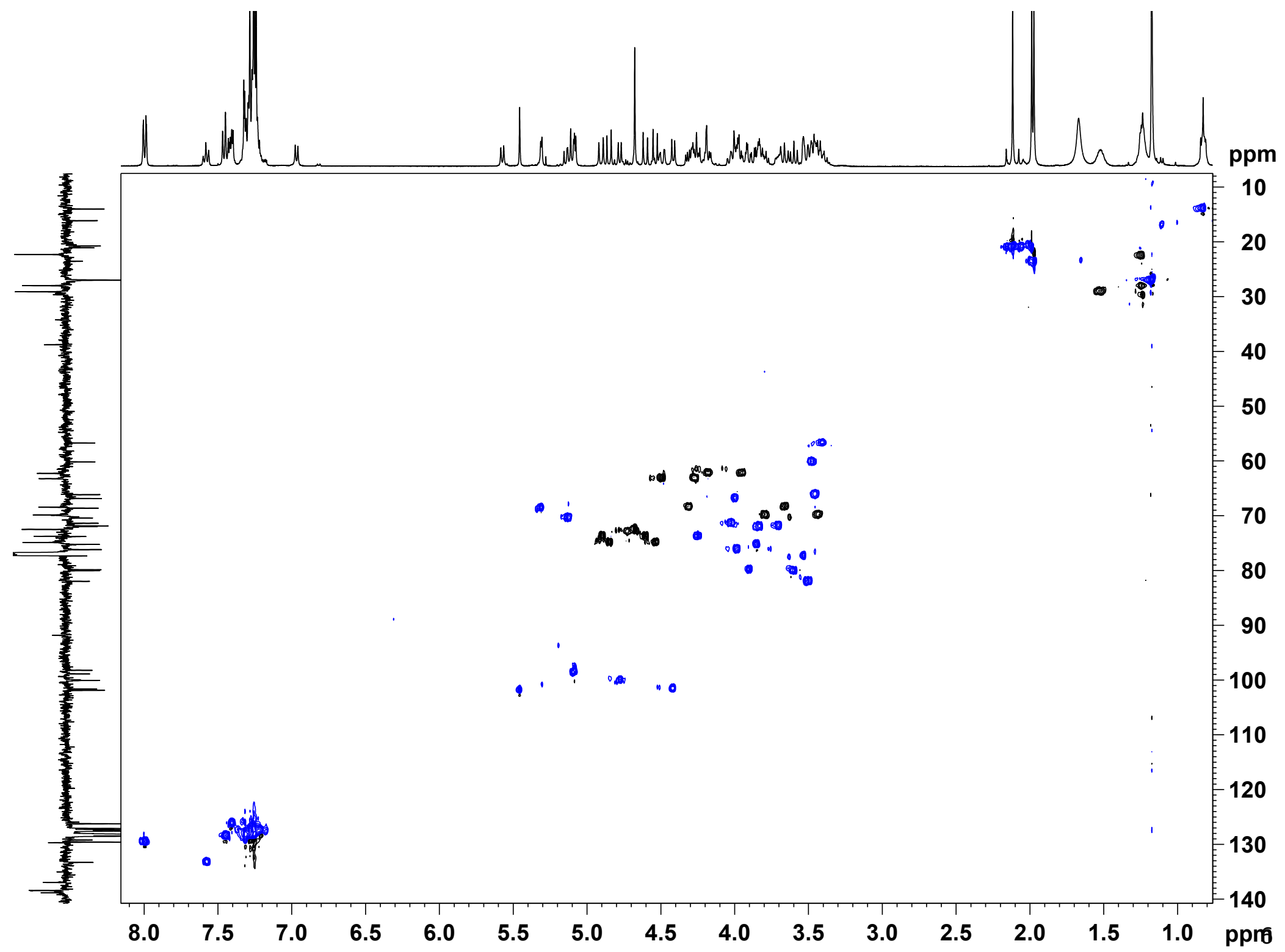


Compound 10, $400 \mathrm{MHz}, \mathrm{CDCl}_{3}$
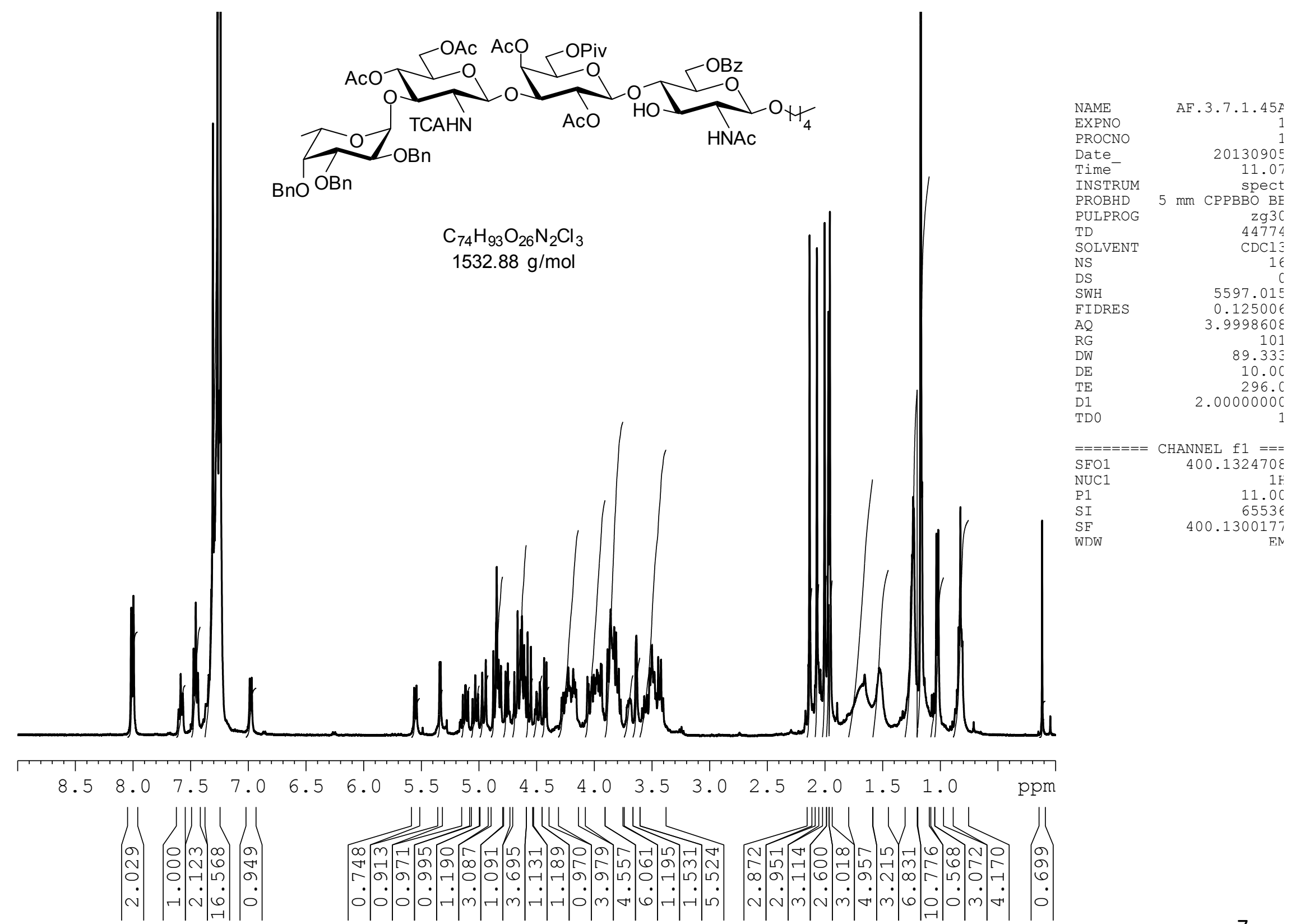
Compound 10, COSY, $400 \mathrm{MHz}, \mathrm{CDCl}_{3}$

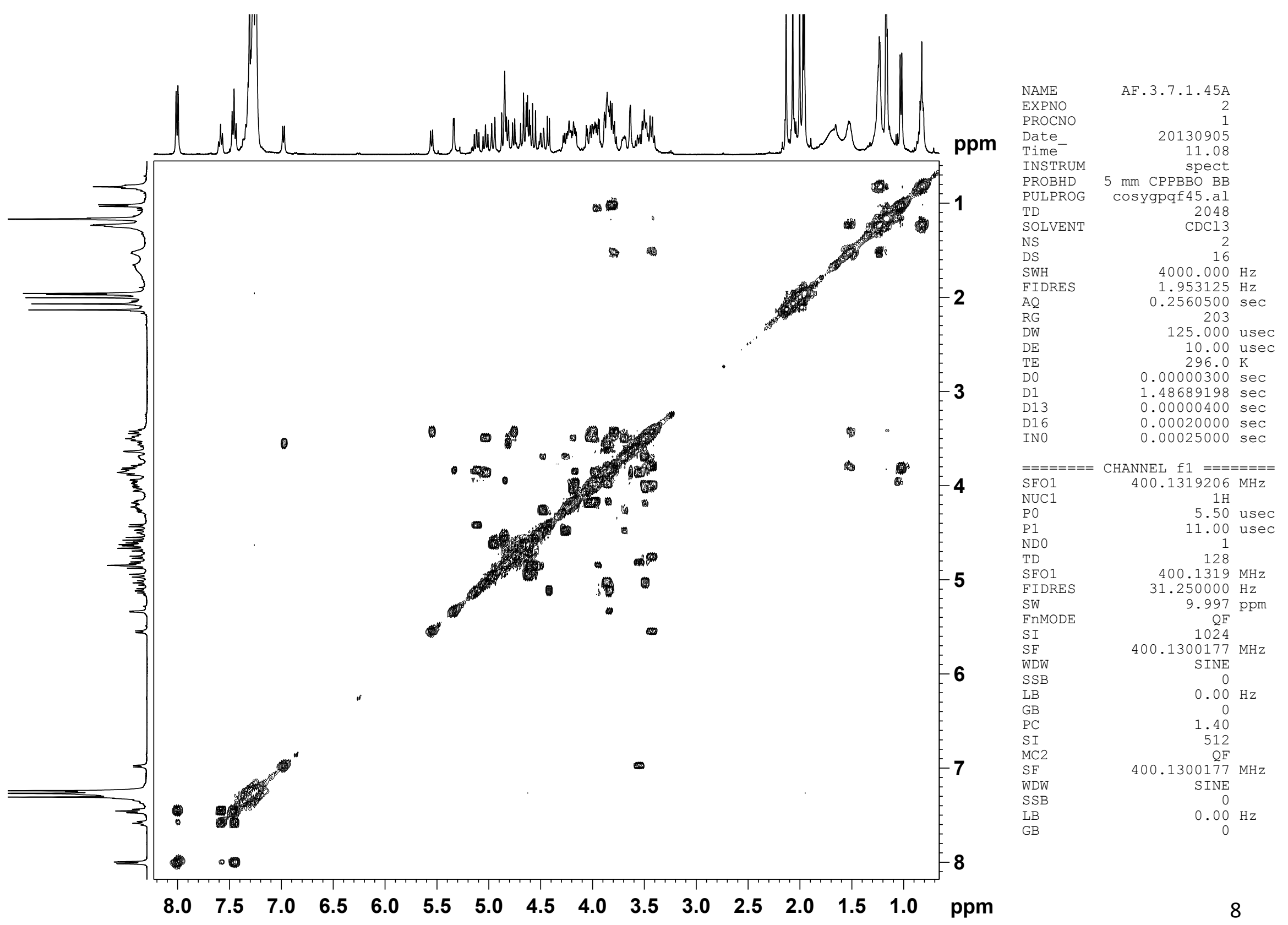




\section{Compound 10, $101 \mathrm{MHz}, \mathrm{CDCl}_{3}$}

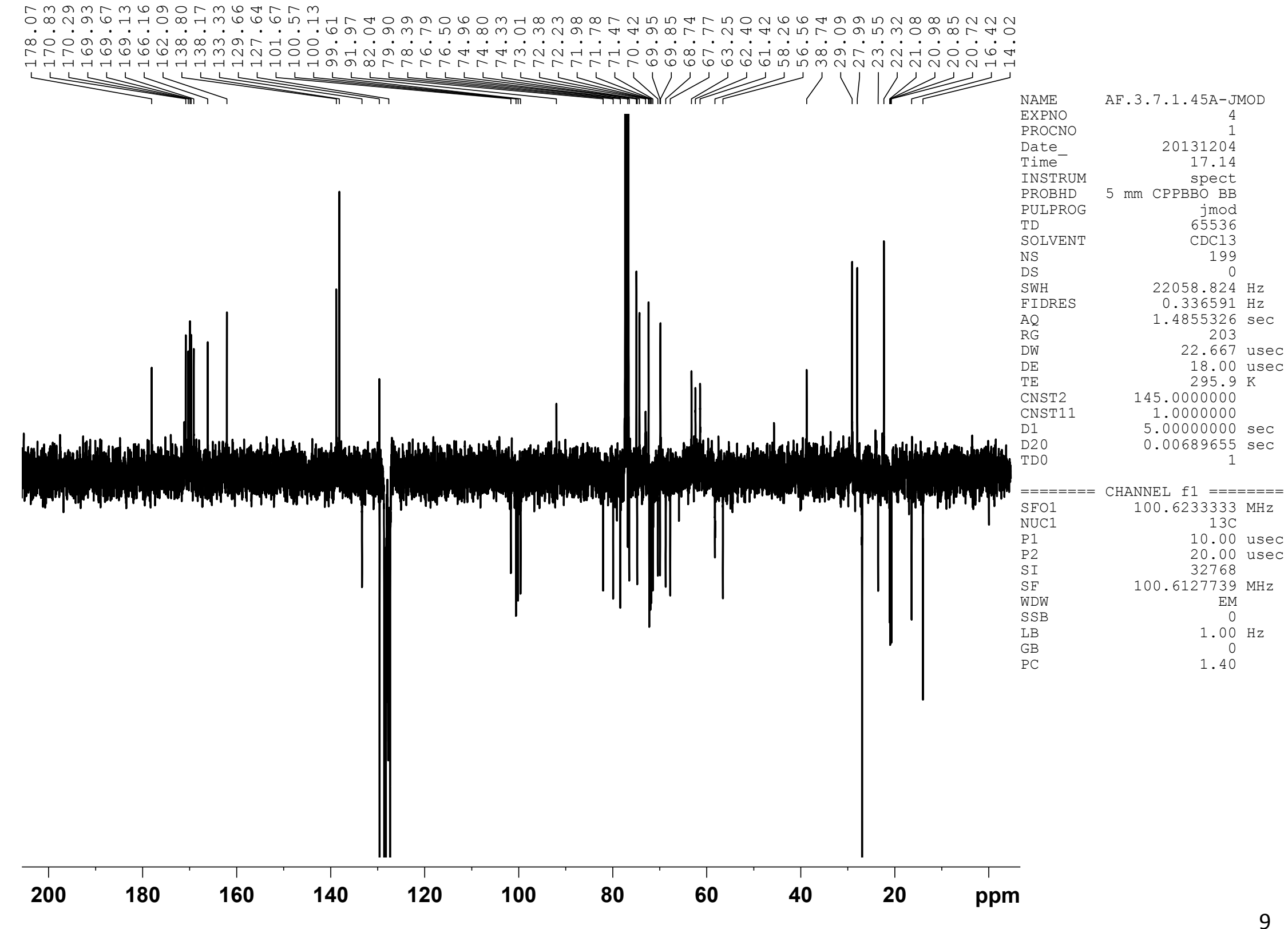


Compound 10, edited $\mathrm{HSQC}\left(\mathrm{CH}\right.$ and $\mathrm{CH}_{3}$ blue, $\mathrm{CH}_{2}$ black), $400 \mathrm{MHz}, \mathrm{CDCl}_{3}$

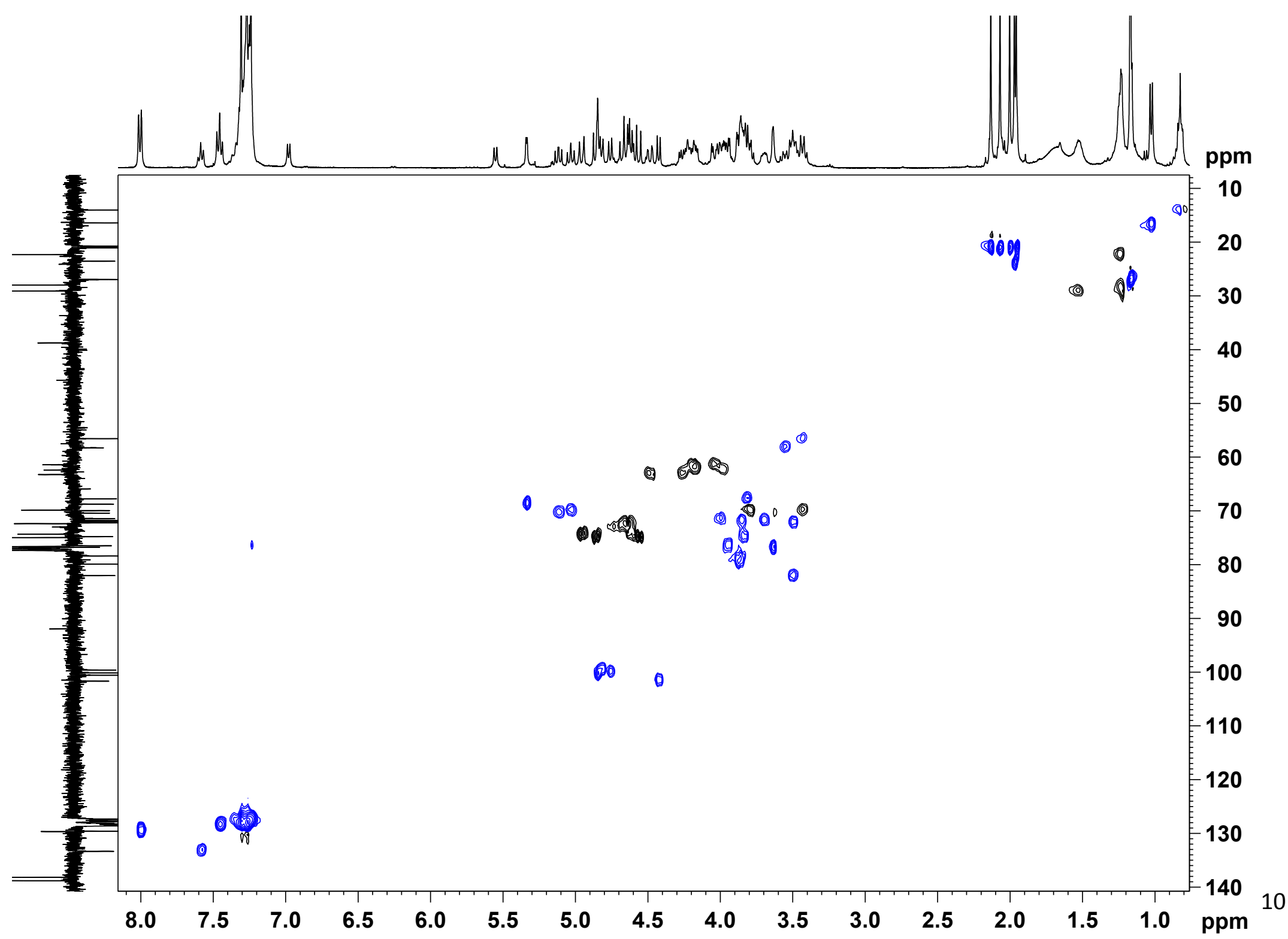


Compound 11, $400 \mathrm{MHz}, \mathrm{CDCl}_{3}$
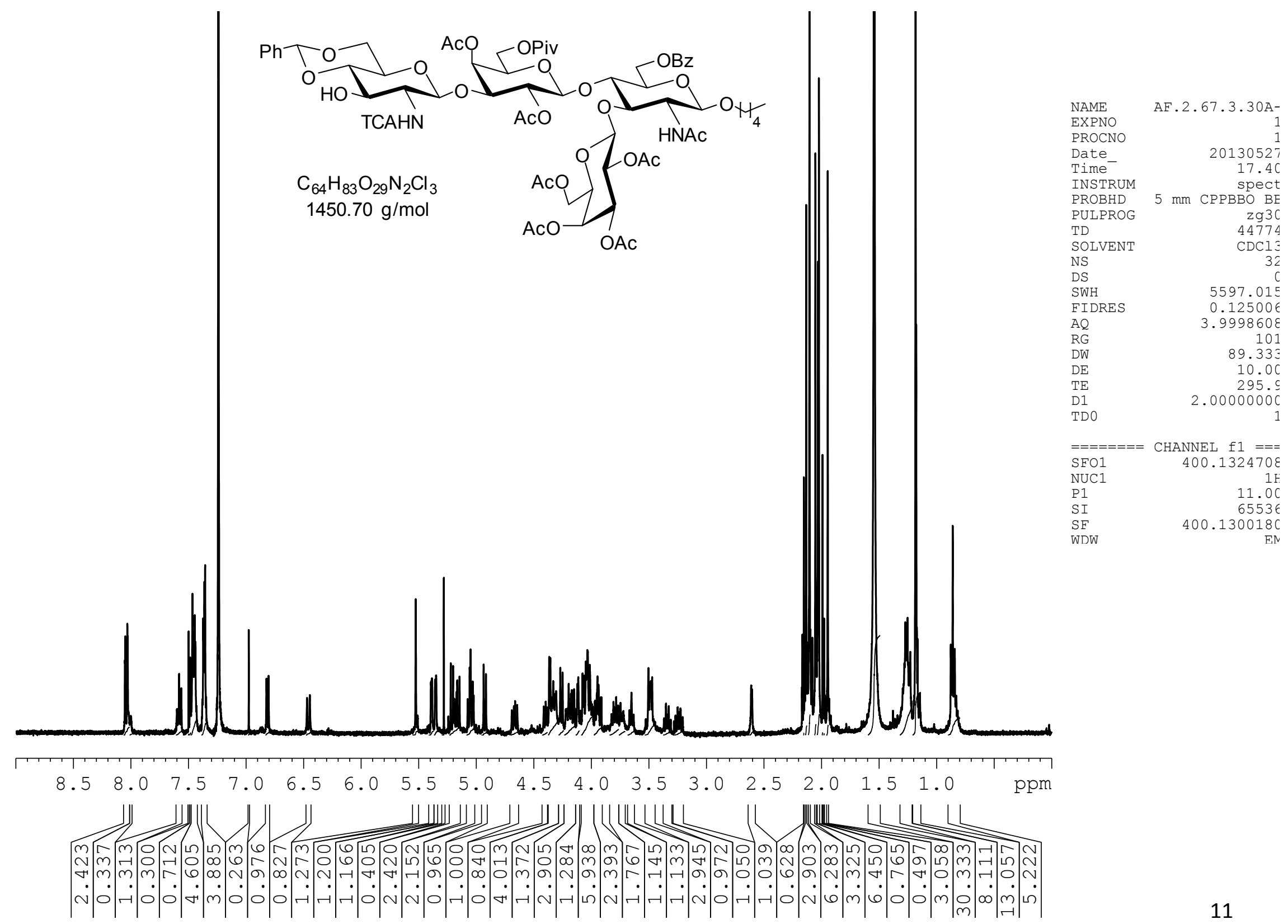

11 
Compound 11, $400 \mathrm{MHz}, \mathrm{CDCl}_{3}$

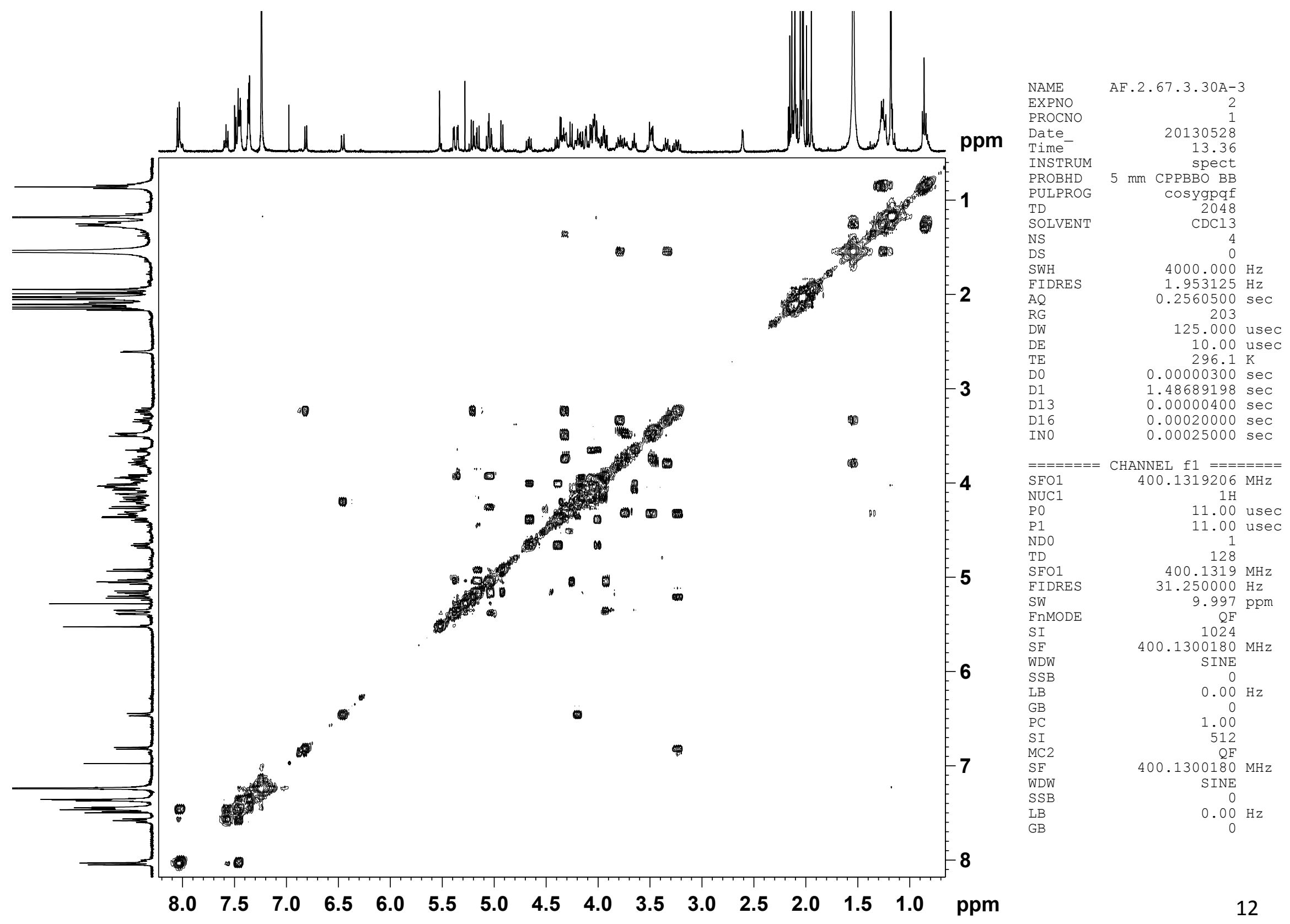


Compound 11, $101 \mathrm{MHz}, \mathrm{CDCl}_{3}$

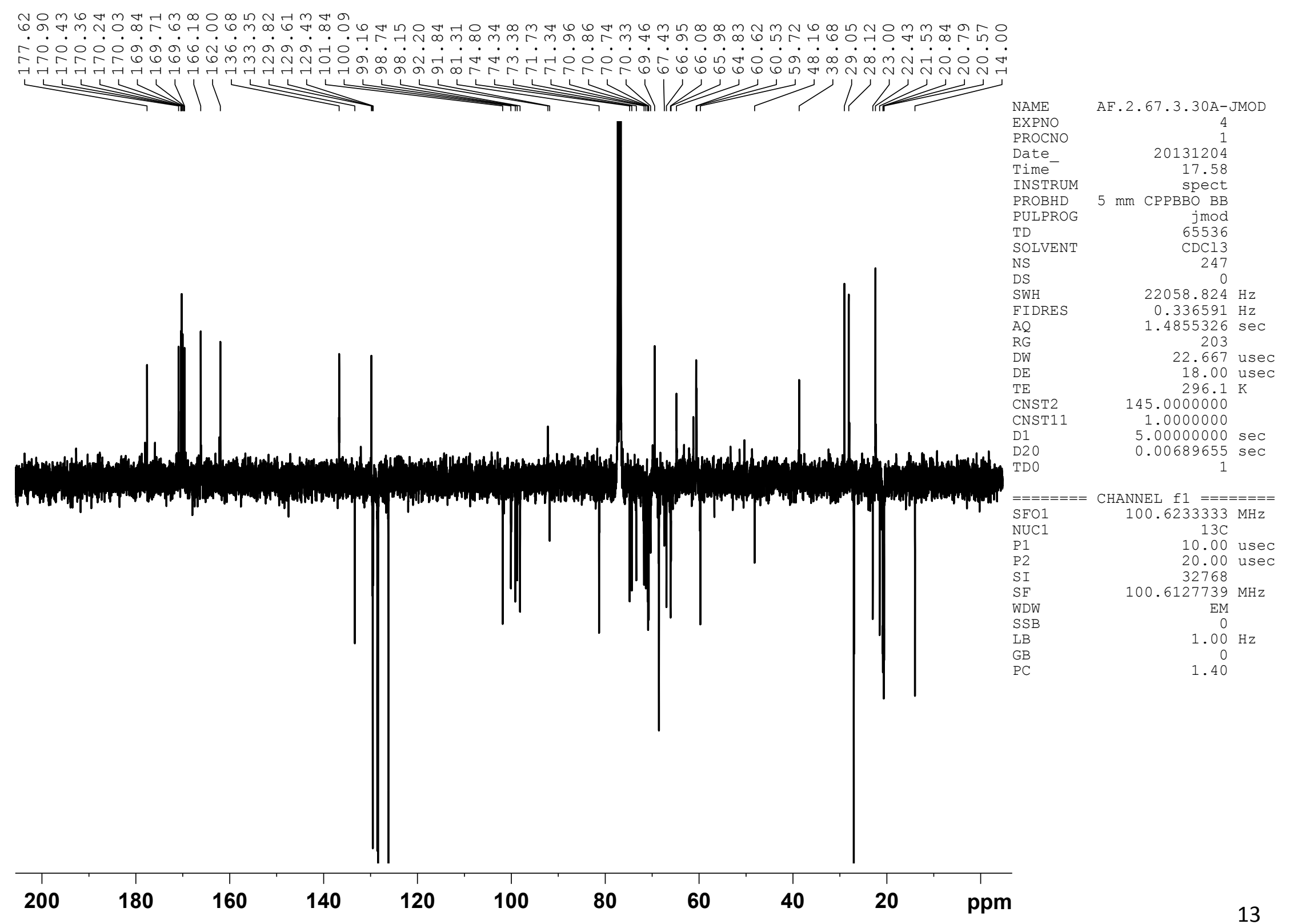


Compound 11, HSQC, $400 \mathrm{MHz}, \mathrm{CDCl}_{3}$

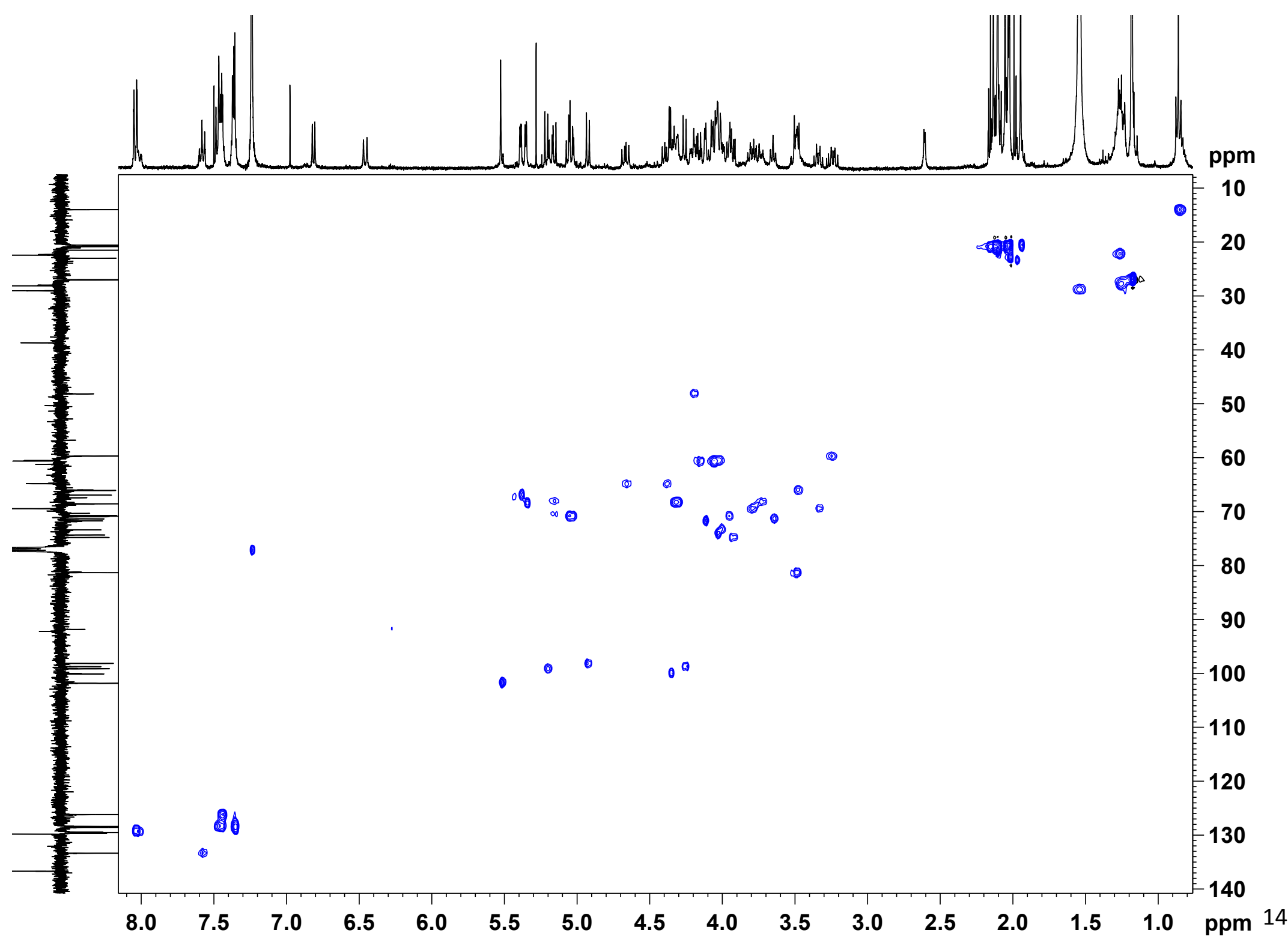


Compound 12, $400 \mathrm{MHz}, \mathrm{CDCl}_{3}$

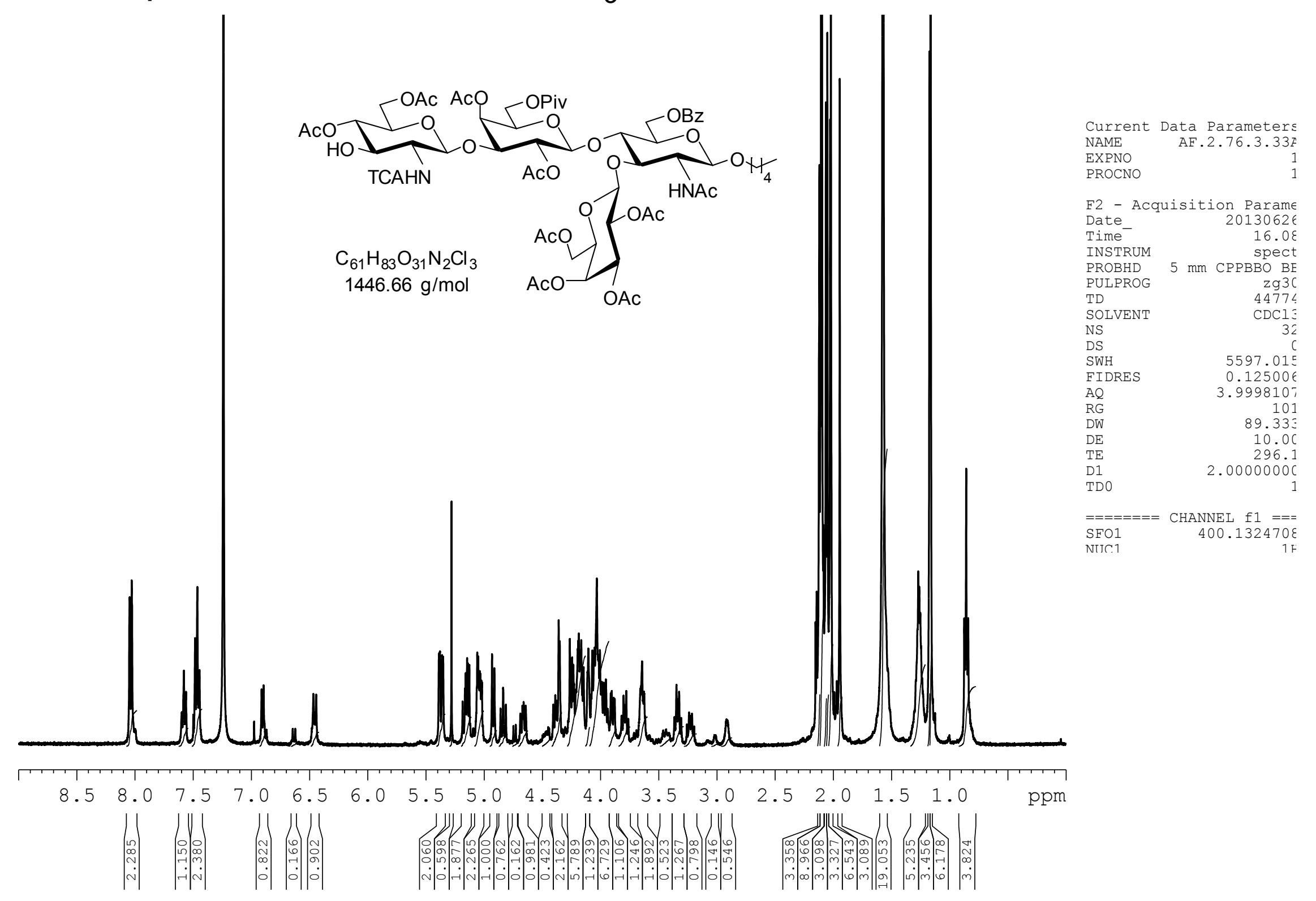


Compound 12, COSY, $400 \mathrm{MHz}, \mathrm{CDCl}_{3}$

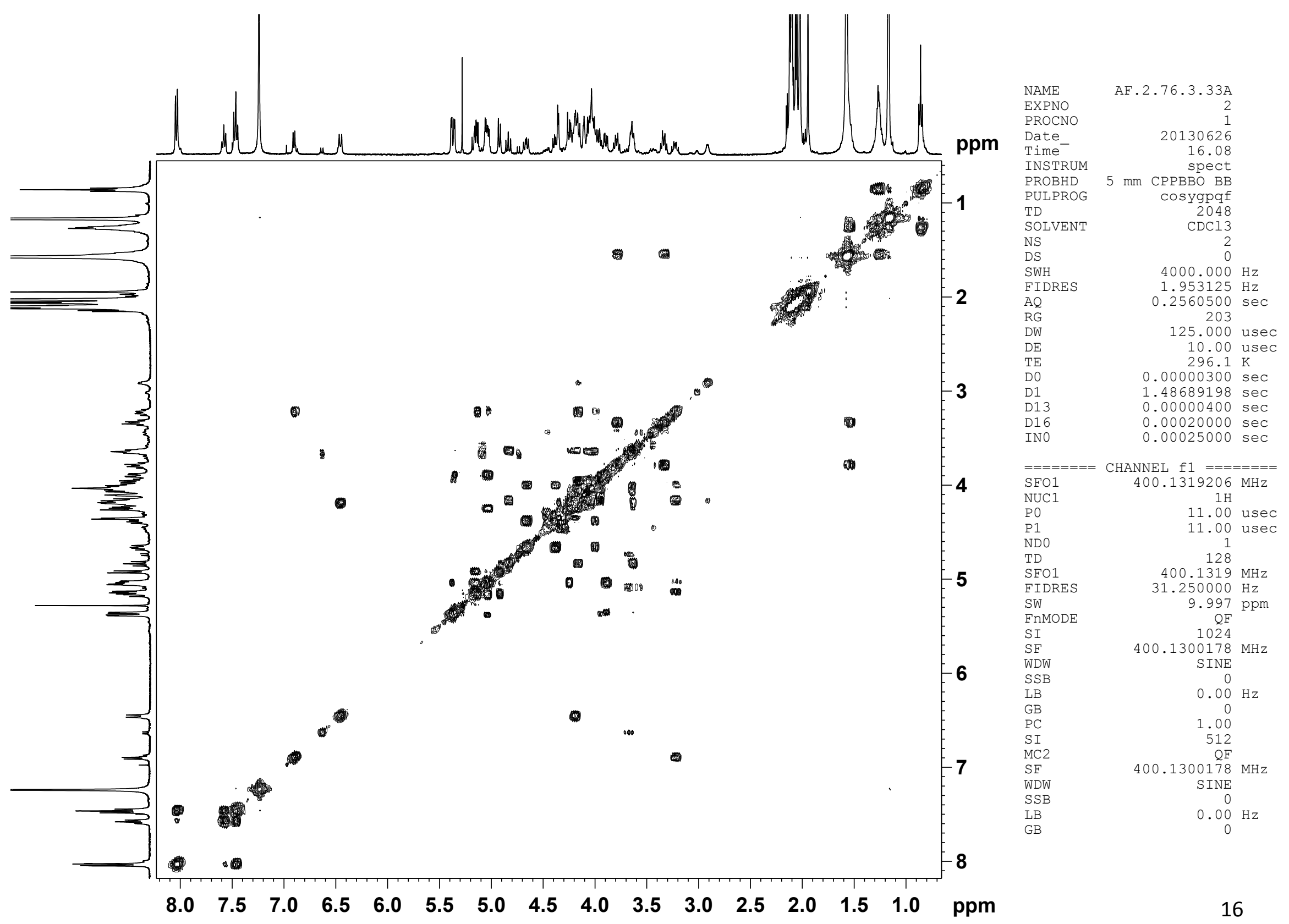


Compound $12 \sim 80 \%$ pure (see text, $20 \%$ suspected orthoester), $101 \mathrm{MHz}$, $\mathrm{CDCl}_{3}$

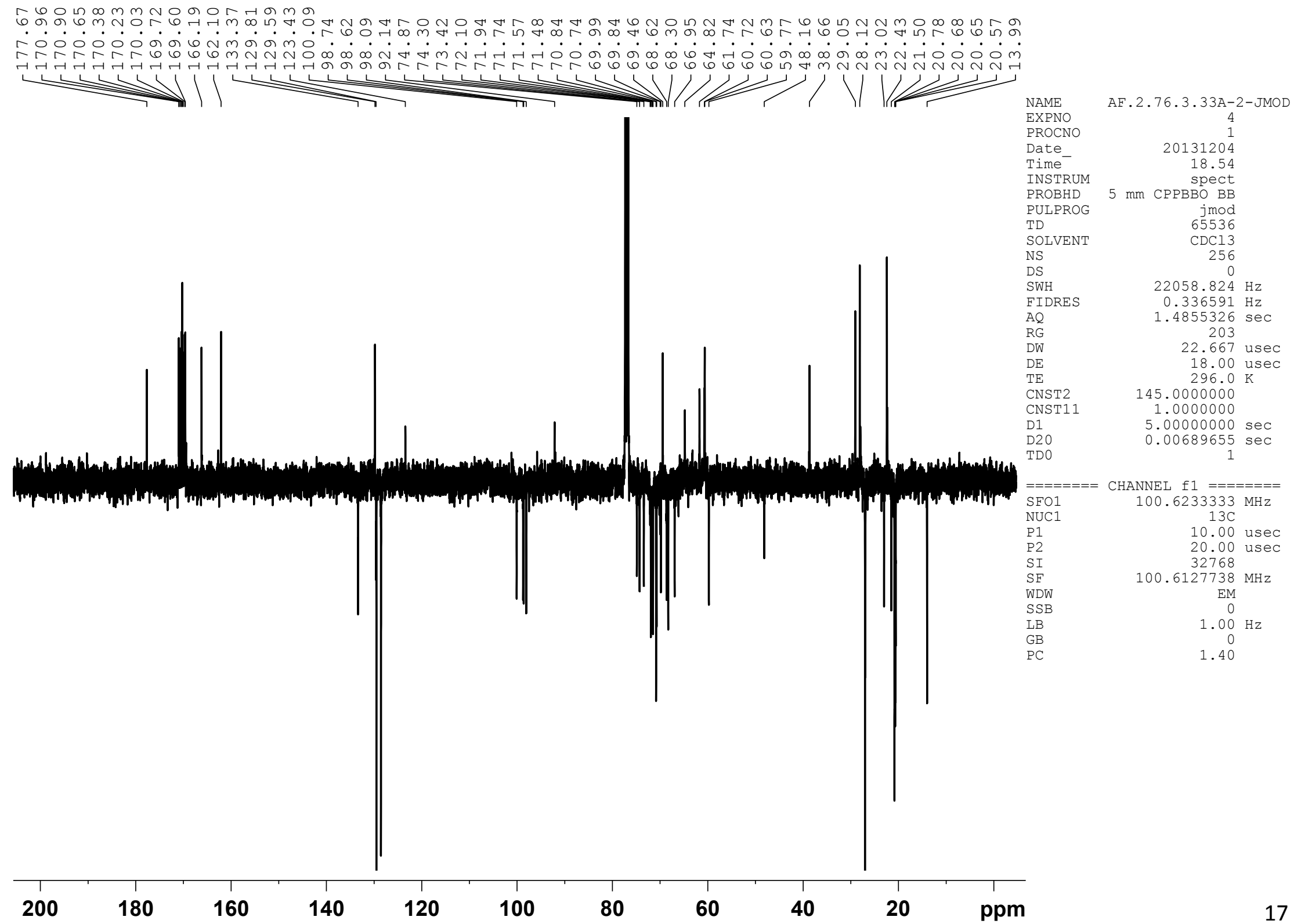


Compound 12, edited HSQC ( $\mathrm{CH}$ and $\mathrm{CH}_{3}$ blue, $\mathrm{CH}_{2}$ black), $400 \mathrm{MHz}, \mathrm{CDCl}_{3}$

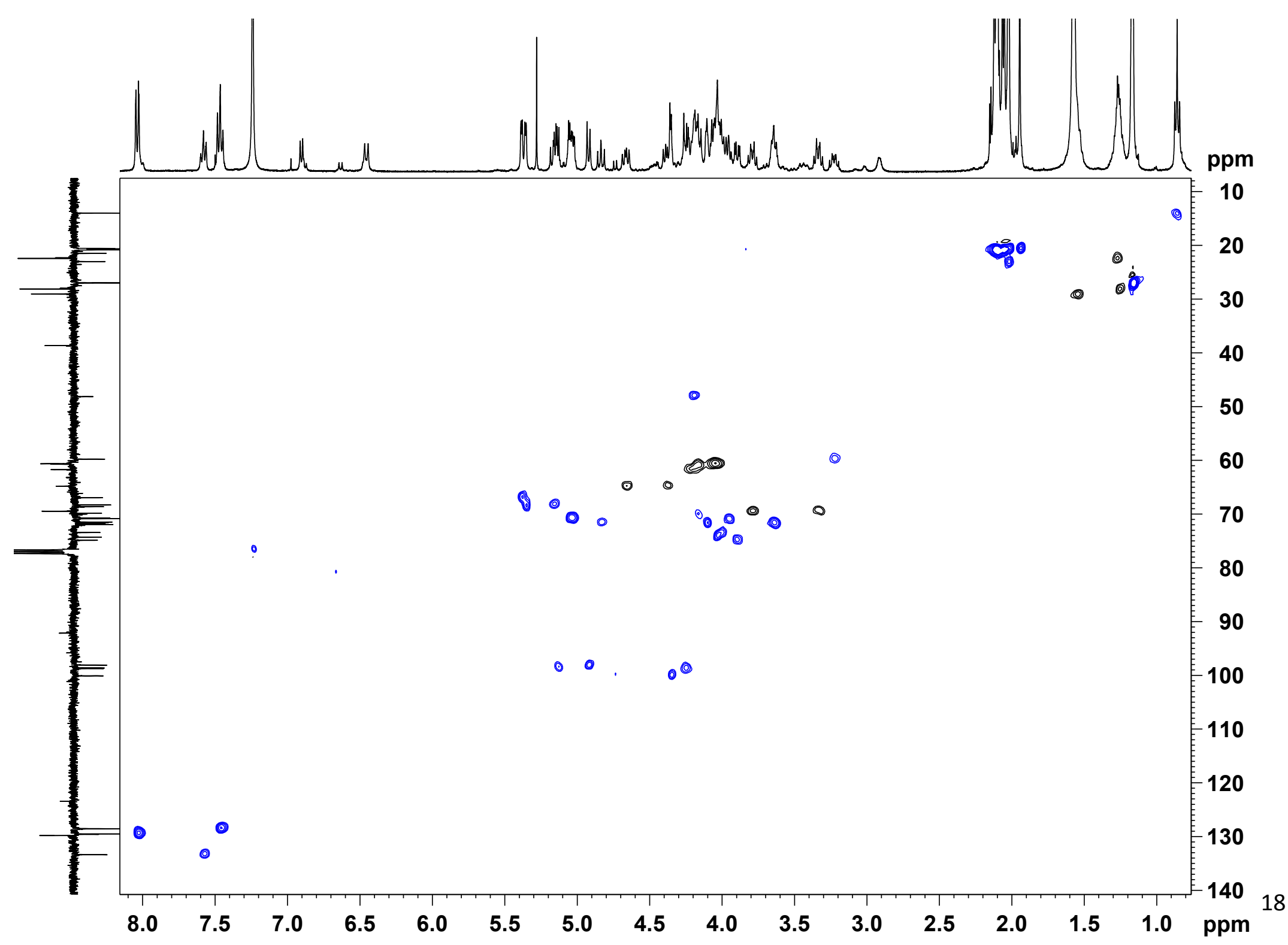




\section{Compound 13, $400 \mathrm{MHz}, \mathrm{CDCl}_{3}$}
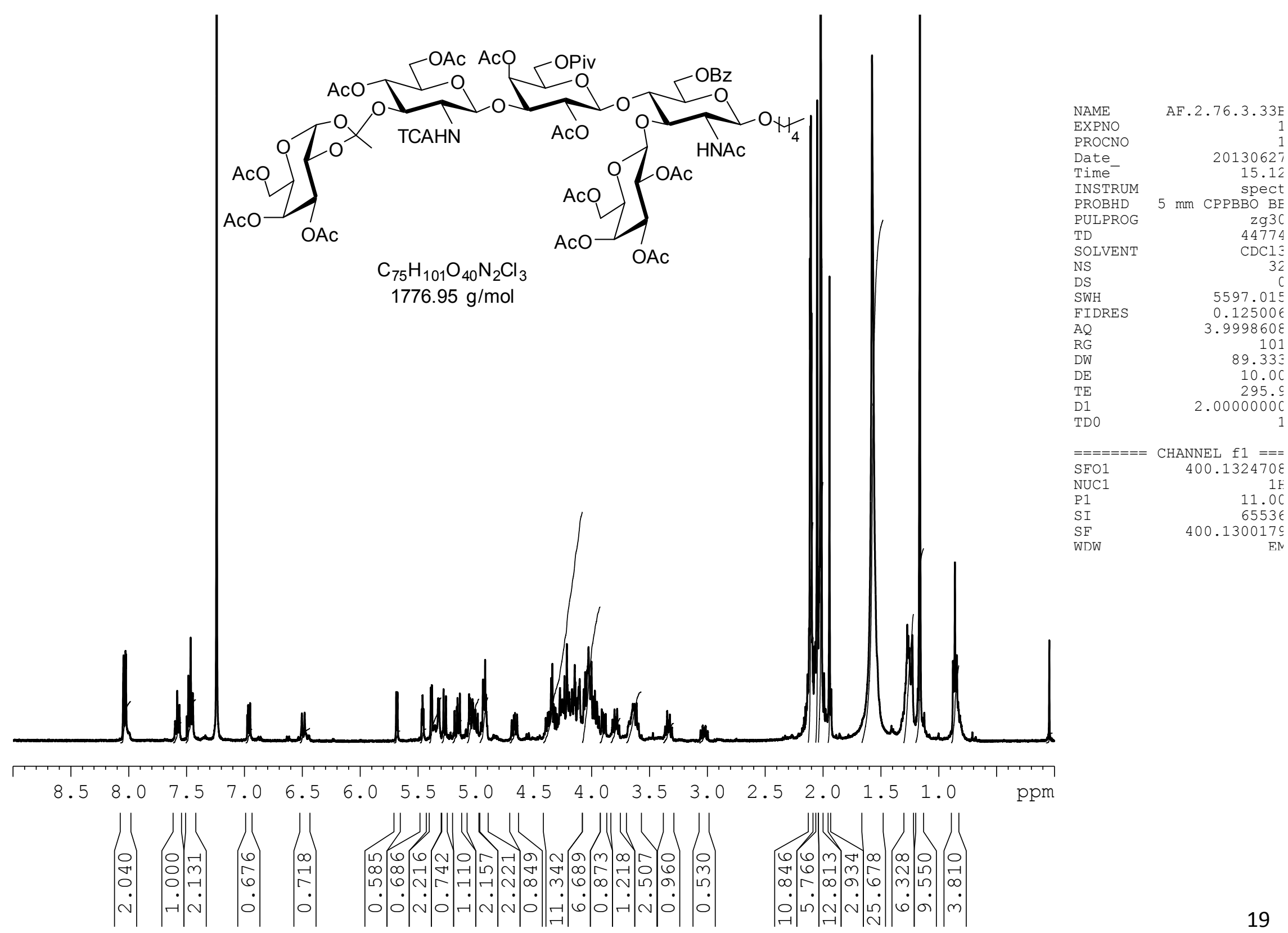
Compound 13, COSY, $400 \mathrm{MHz}, \mathrm{CDCl}_{3}$

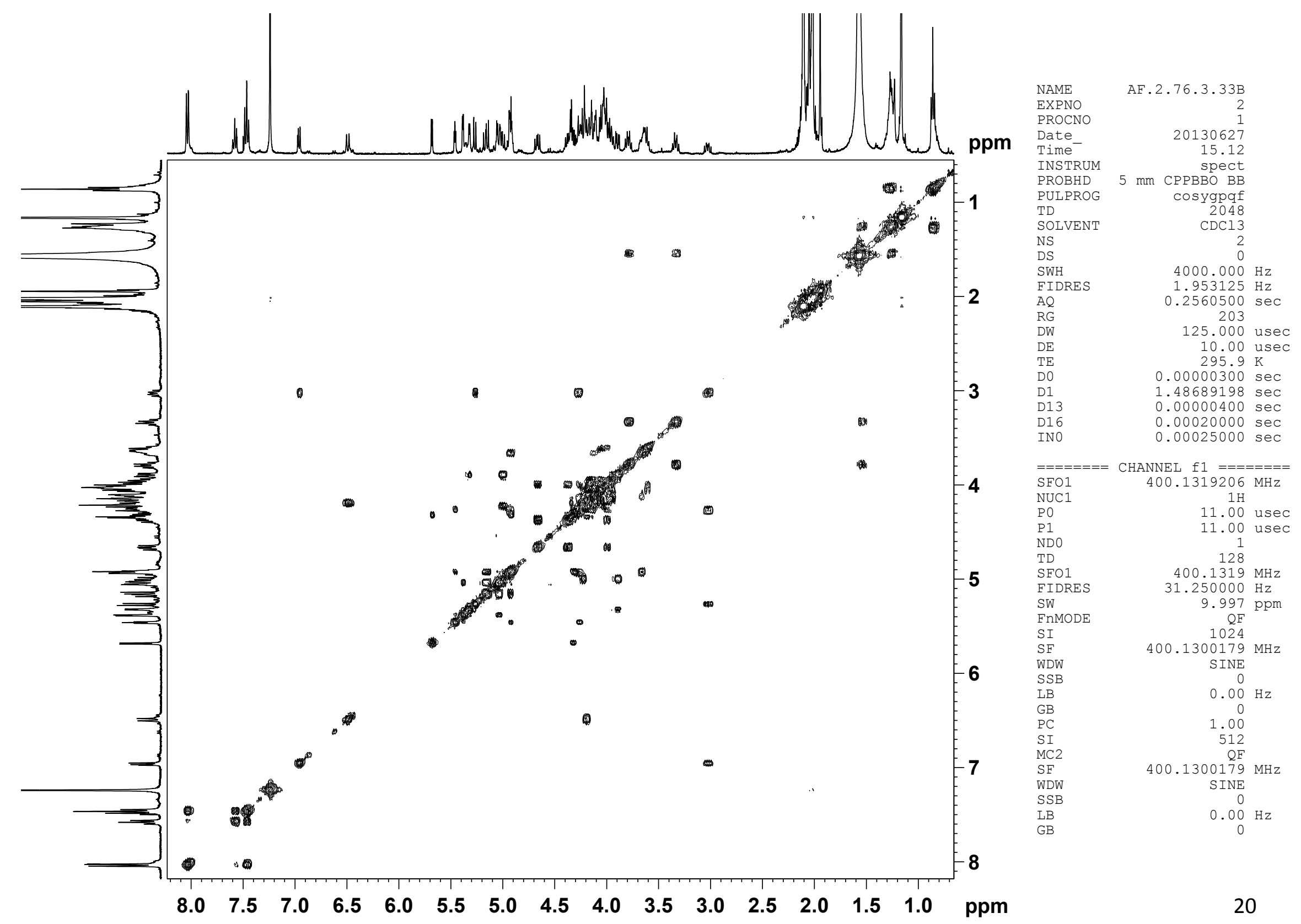




\section{Compound 13, $151 \mathrm{MHz}, \mathrm{CDCl}_{3}$}

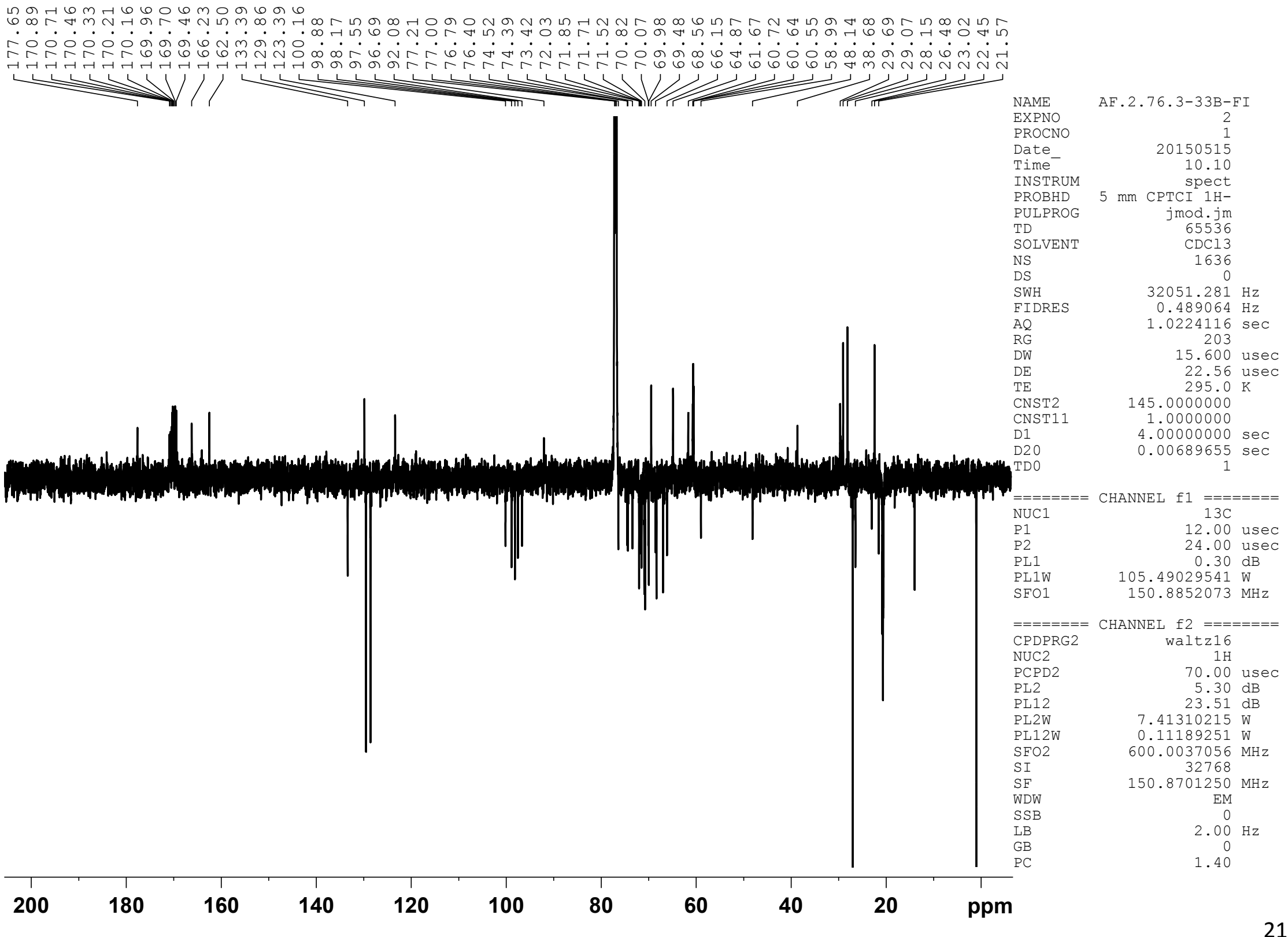


Compound 13, edited HSQC ( $\mathrm{CH}$ and $\mathrm{CH}_{3}$ blue, $\mathrm{CH}_{2}$ black), $400 \mathrm{MHz}, \mathrm{CDCl}_{3}$

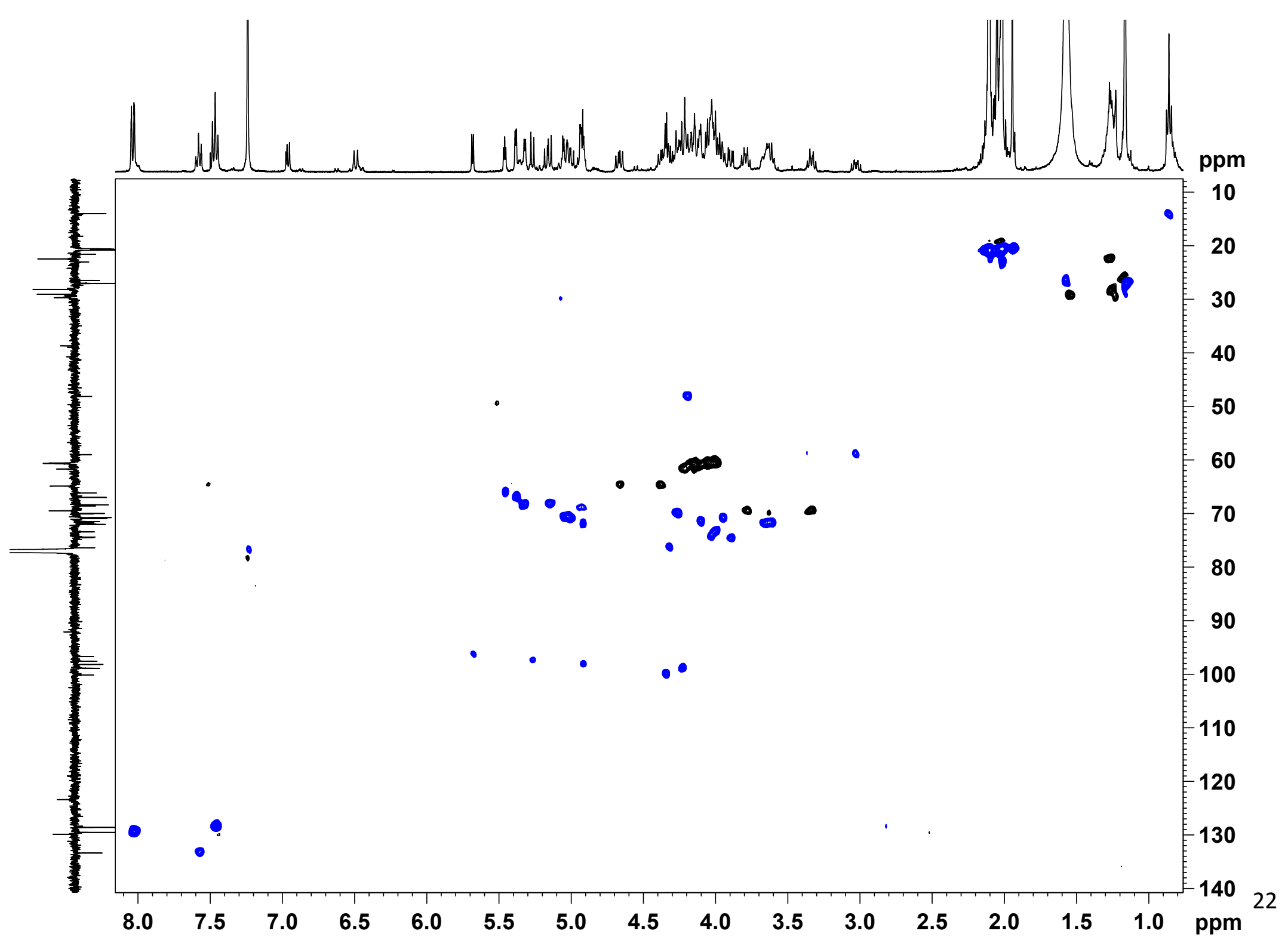


Compound 14, $400 \mathrm{MHz}, \mathrm{CDCl}_{3}$

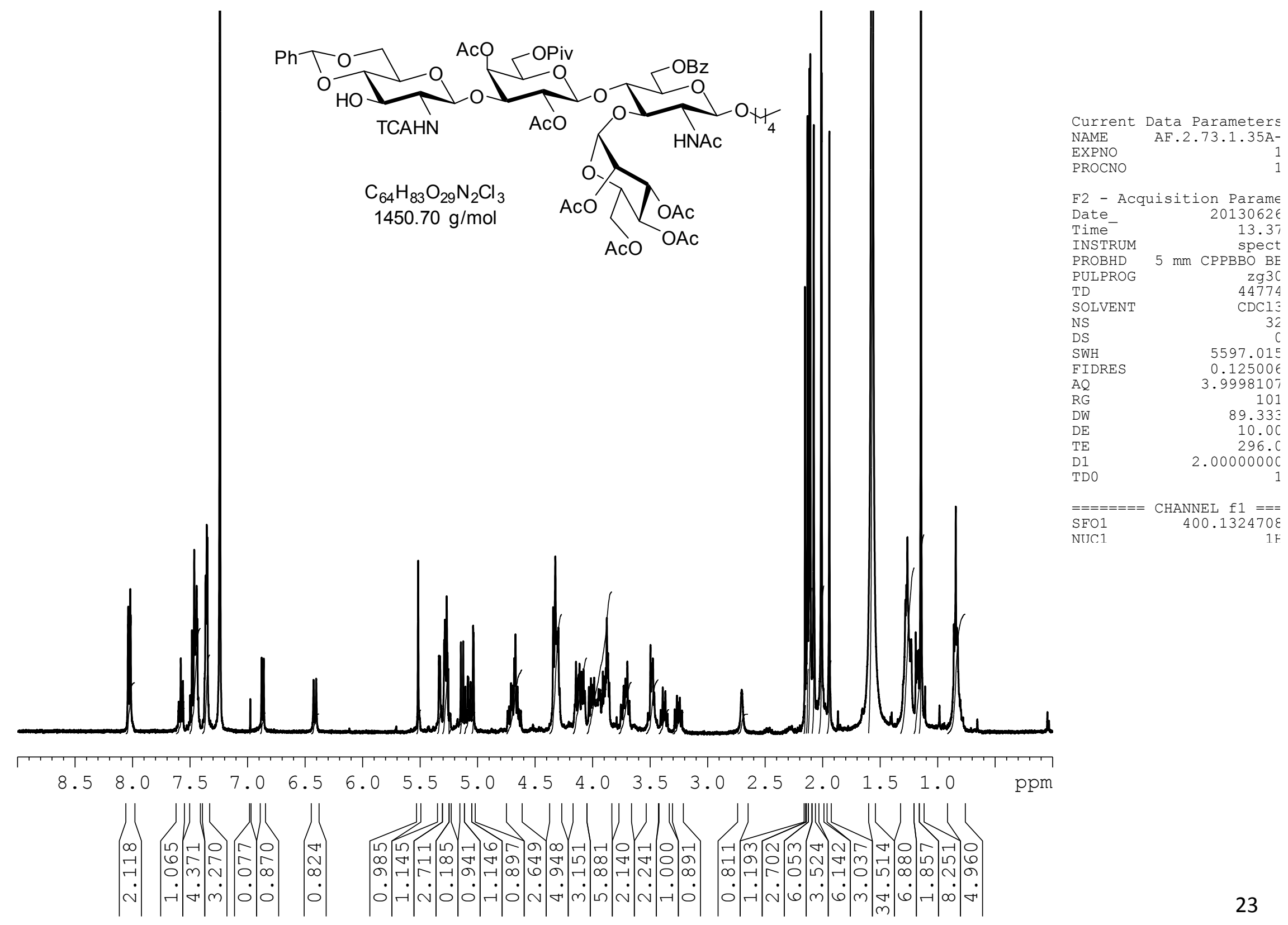


Compound 14, COSY, $400 \mathrm{MHz}, \mathrm{CDCl}_{3}$

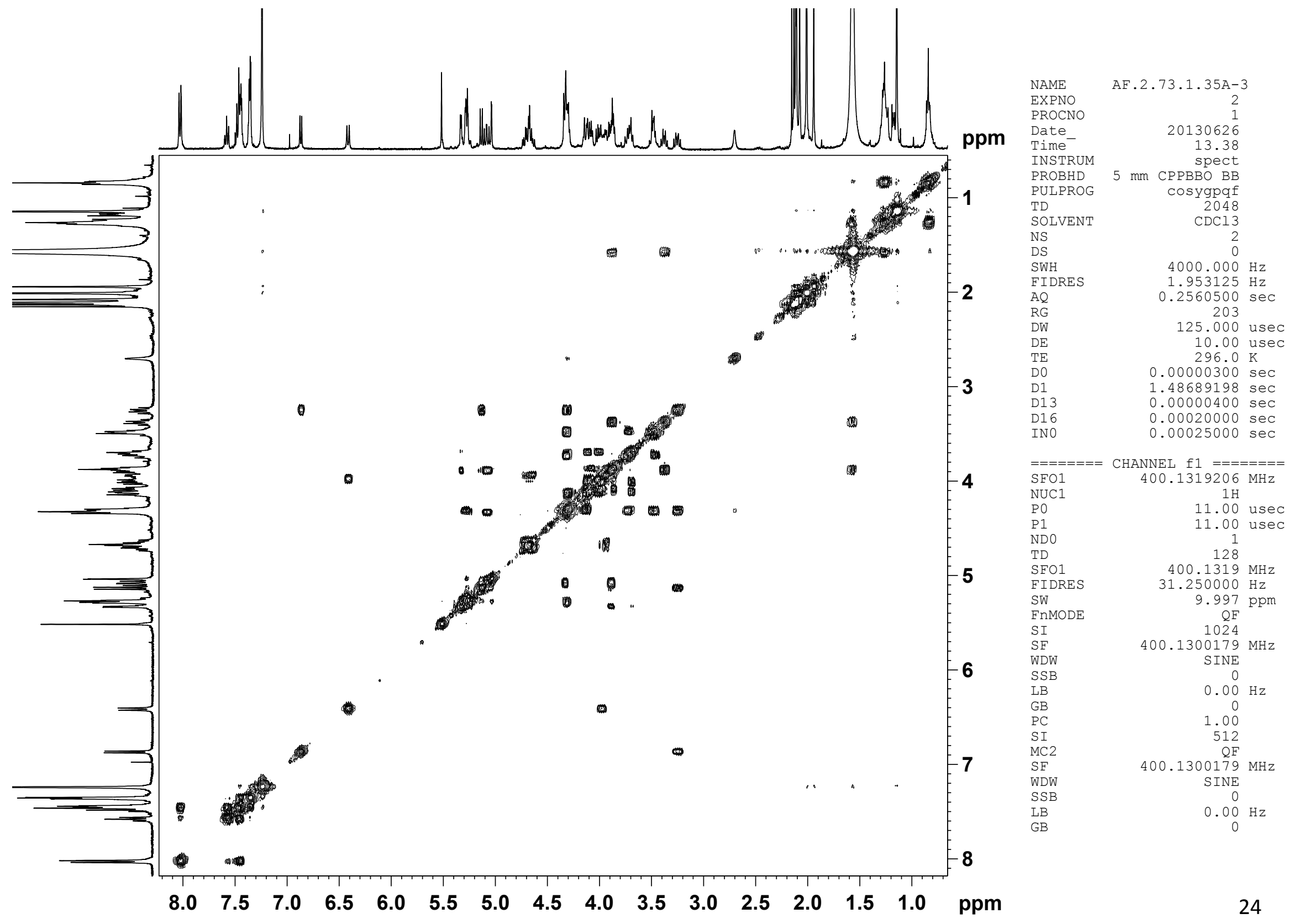


Compound 14, $101 \mathrm{MHz}, \mathrm{CDCl}_{3}$

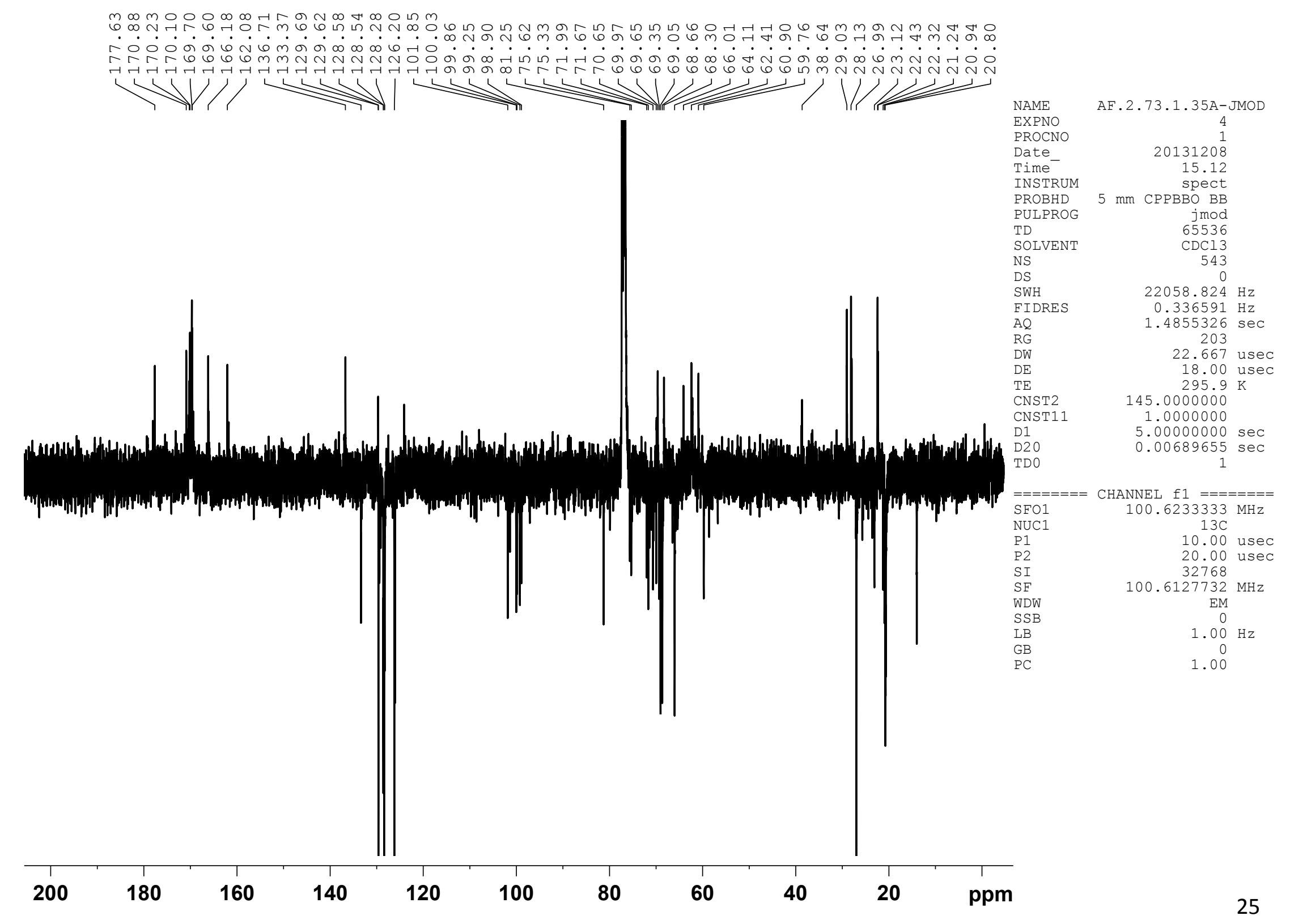


Compound 14, edited HSQC ( $\mathrm{CH}$ and $\mathrm{CH}_{3}$ blue, $\mathrm{CH}_{2}$ black) $400 \mathrm{MHz}, \mathrm{CDCl}_{3}$

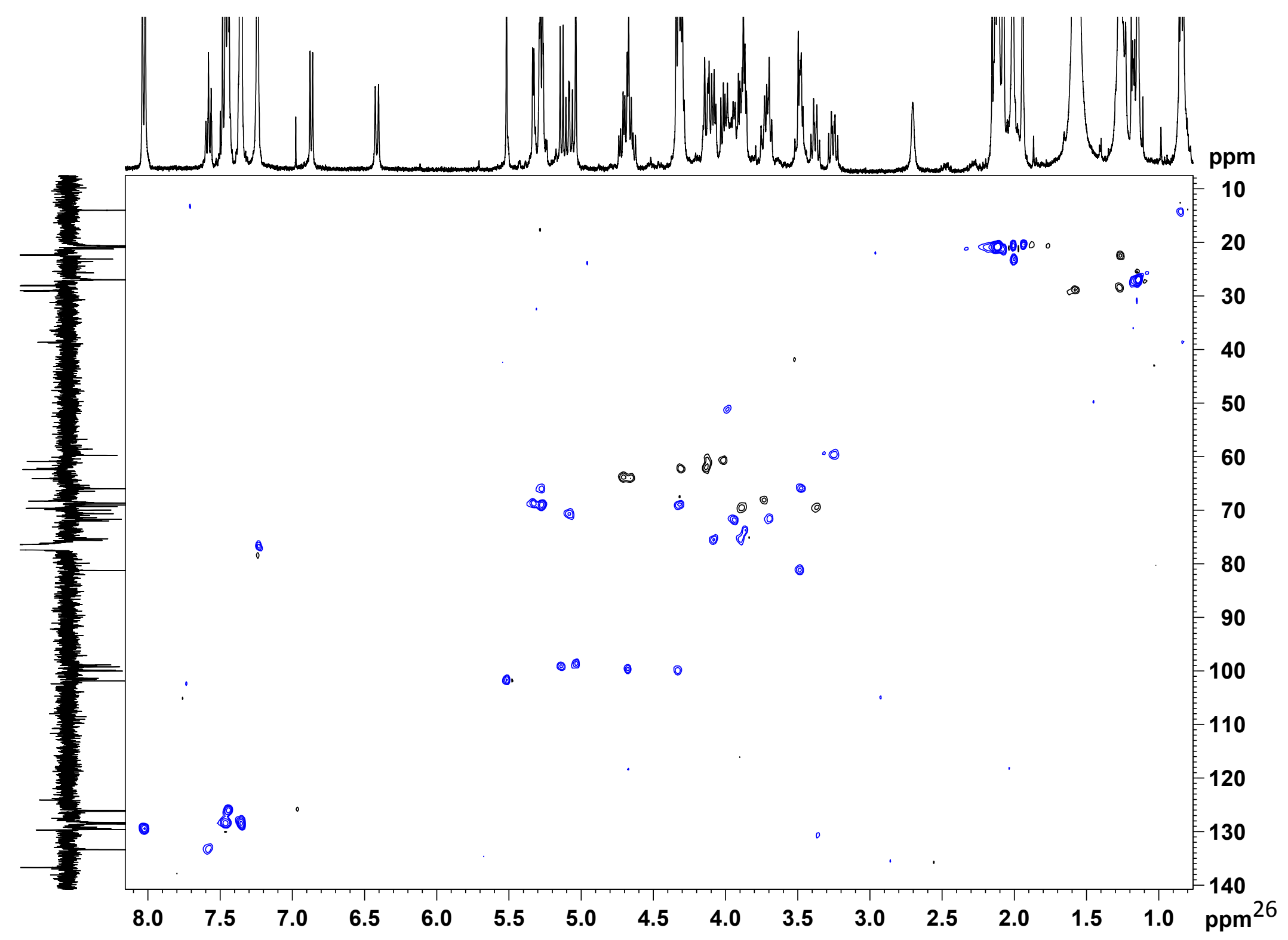


Compound 15, $400 \mathrm{MHz}, \mathrm{CDCl}_{3}$
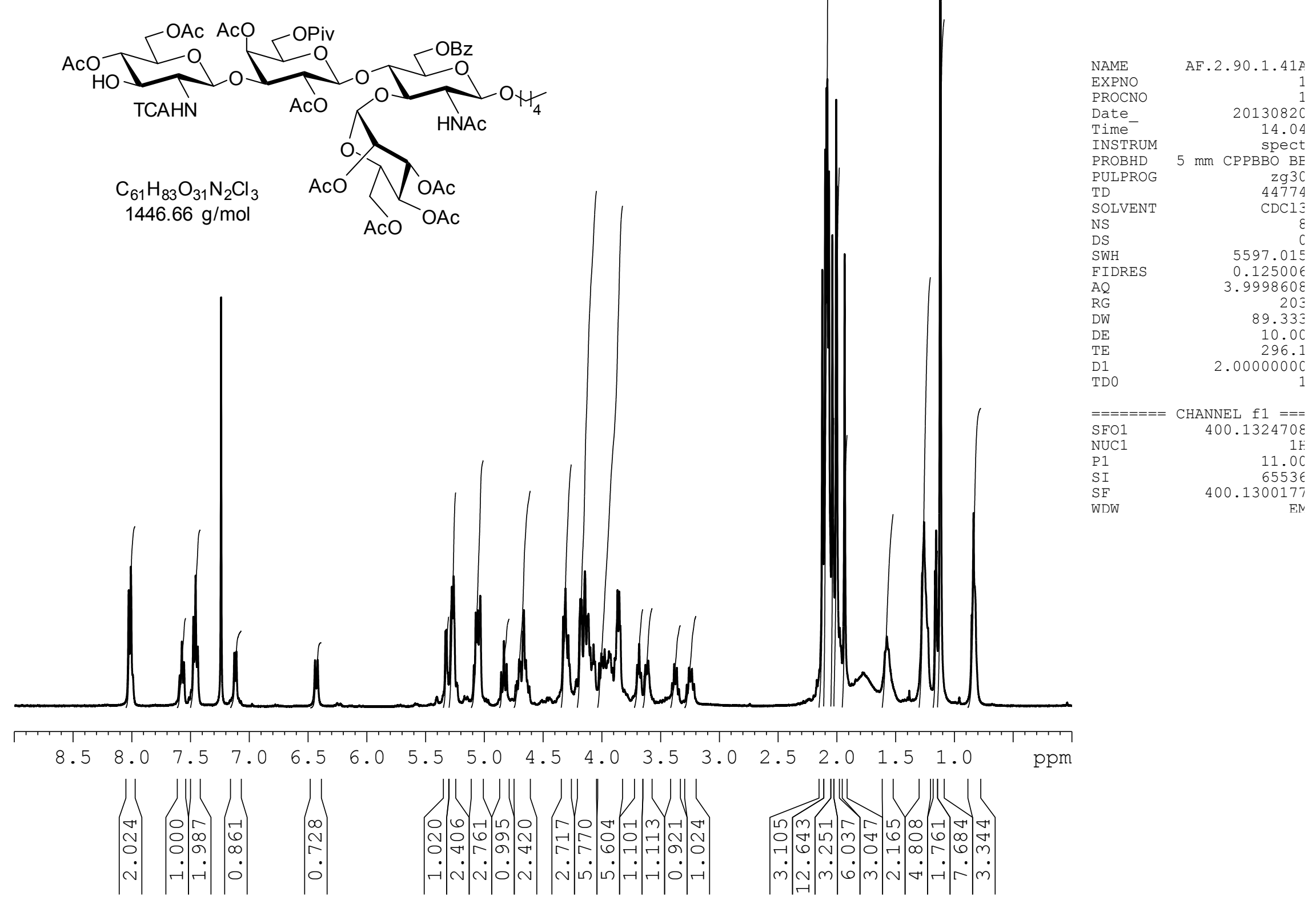
Compound 15, COSY, $400 \mathrm{MHz}, \mathrm{CDCl}_{3}$

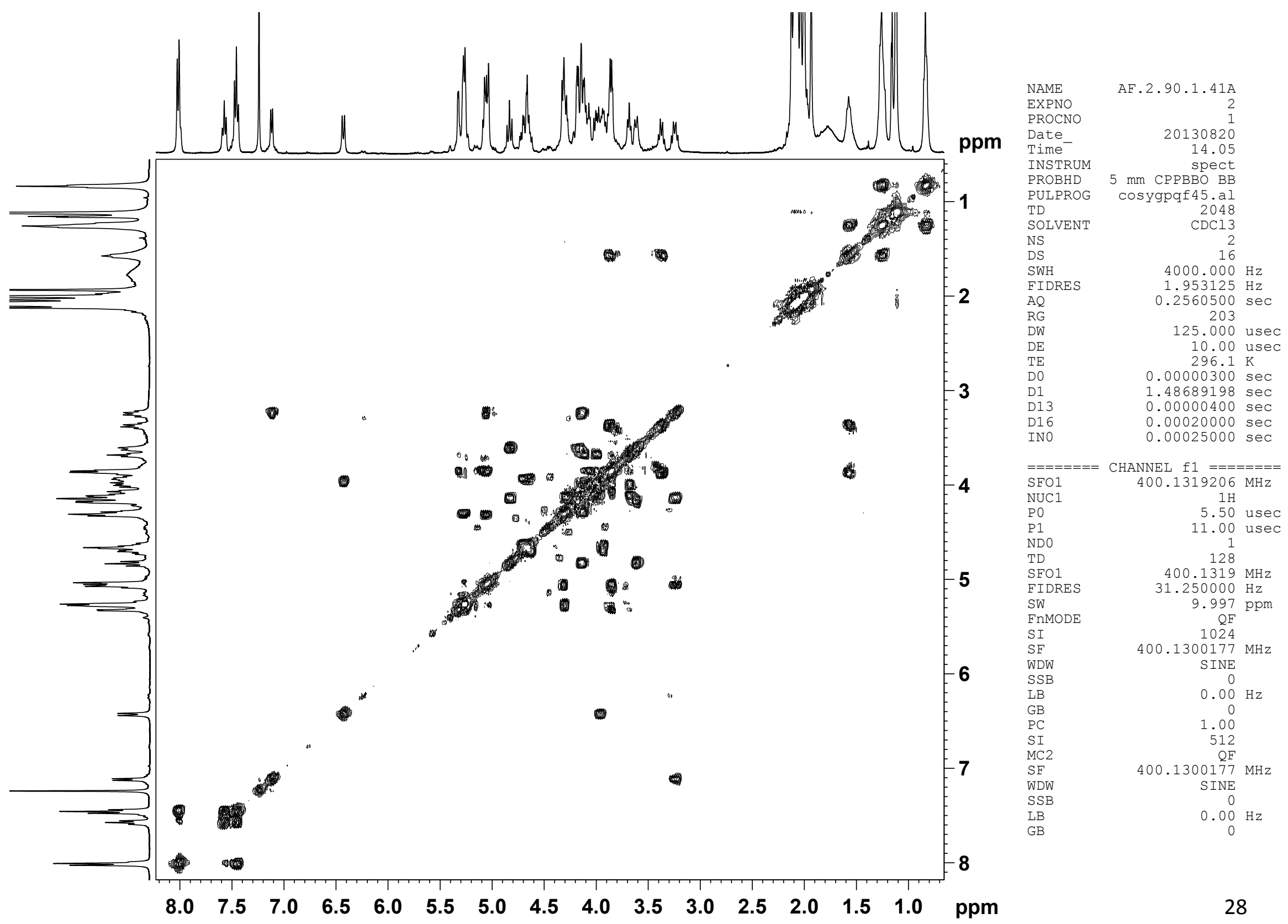




\section{Compound 15, $101 \mathrm{MHz}, \mathrm{CDCl}_{3}$}

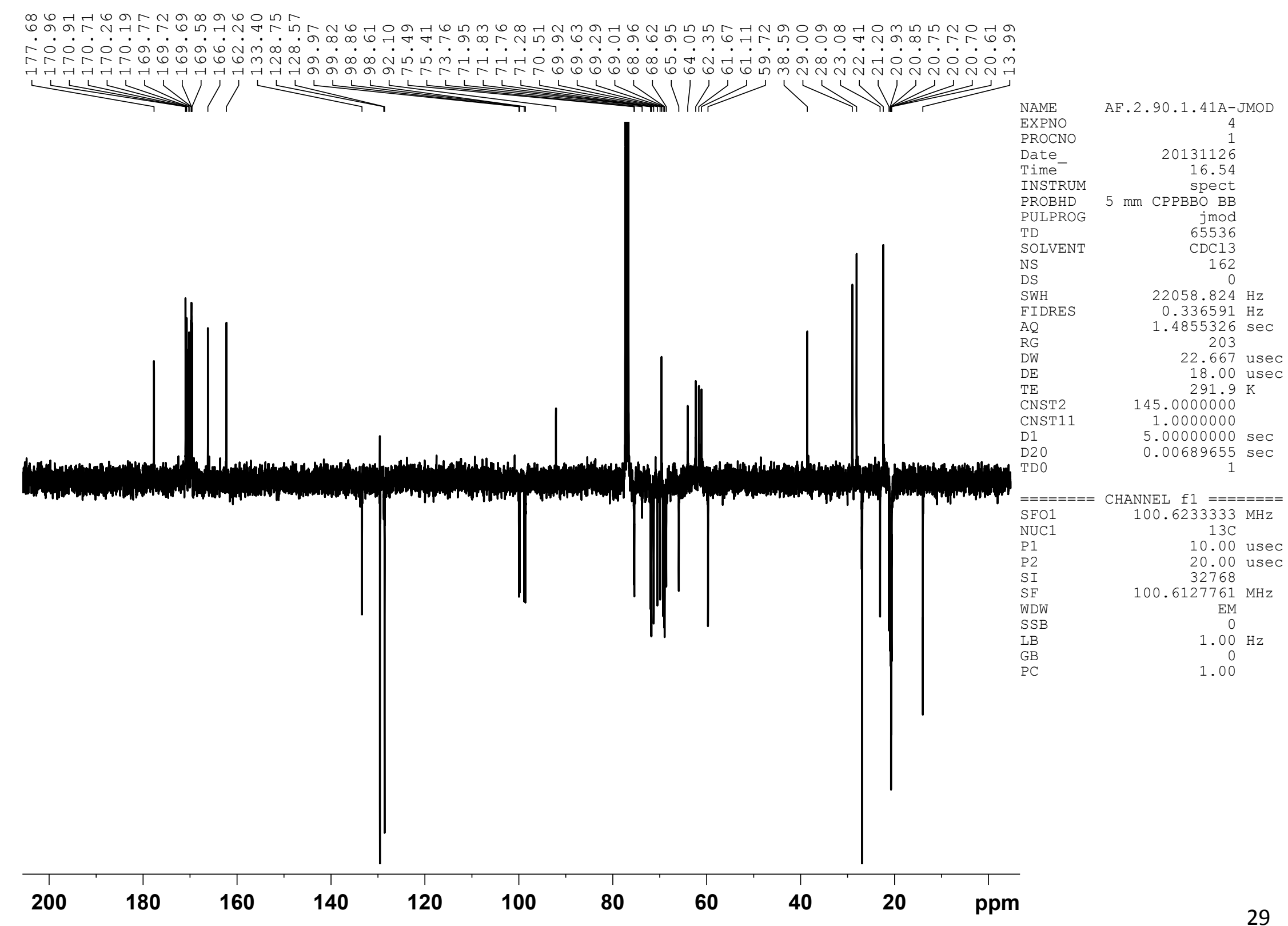


Compound 15, edited $\mathrm{HSQC}\left(\mathrm{CH}\right.$ and $\mathrm{CH}_{3}$ blue, $\mathrm{CH}_{2}$ black), $400 \mathrm{MHz}, \mathrm{CDCl}_{3}$

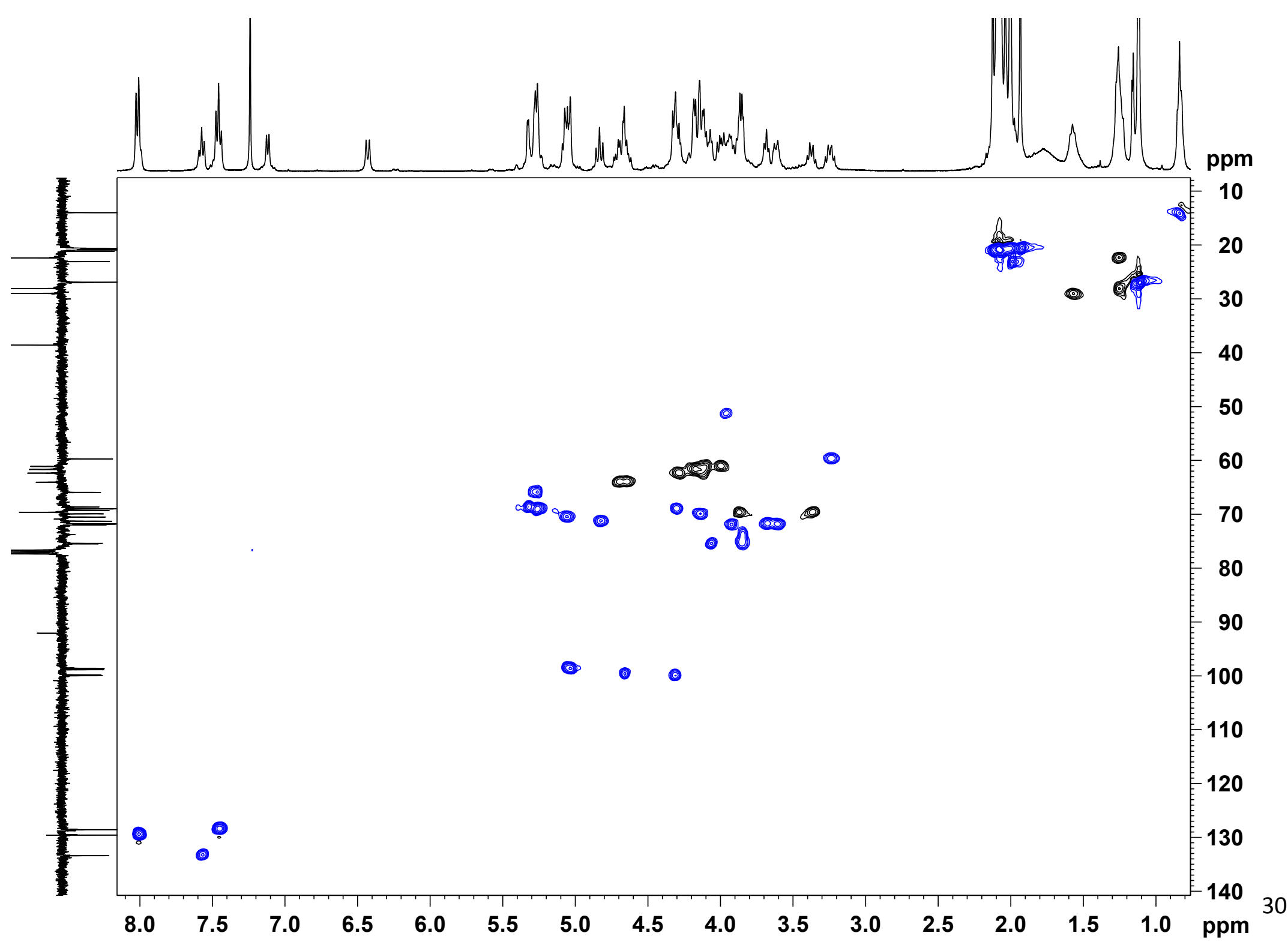


Compound 16, $400 \mathrm{MHz}, \mathrm{CDCl}_{3}$

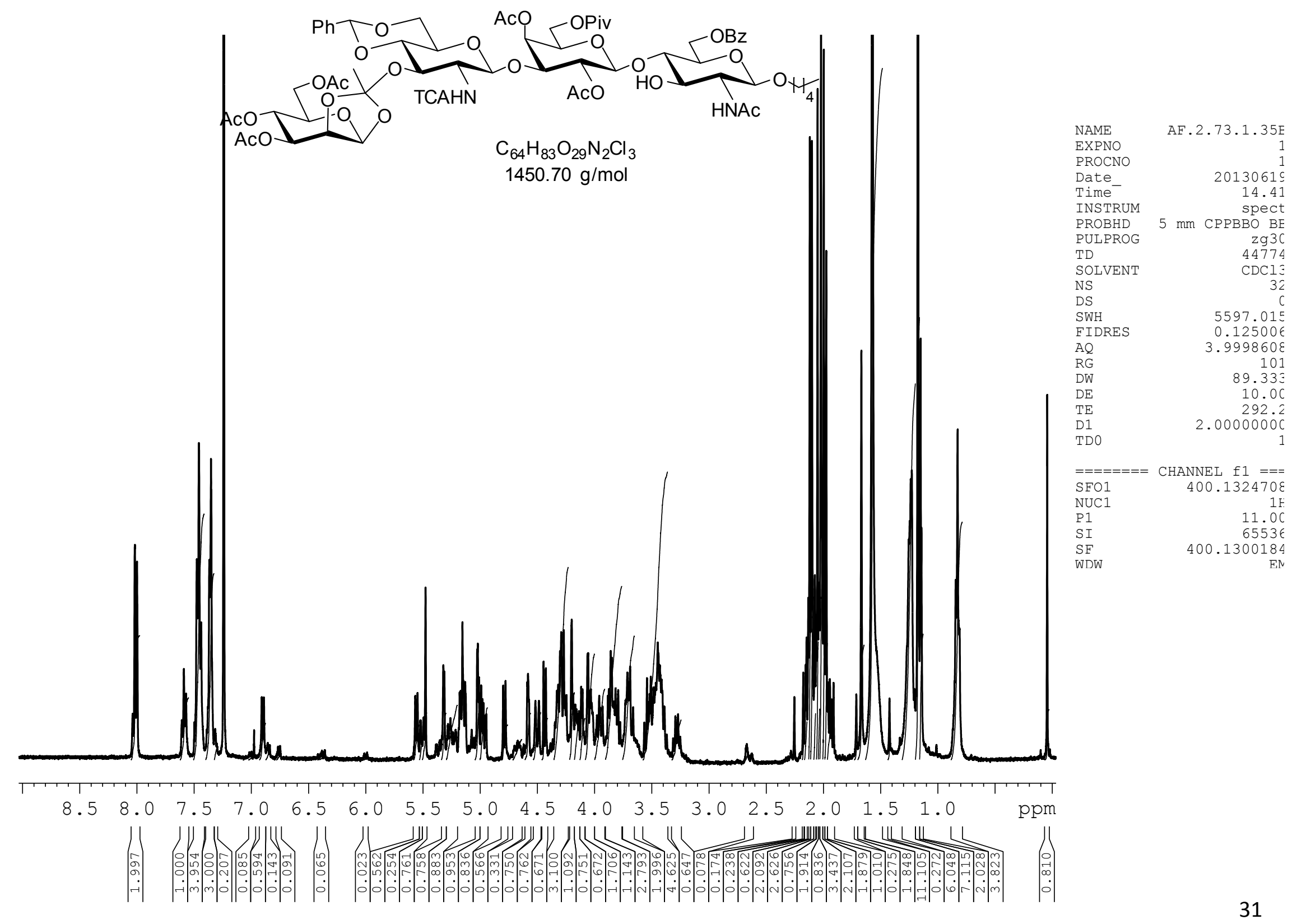


Compound 16, COSY, $400 \mathrm{MHz}, \mathrm{CDCl}_{3}$

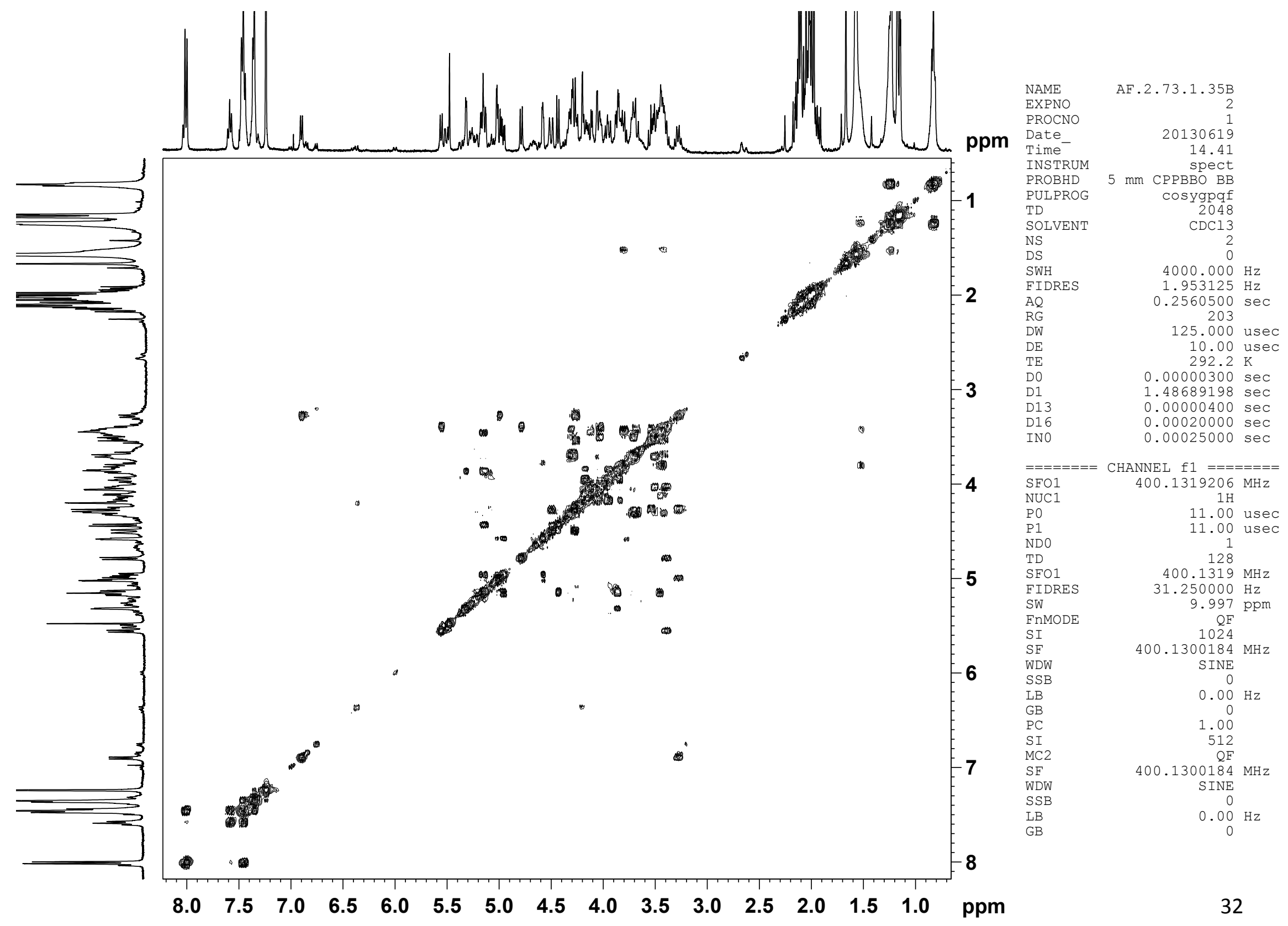


Compound 16, $101 \mathrm{MHz}, \mathrm{CDCl}_{3}$

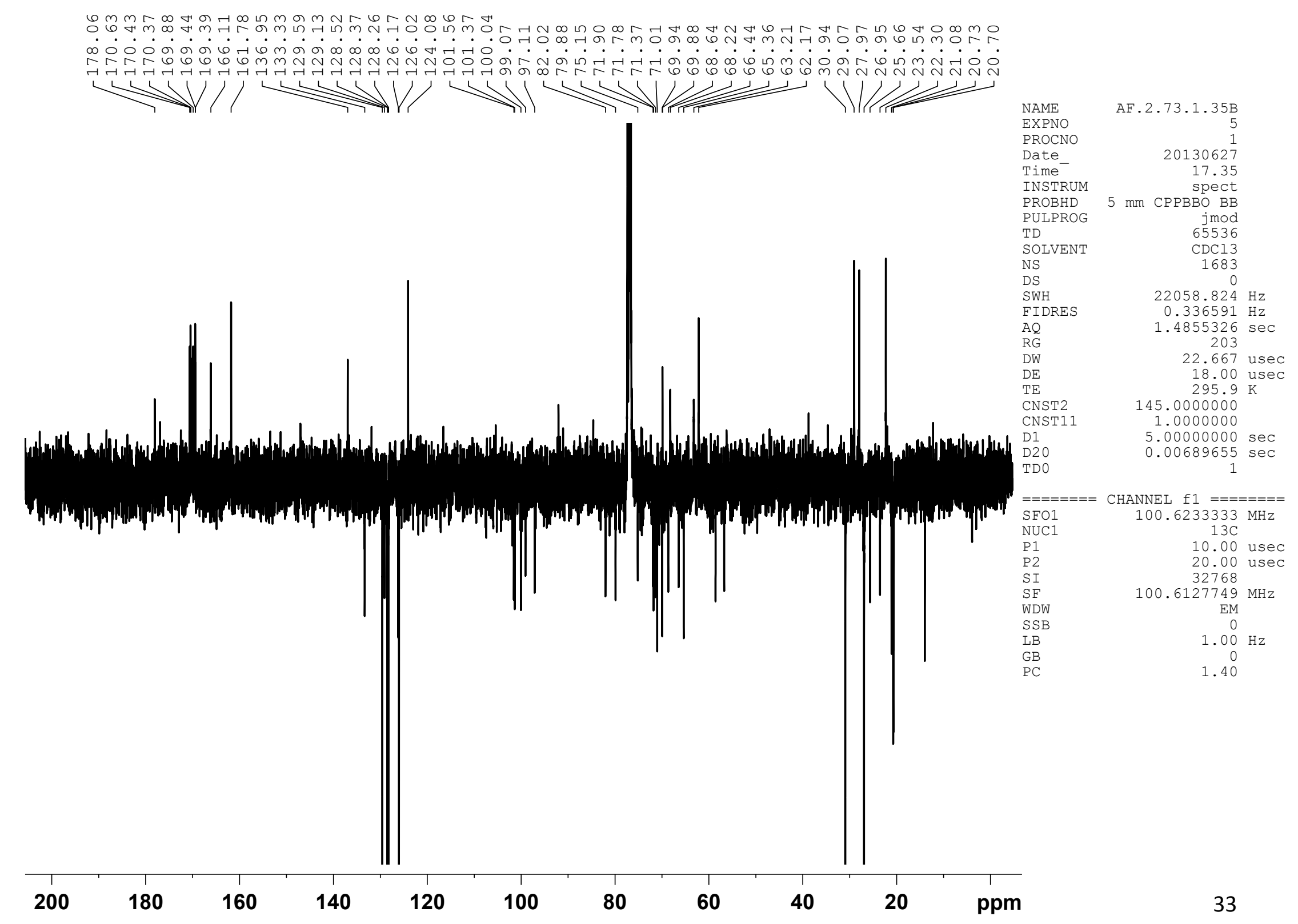




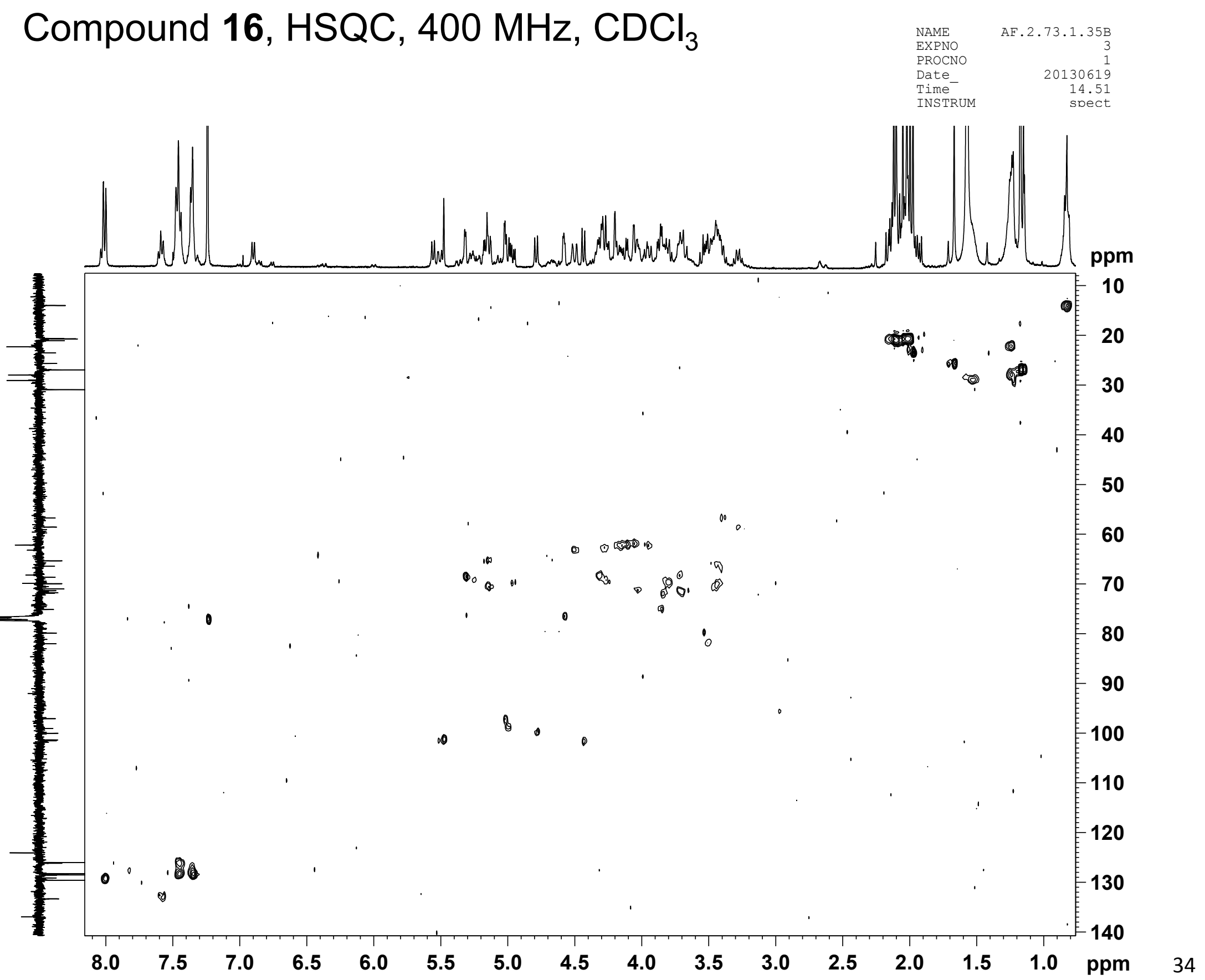


Compound 17, $400 \mathrm{MHz}, \mathrm{CDCl}_{3}$

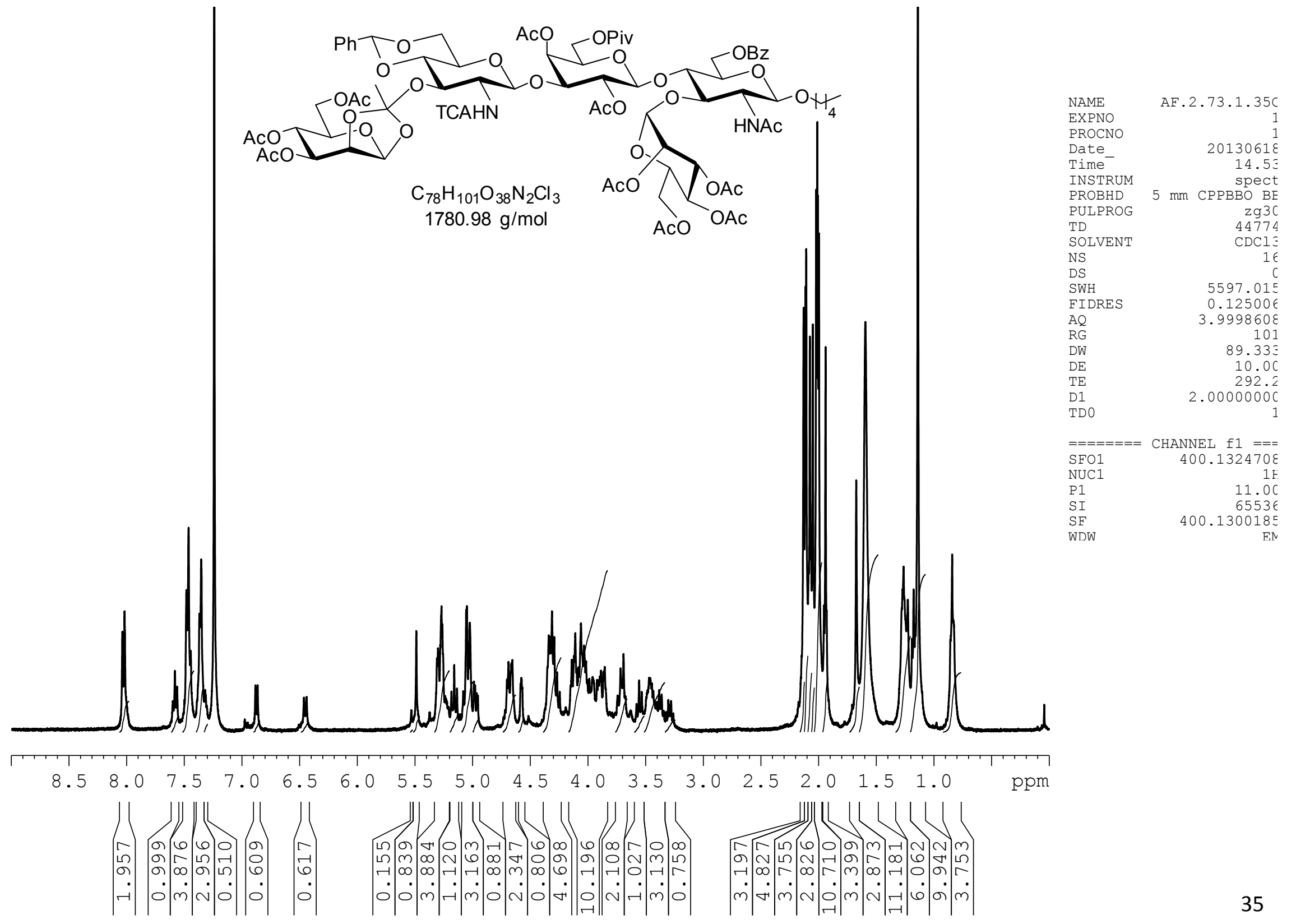


Compound 17, COSY, $400 \mathrm{MHz}, \mathrm{CDCl}_{3}$

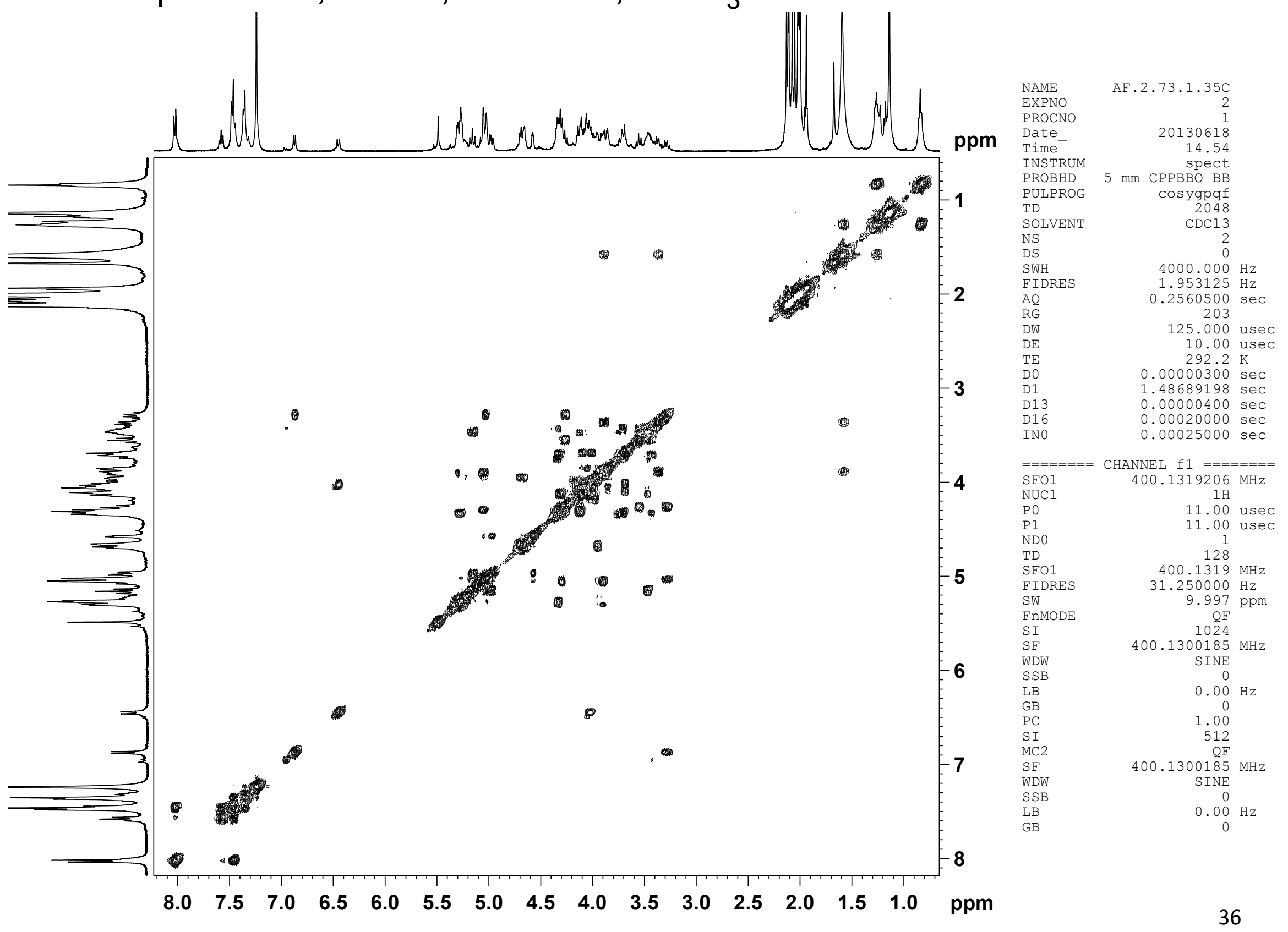




\section{Compound 17, $101 \mathrm{MHz}, \mathrm{CDCl}_{3}$}

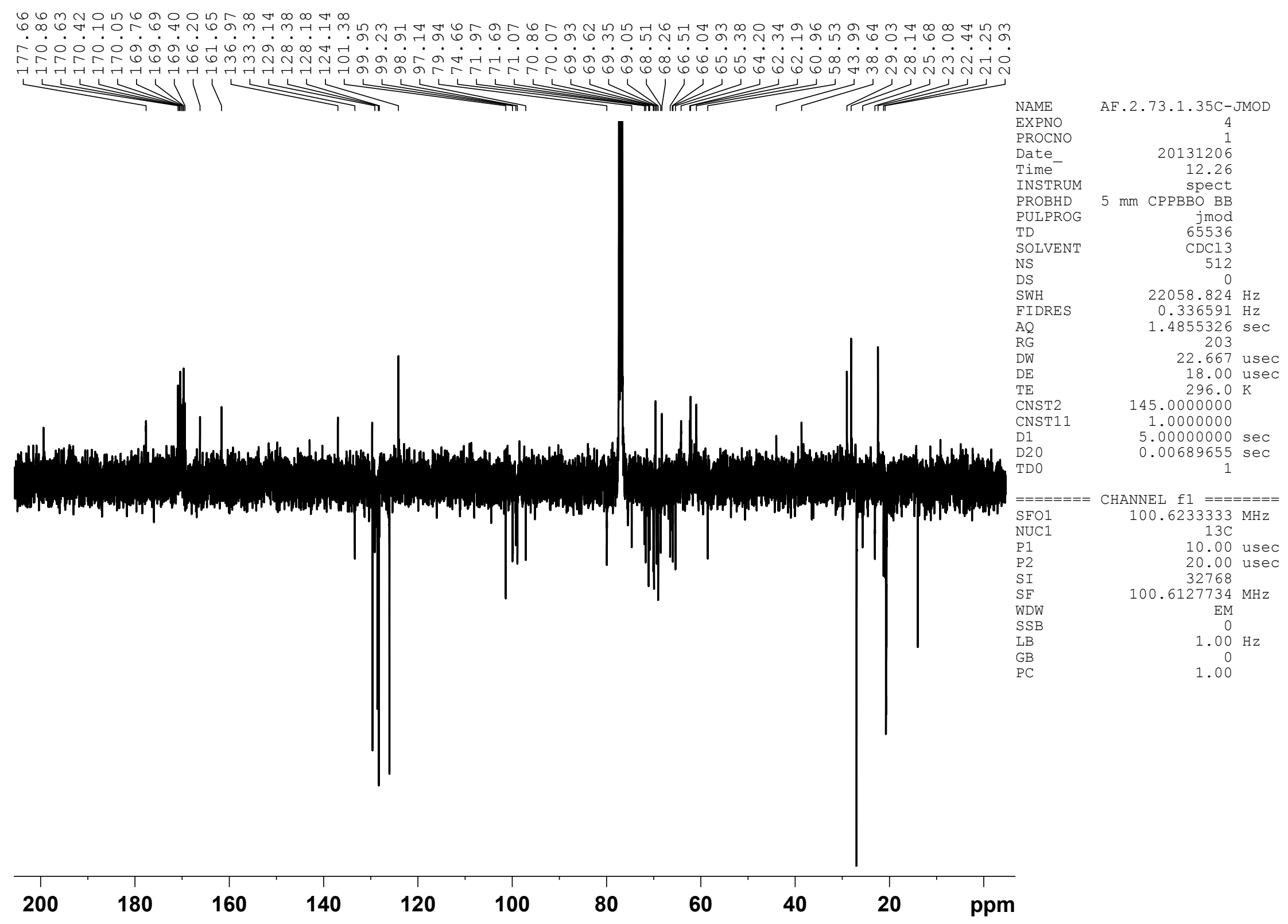


Compound 17, HSQC, $400 \mathrm{MHz}, \mathrm{CDCl}_{3}$

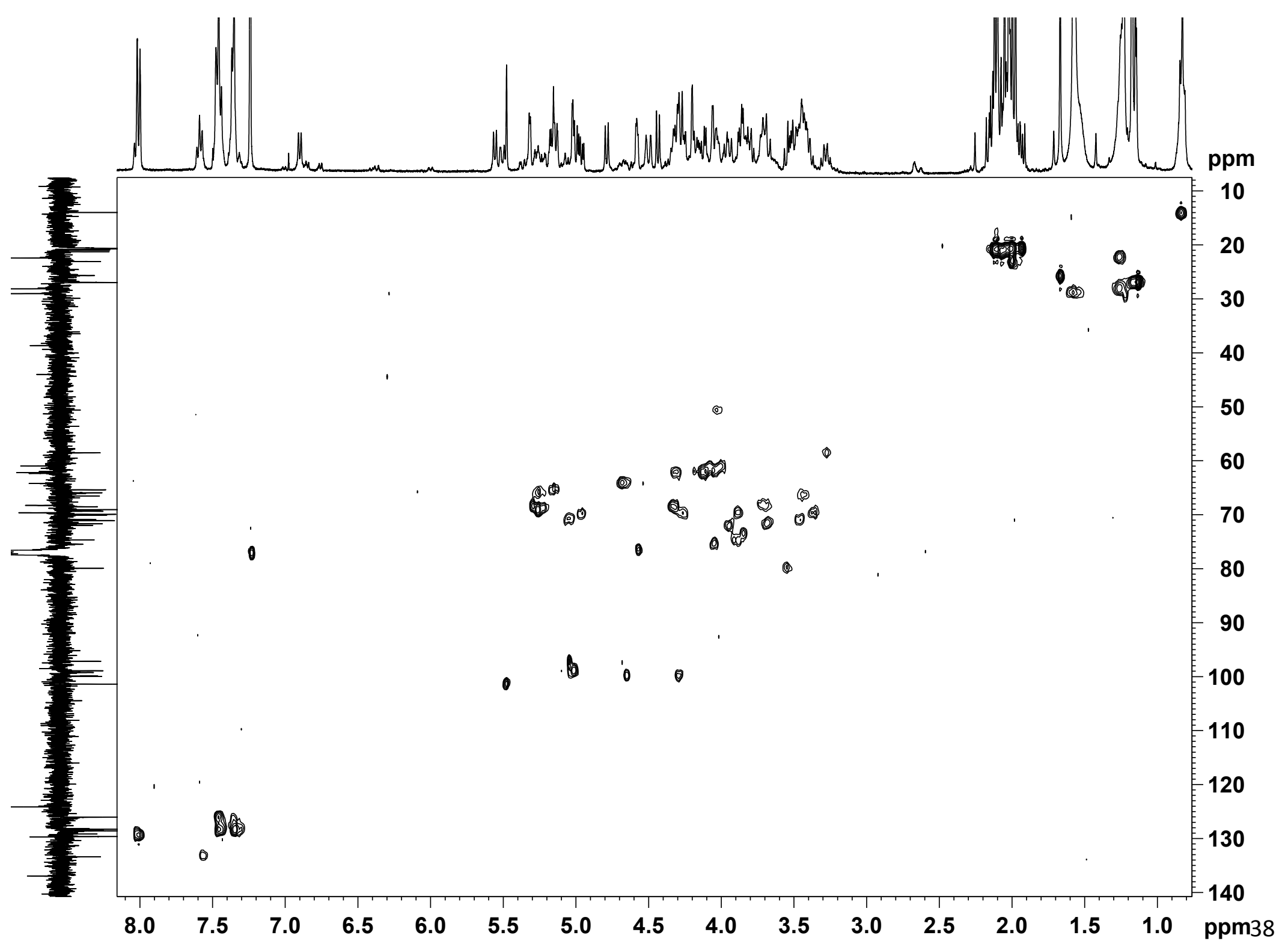


Compound 18, $600 \mathrm{MHz}, \mathrm{CDCl}_{3}$

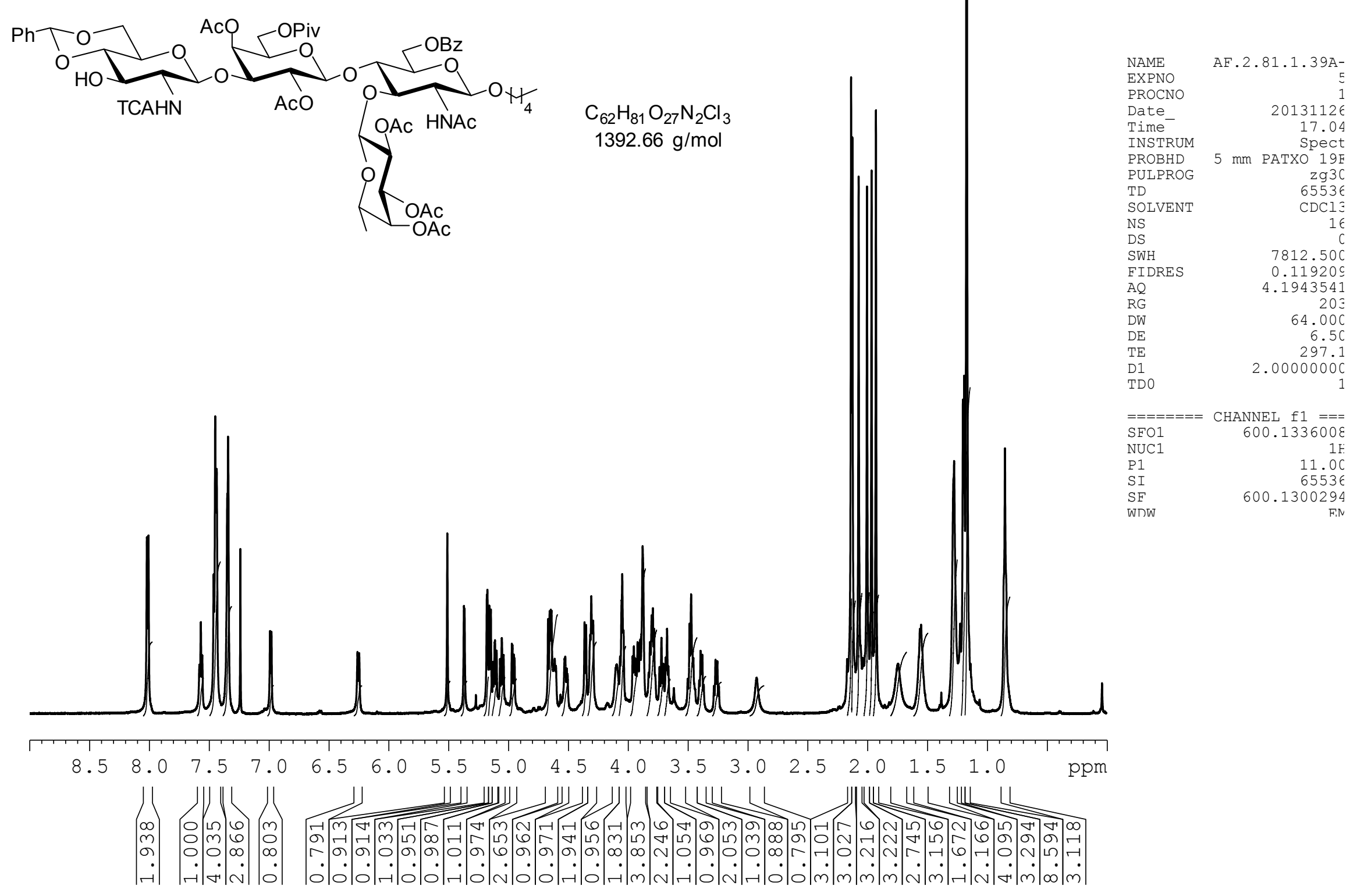


Compound 18, COSY, $400 \mathrm{MHz}, \mathrm{CDCl}_{3}$

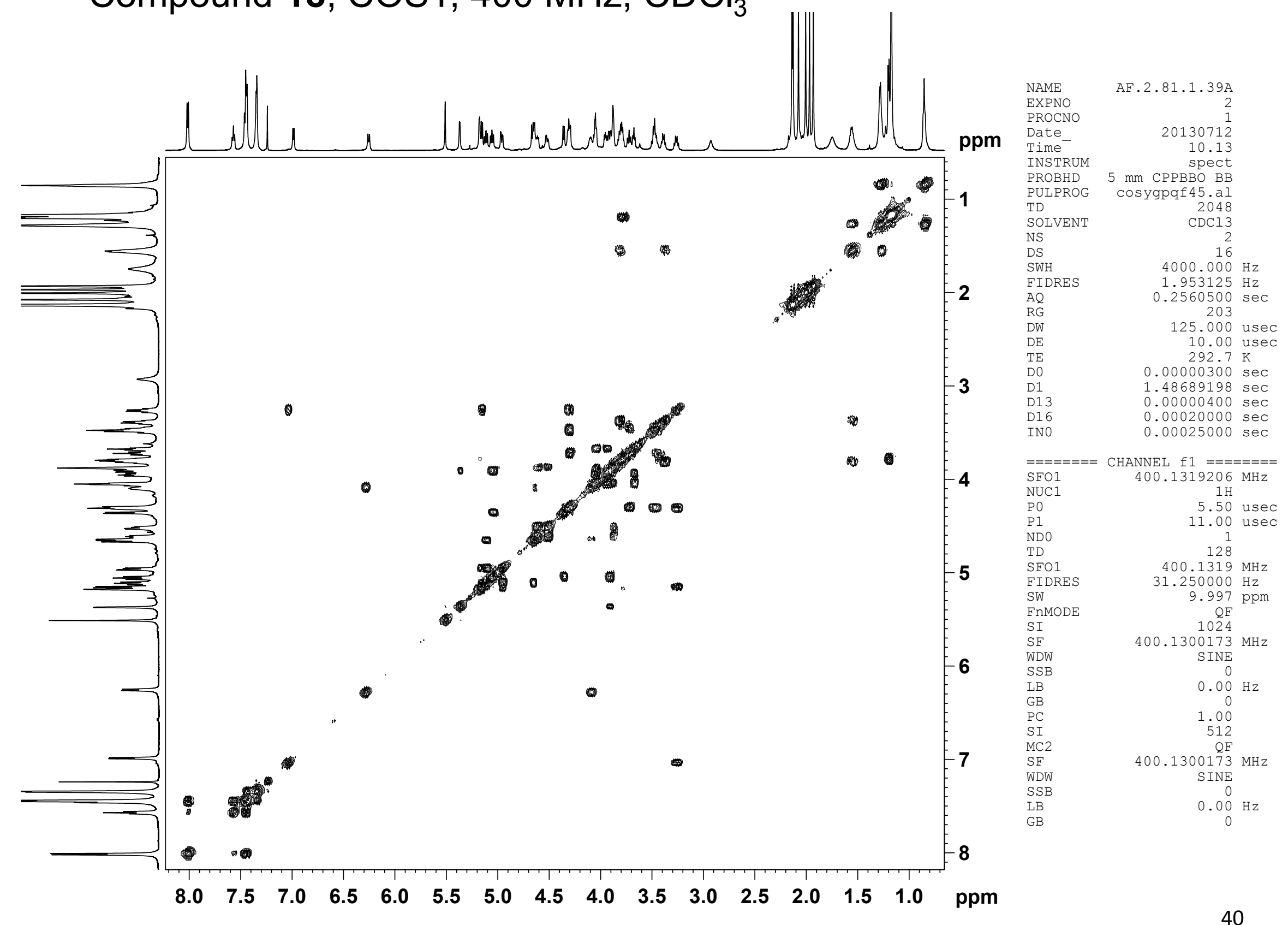


Compound 18, $101 \mathrm{MHz}, \mathrm{CDCl}_{3}$

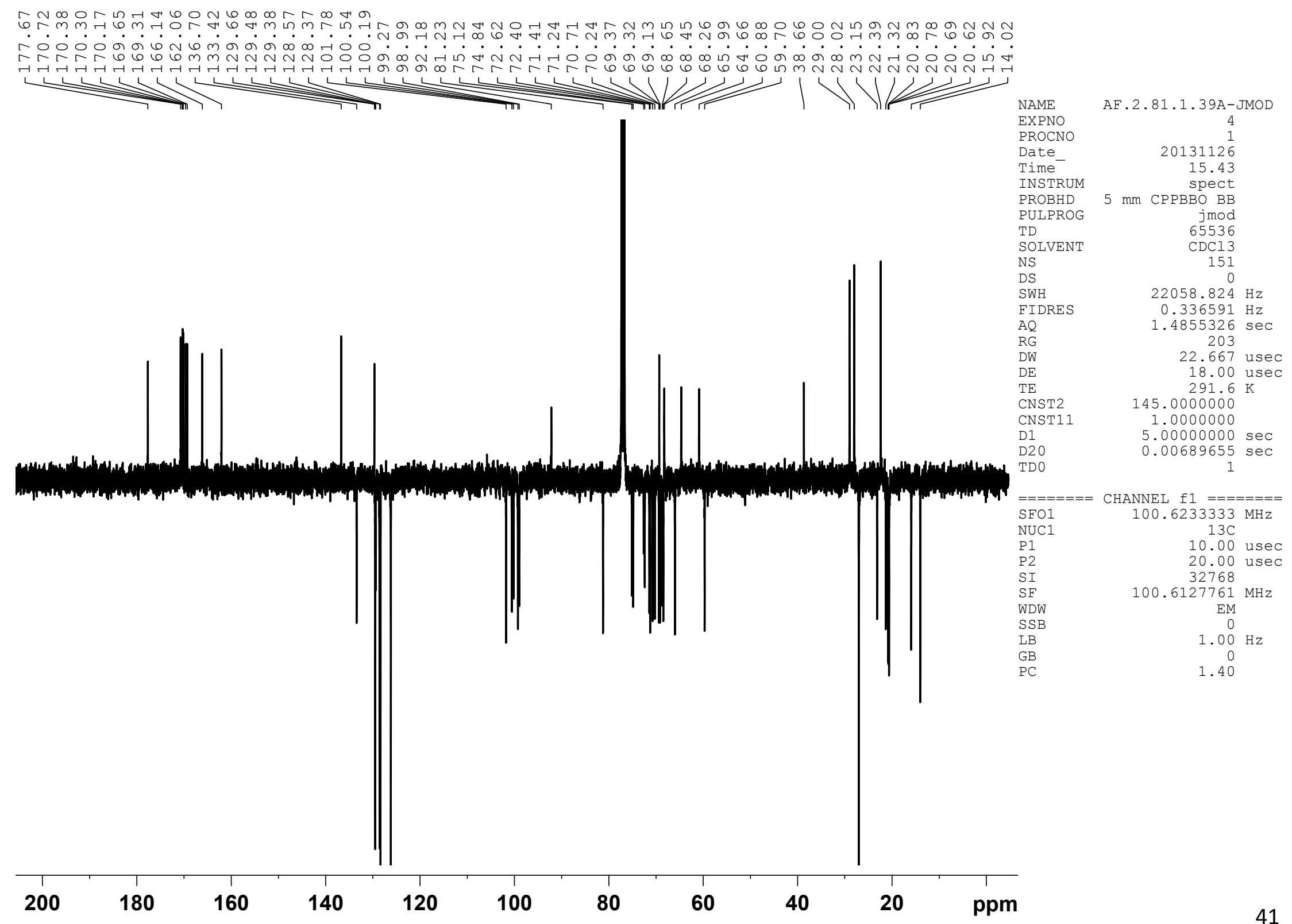


Compound 18, edited $\mathrm{HSQC}\left(\mathrm{CH} \text { and } \mathrm{CH}_{3} \text { blue, } \mathrm{CH}_{2} \text { black }\right)_{8}$ $400 \mathrm{MHz}, \mathrm{CDCl}_{3}$

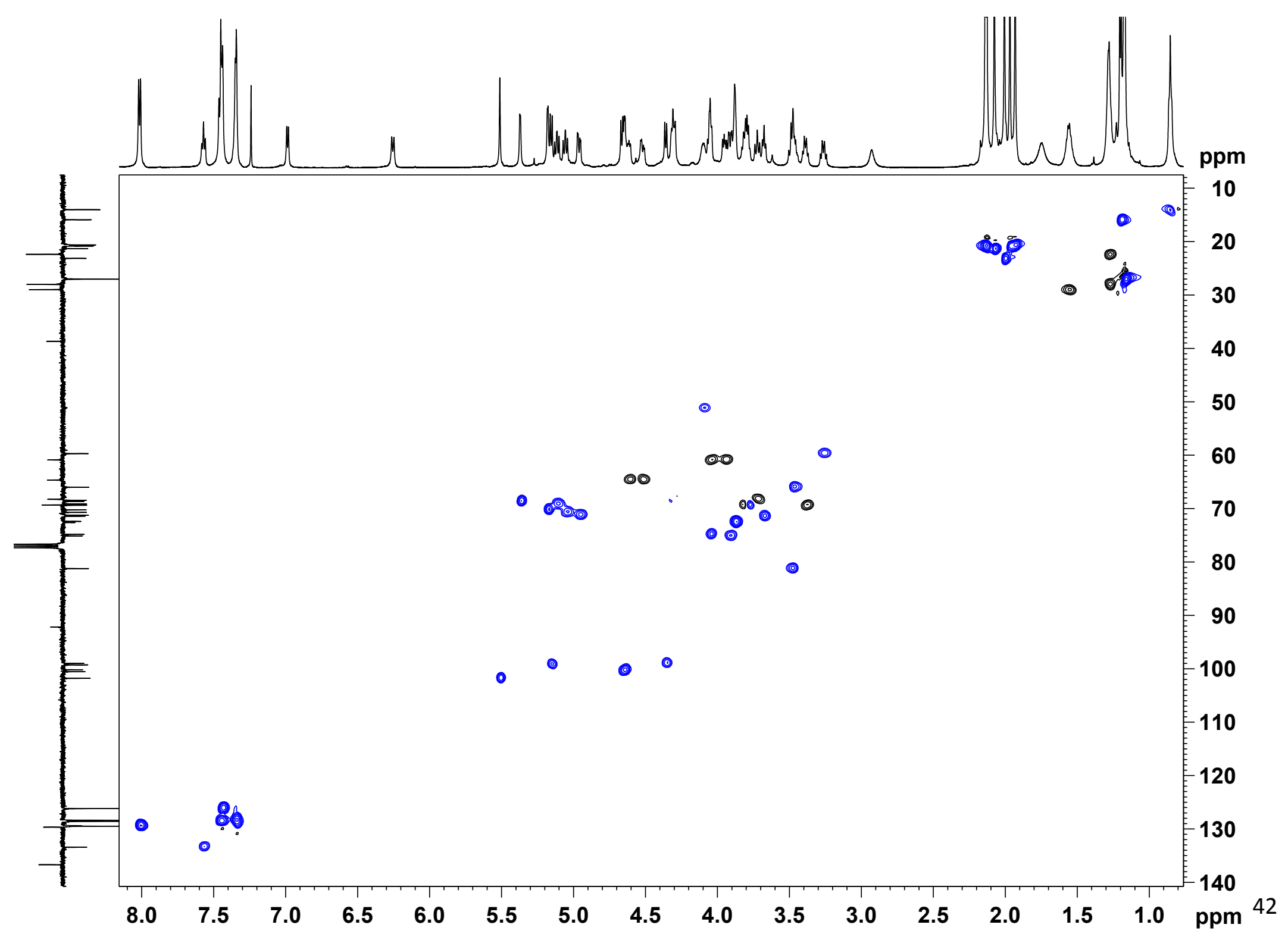




\section{Compound 19, $600 \mathrm{MHz}, \mathrm{CDCl}_{3}$}

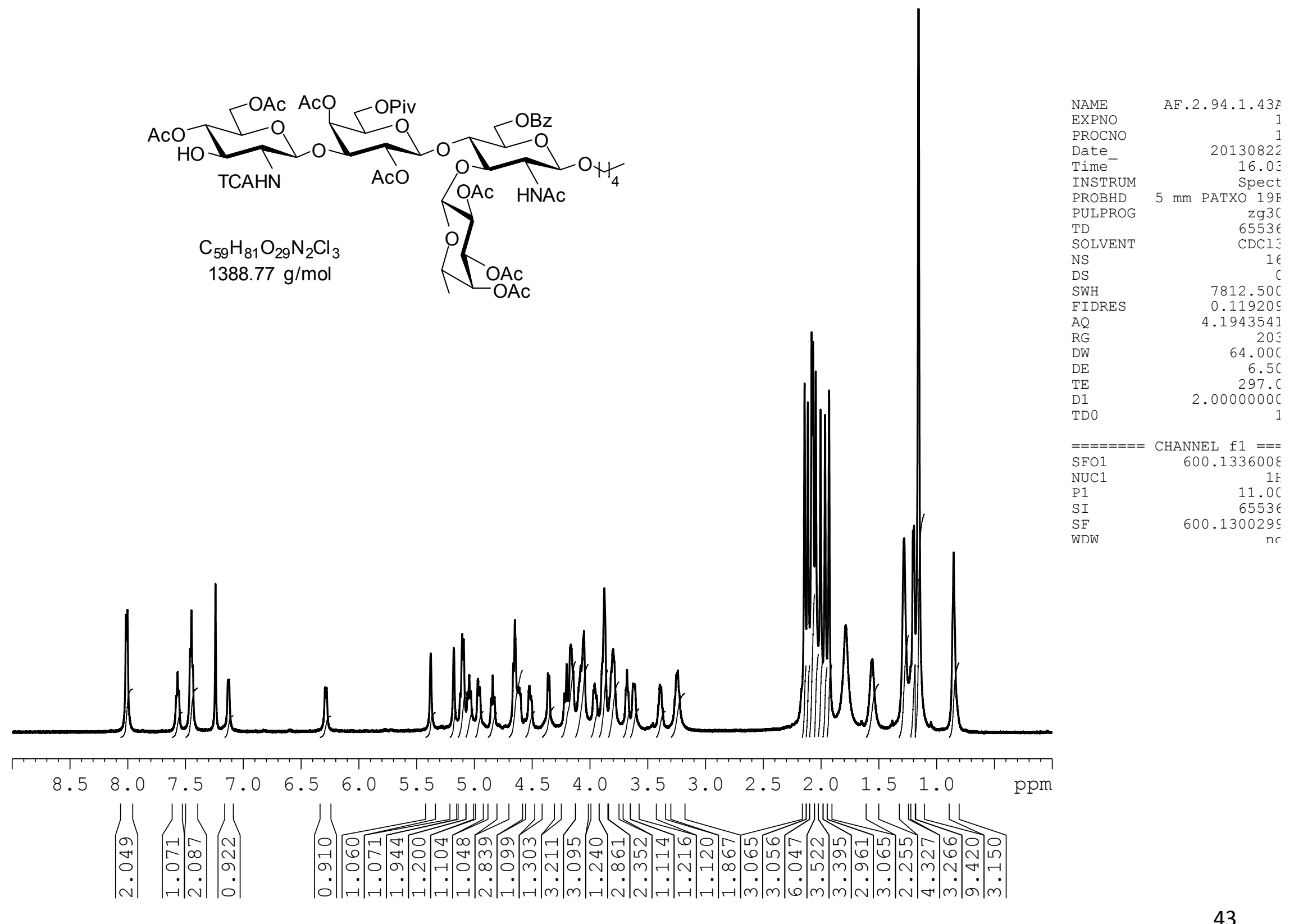


Compound 19, COSY, $600 \mathrm{MHz}, \mathrm{CDCl}_{3}$

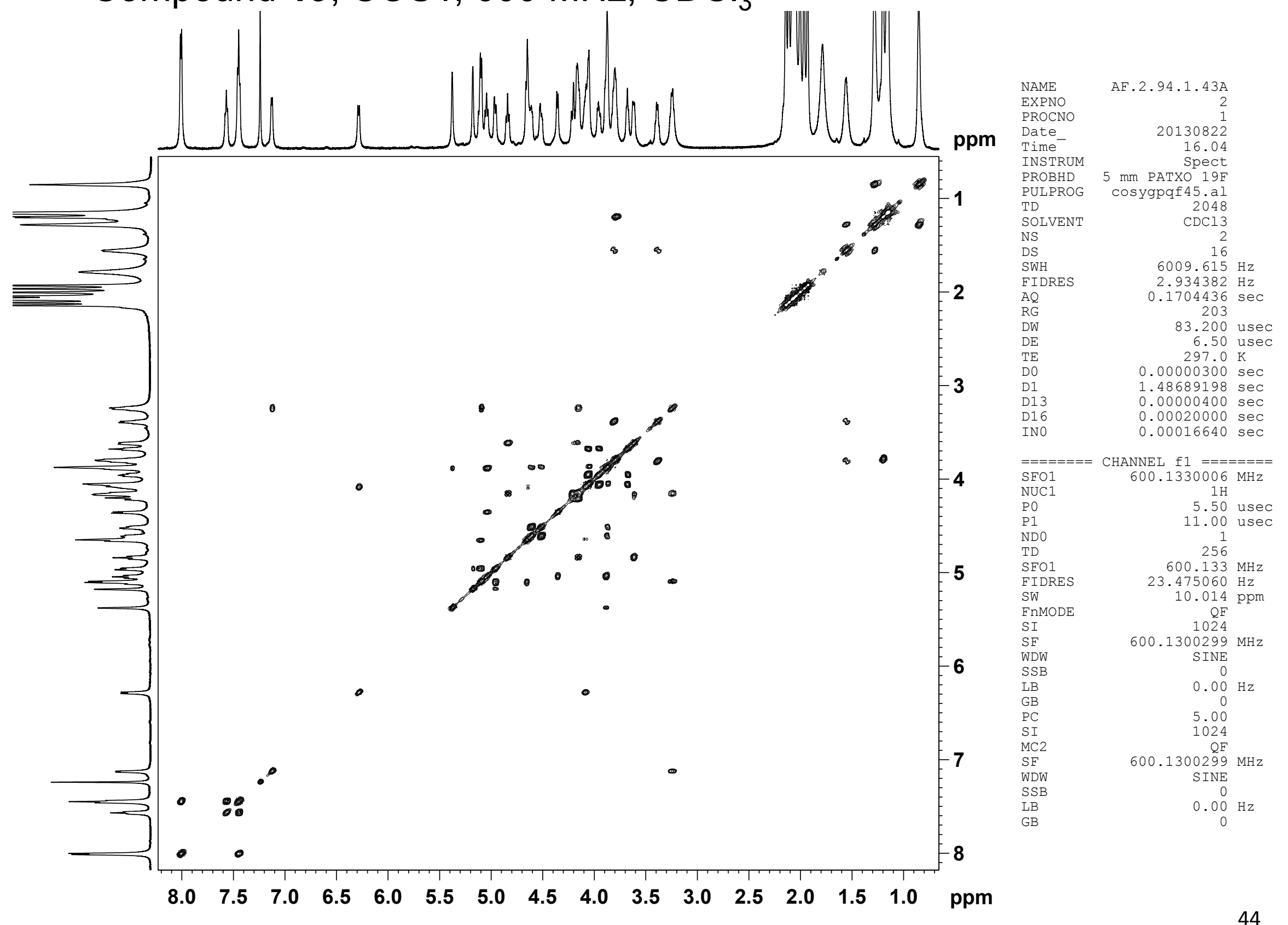




\section{Compound 19, $101 \mathrm{MHz}, \mathrm{CDCl}_{3}$}

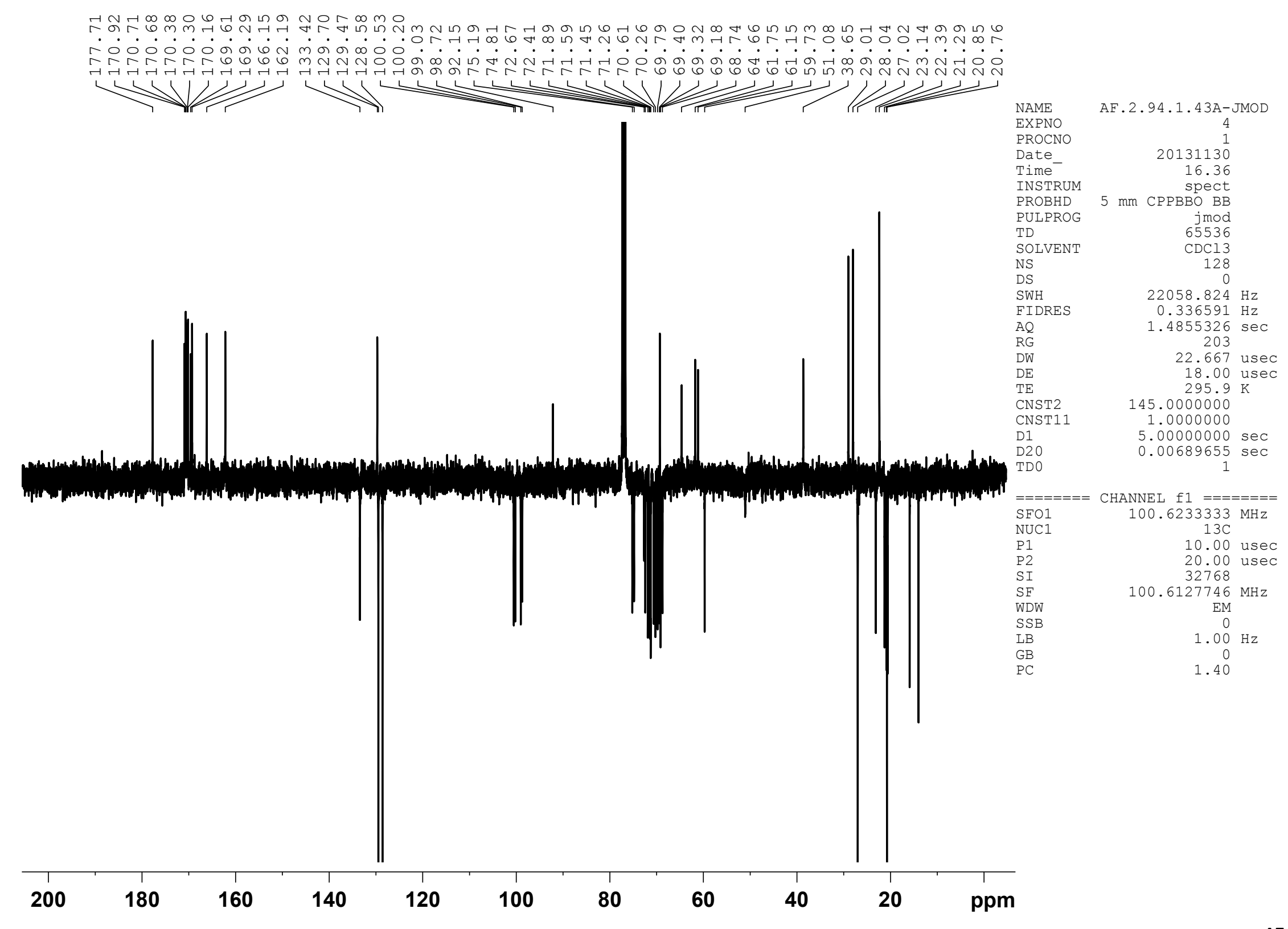


Compound 19, edited HSQC ( $\mathrm{CH}$ and $\mathrm{CH}_{3}$ blue, $\mathrm{CH}_{2}$ black), $600 \mathrm{MHz}, \mathrm{CDCl}_{3}$

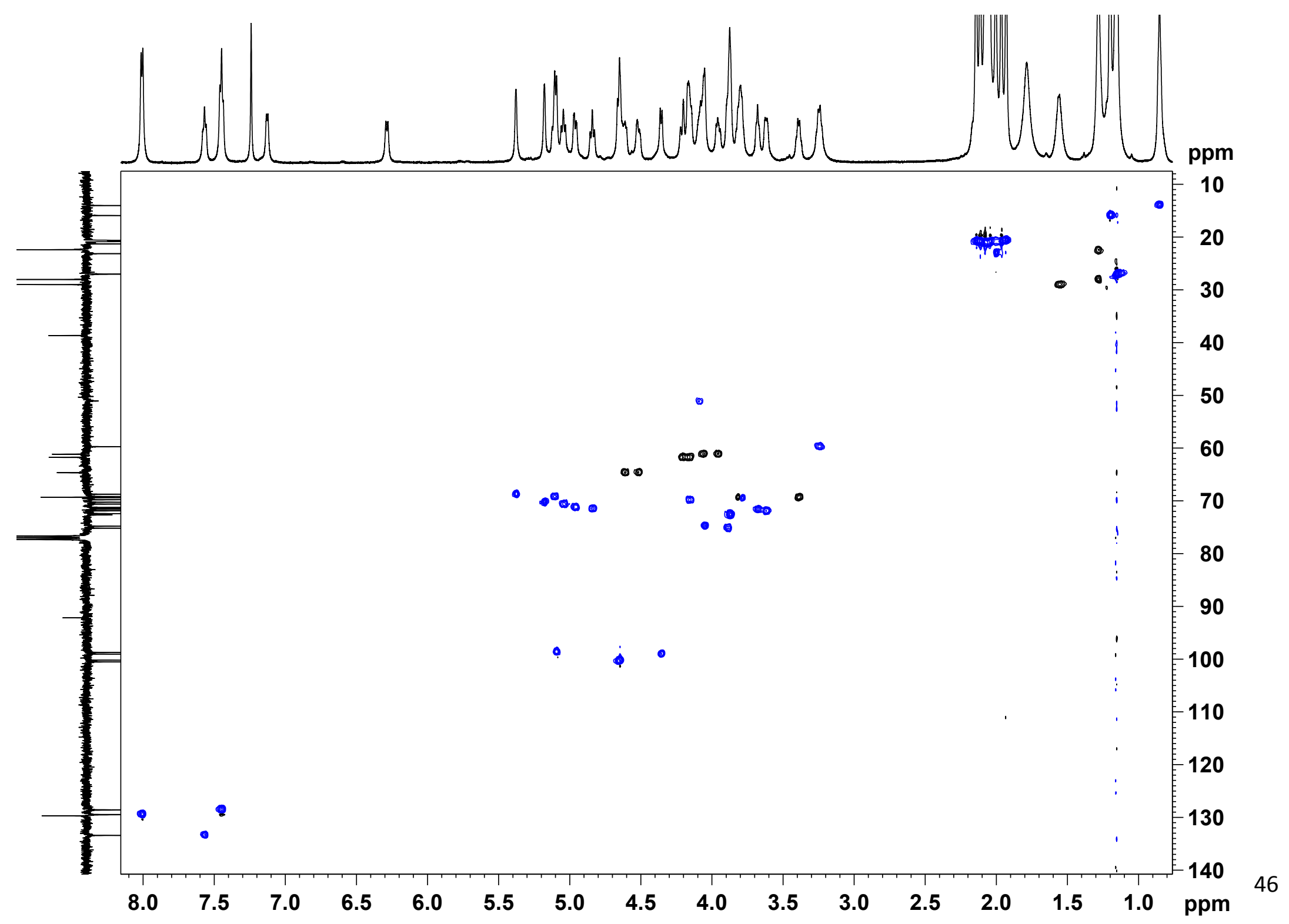


Compound 20, $600 \mathrm{MHz}, \mathrm{CDCl}_{3}$

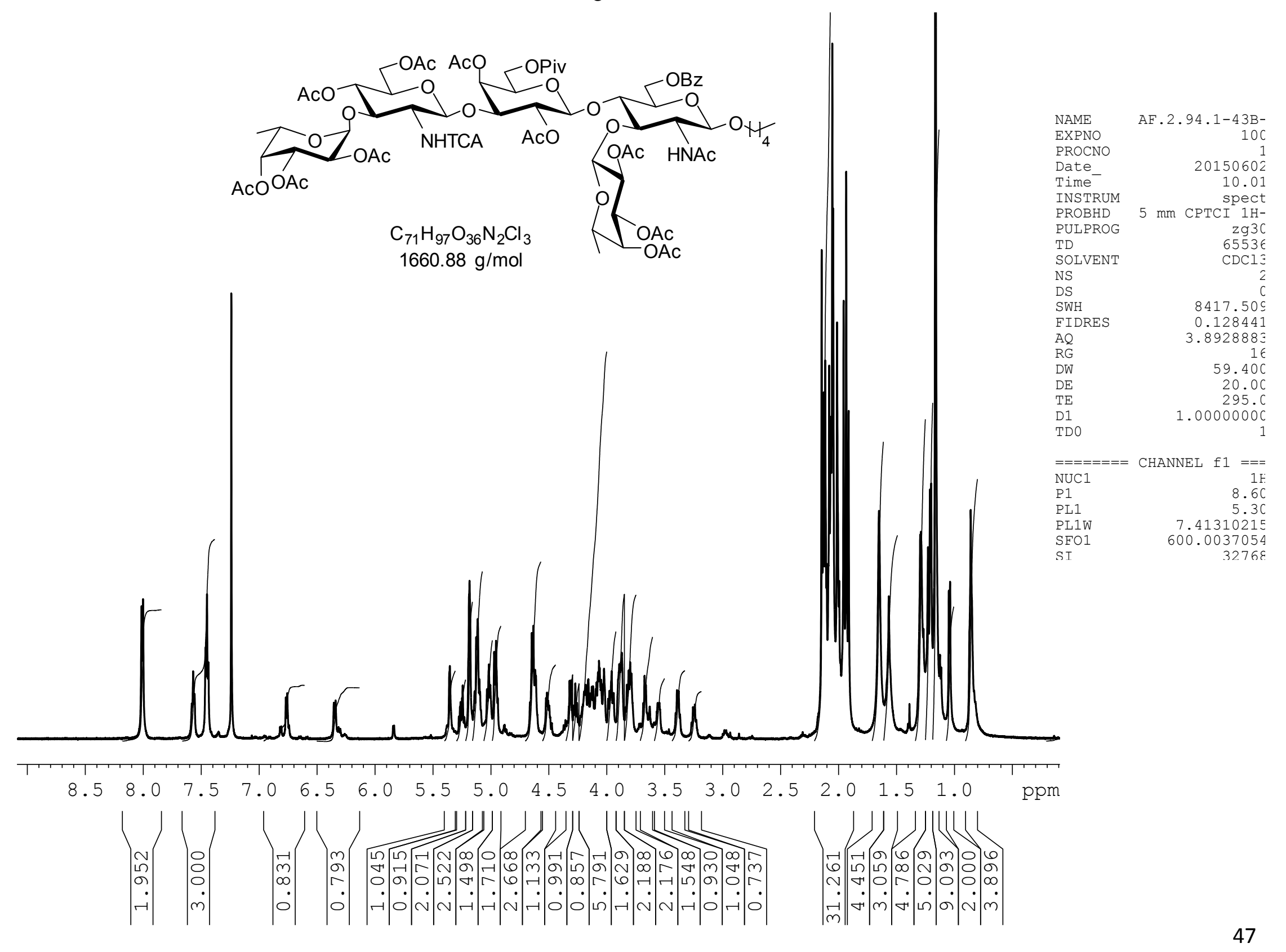


Compound 20, COSY, $600 \mathrm{MHz}, \mathrm{CDCl}_{3}$

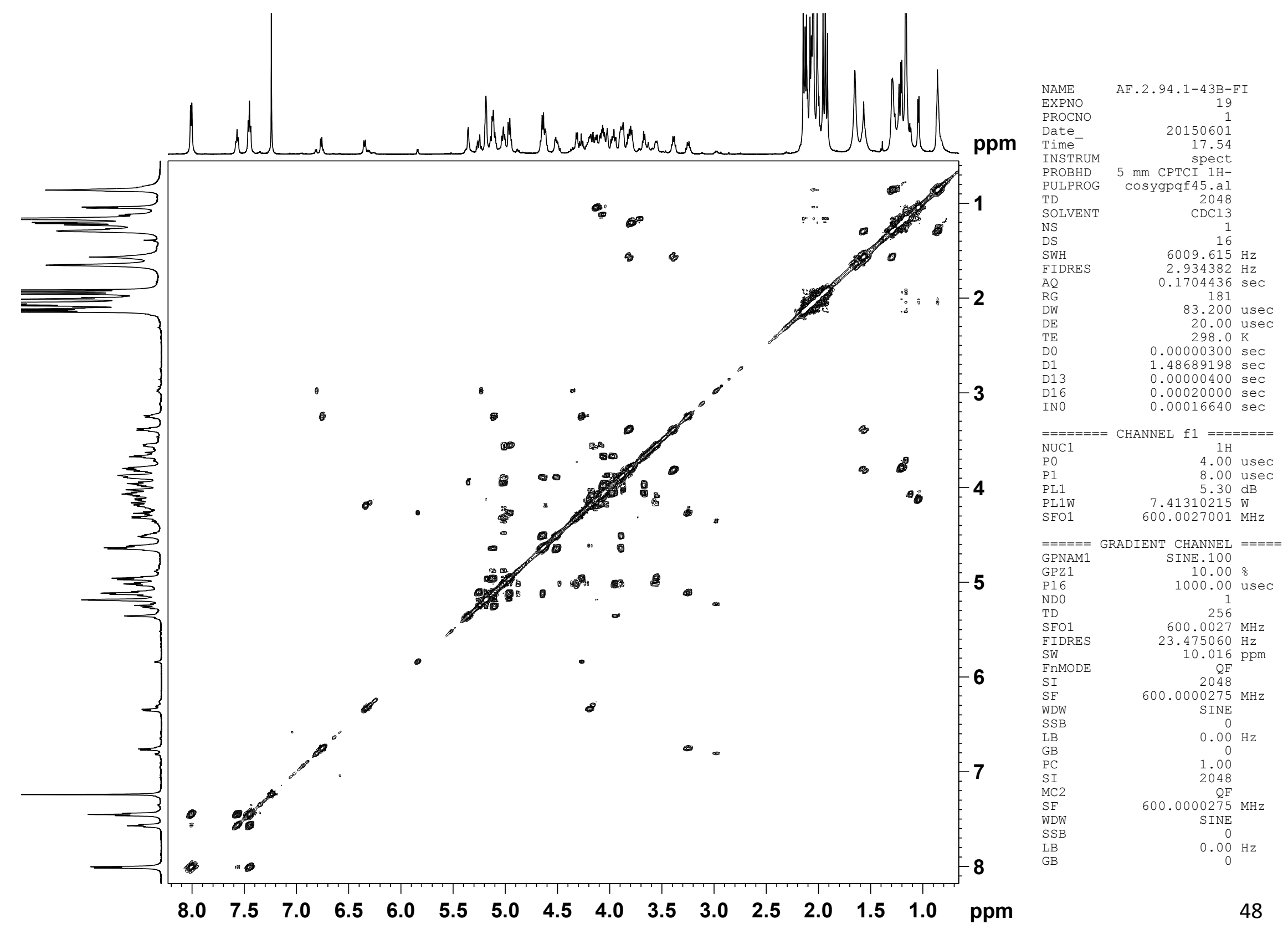




\section{Compound 20, $151 \mathrm{MHz}, \mathrm{CDCl}_{3}$}

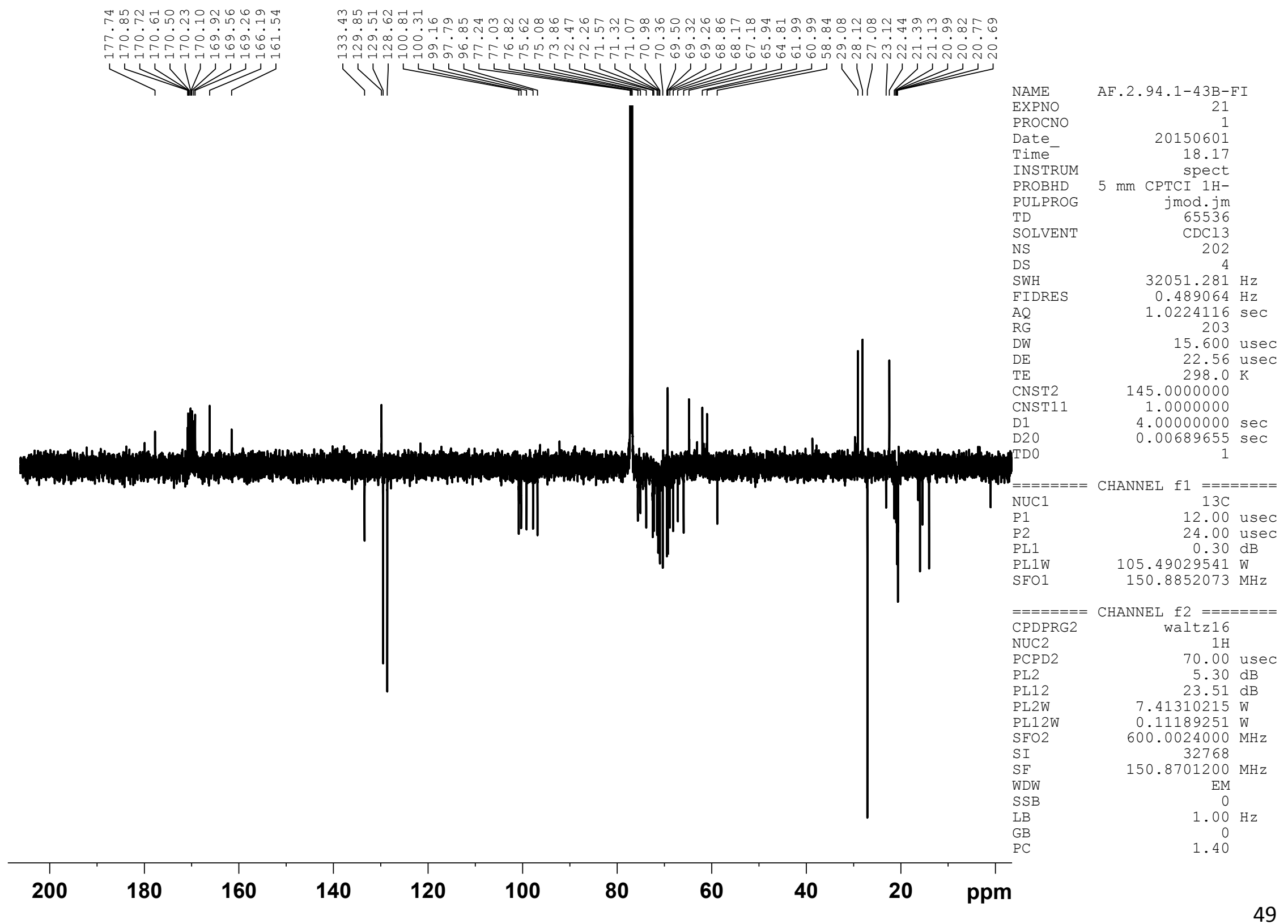




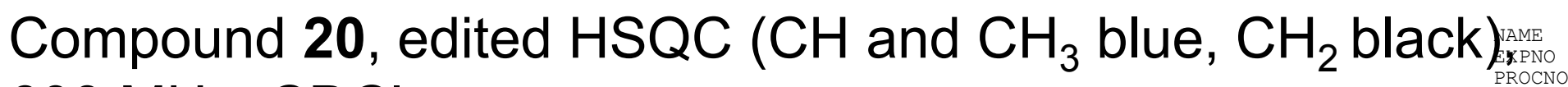
$600 \mathrm{MHz}, \mathrm{CDCl}_{3}$

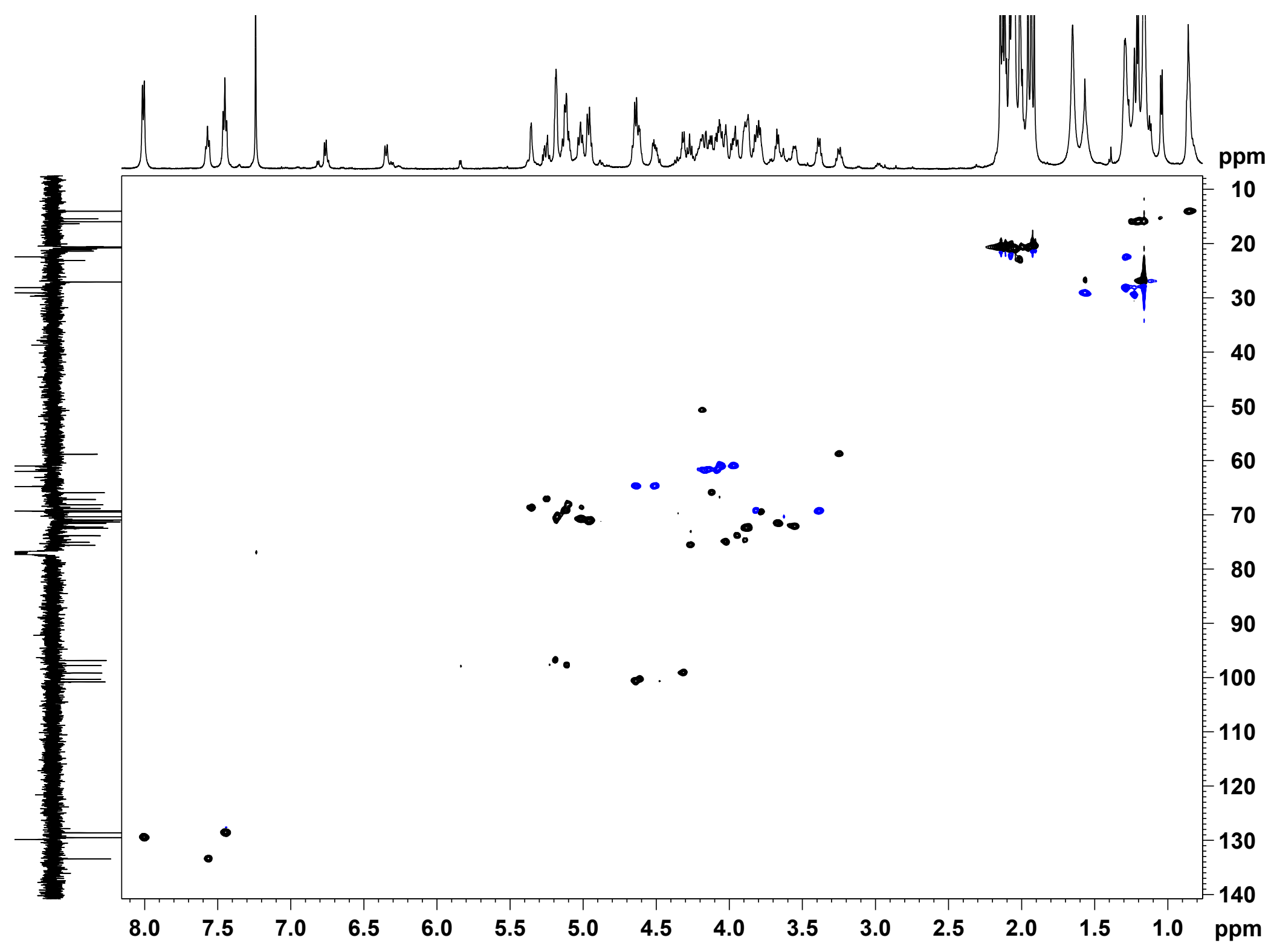


Compound 21, $400 \mathrm{MHz}, \mathrm{CDCl}_{3}$

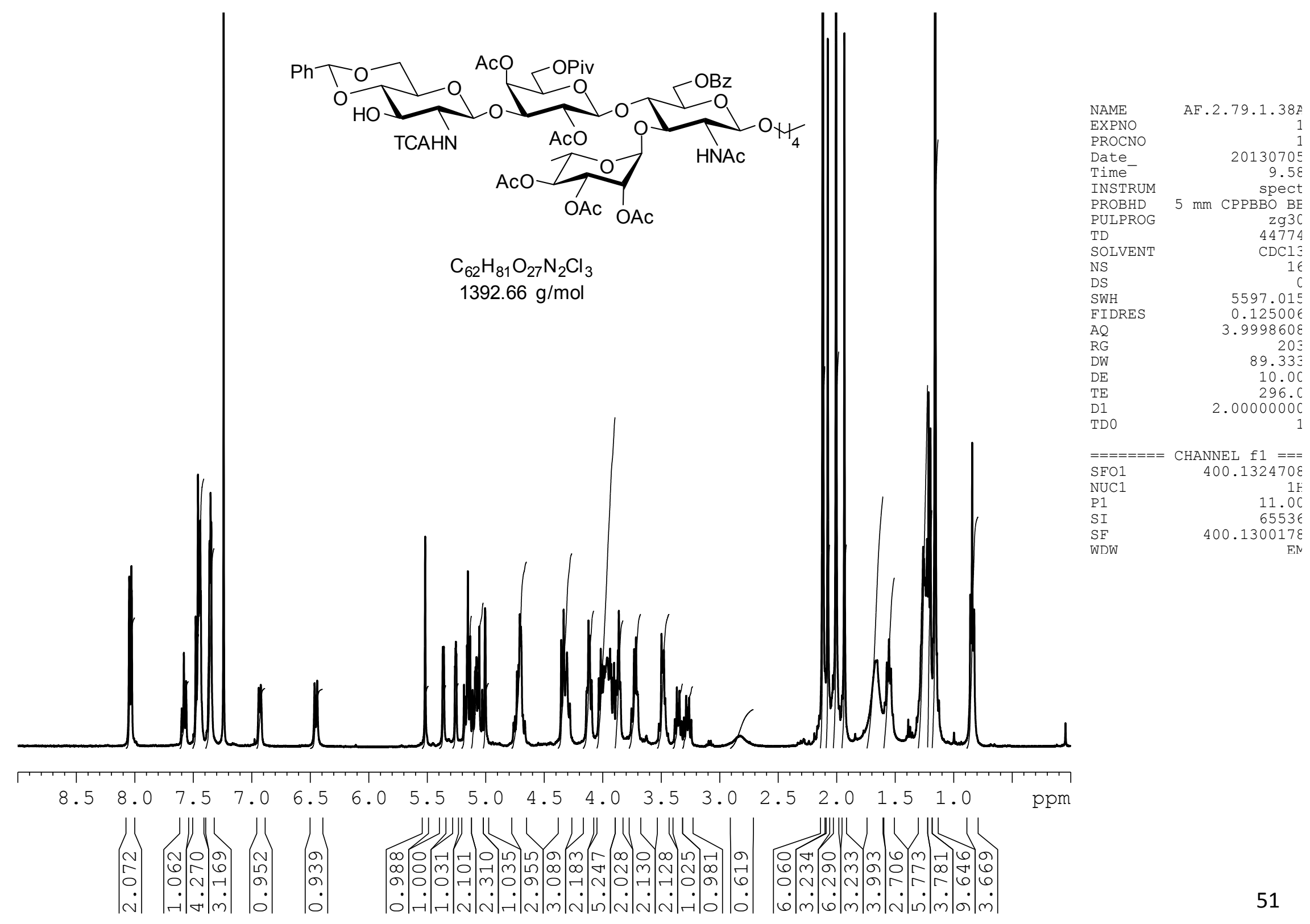


Compound 21, COSY, $400 \mathrm{MHz}, \mathrm{CDCl}_{3}$

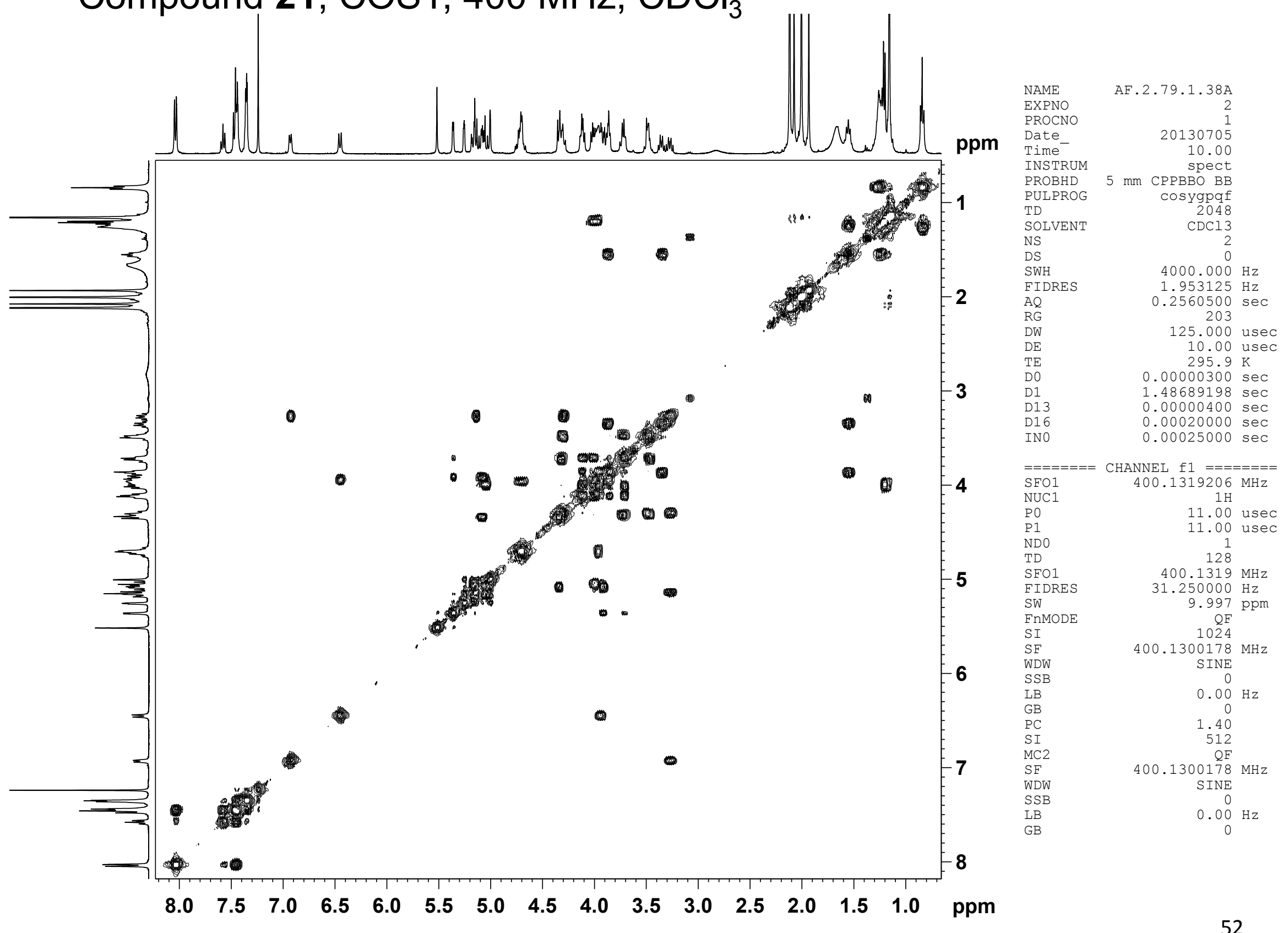


Compound 21, $101 \mathrm{MHz}, \mathrm{CDCl}_{3}$

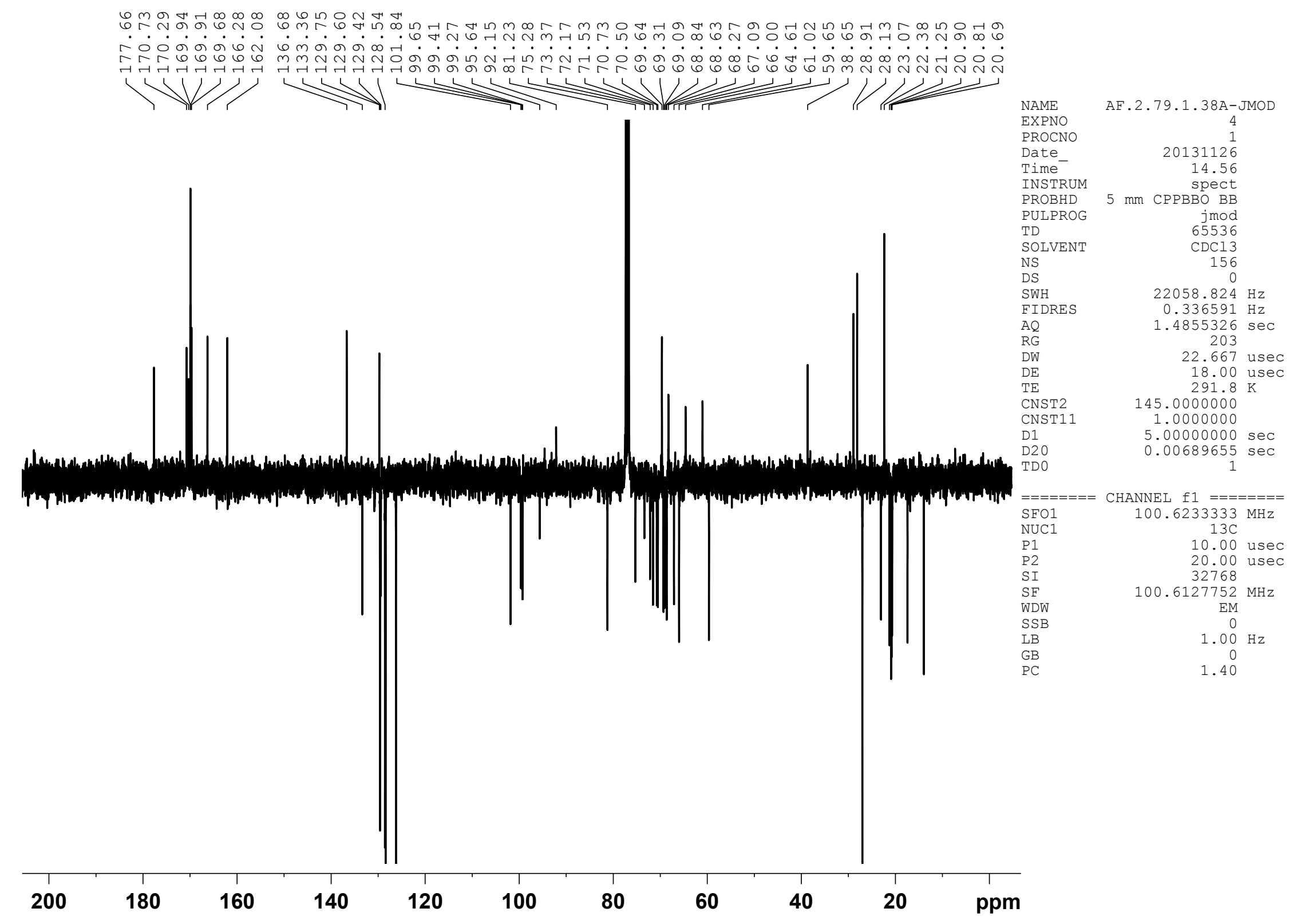


Compound 21, edited HSQC ( $\mathrm{CH}$ and $\mathrm{CH}_{3}$ blue, $\mathrm{CH}_{2}$ black) $400 \mathrm{MHz}, \mathrm{CDCl}_{3}$

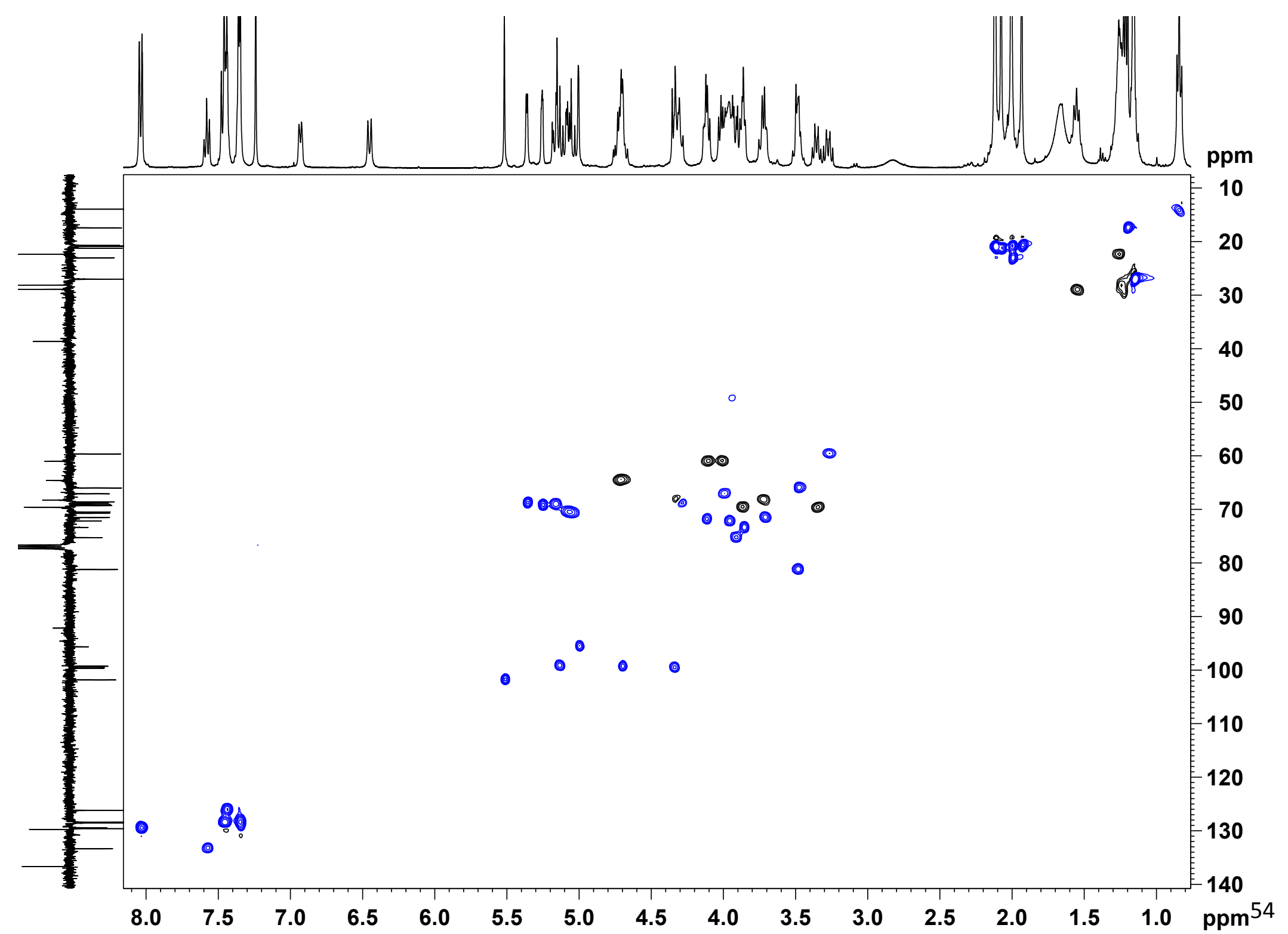




\section{Compound 22, $600 \mathrm{MHz}, \mathrm{CDCl}_{3}$}
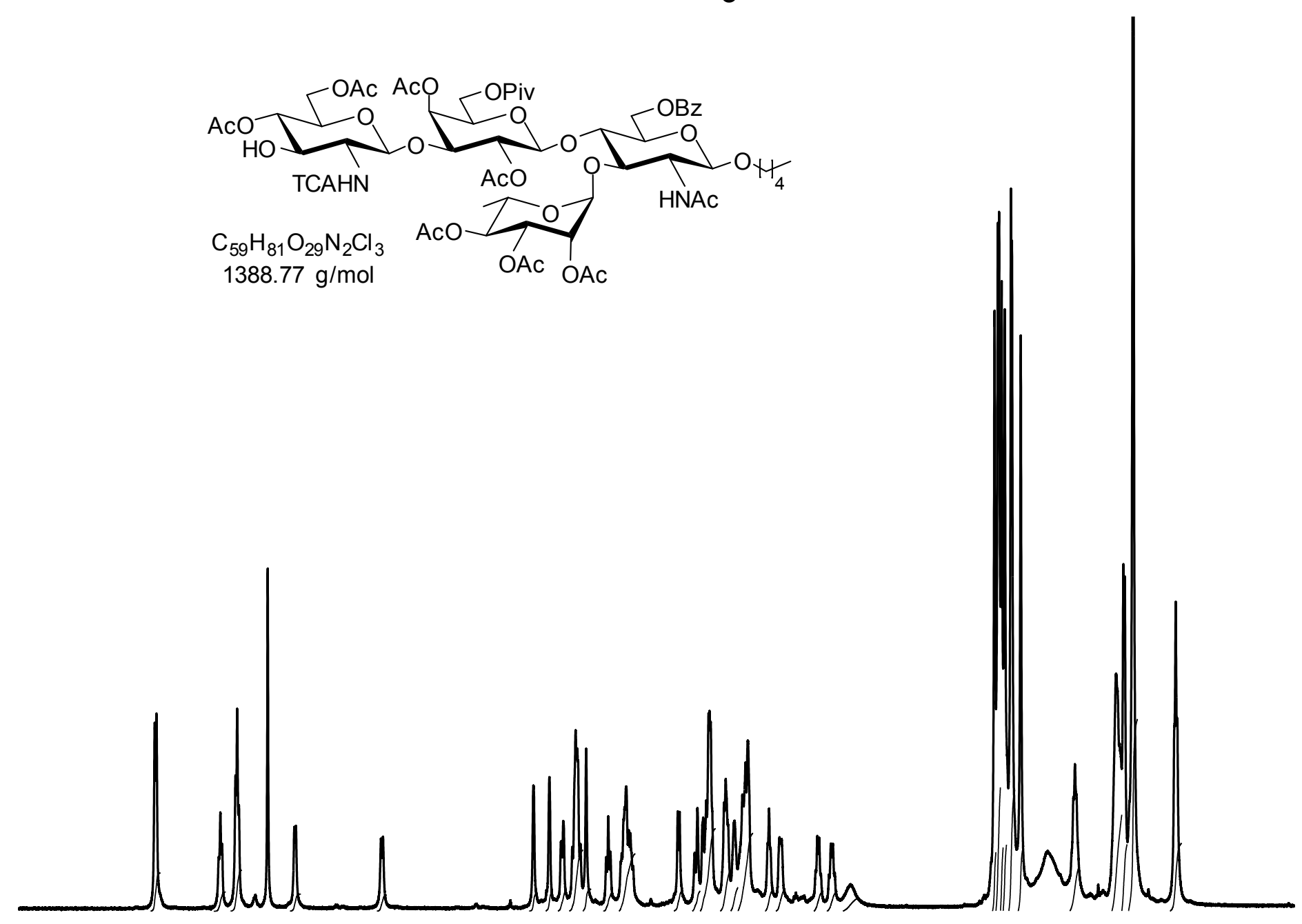

Current Data Parameters NAME EXNO PROCNO

F2 - Acquisition Parame Date_ 20130822 INSTRUM S Spect PROBHD $5 \mathrm{~mm}$ PATXO $19 \mathrm{~F}$ 
Compound 22, COSY, $600 \mathrm{MHz}, \mathrm{CDCl}_{3}$

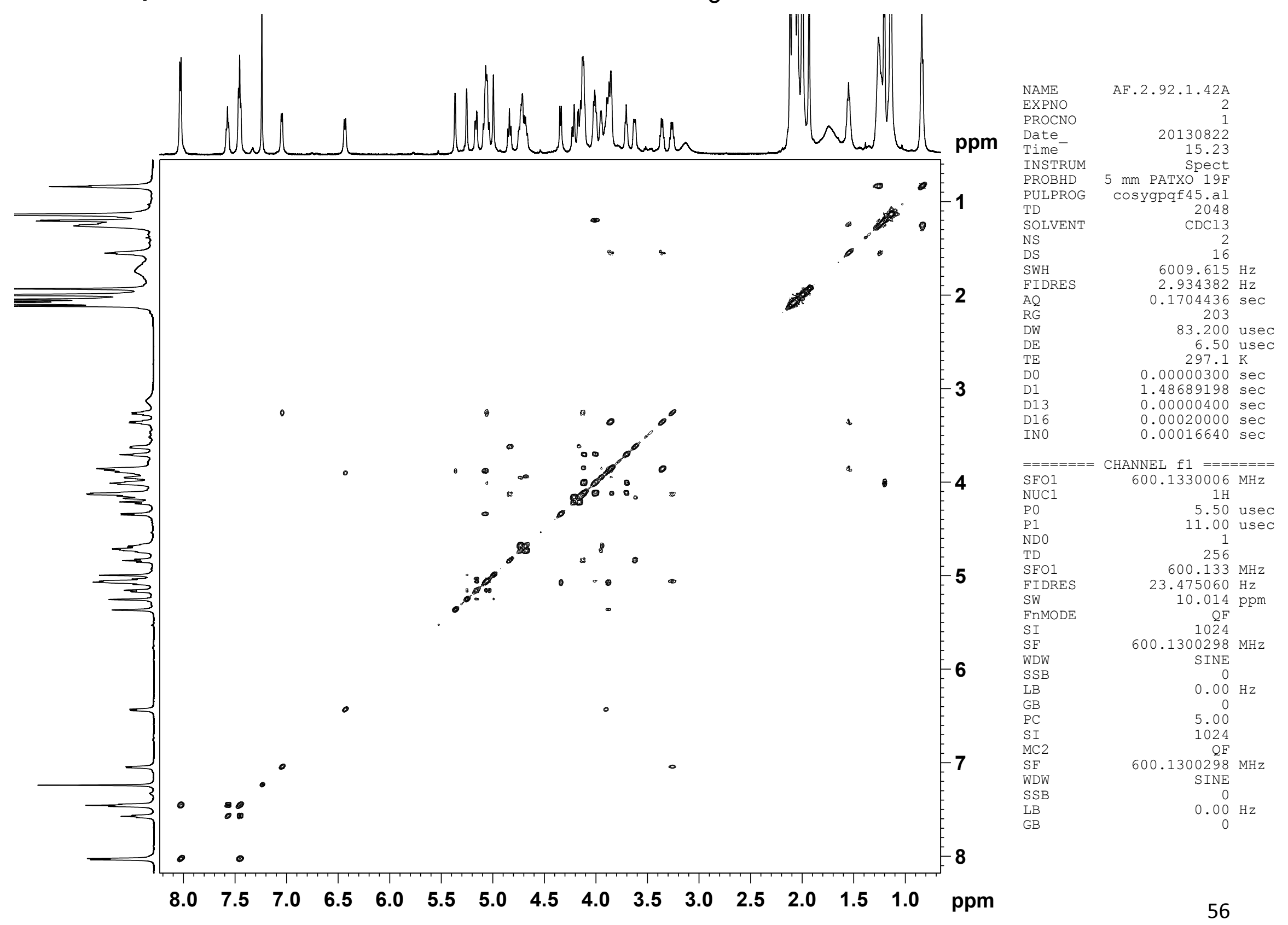




\section{Compound 22, $101 \mathrm{MHz}, \mathrm{CDCl}_{3}$}

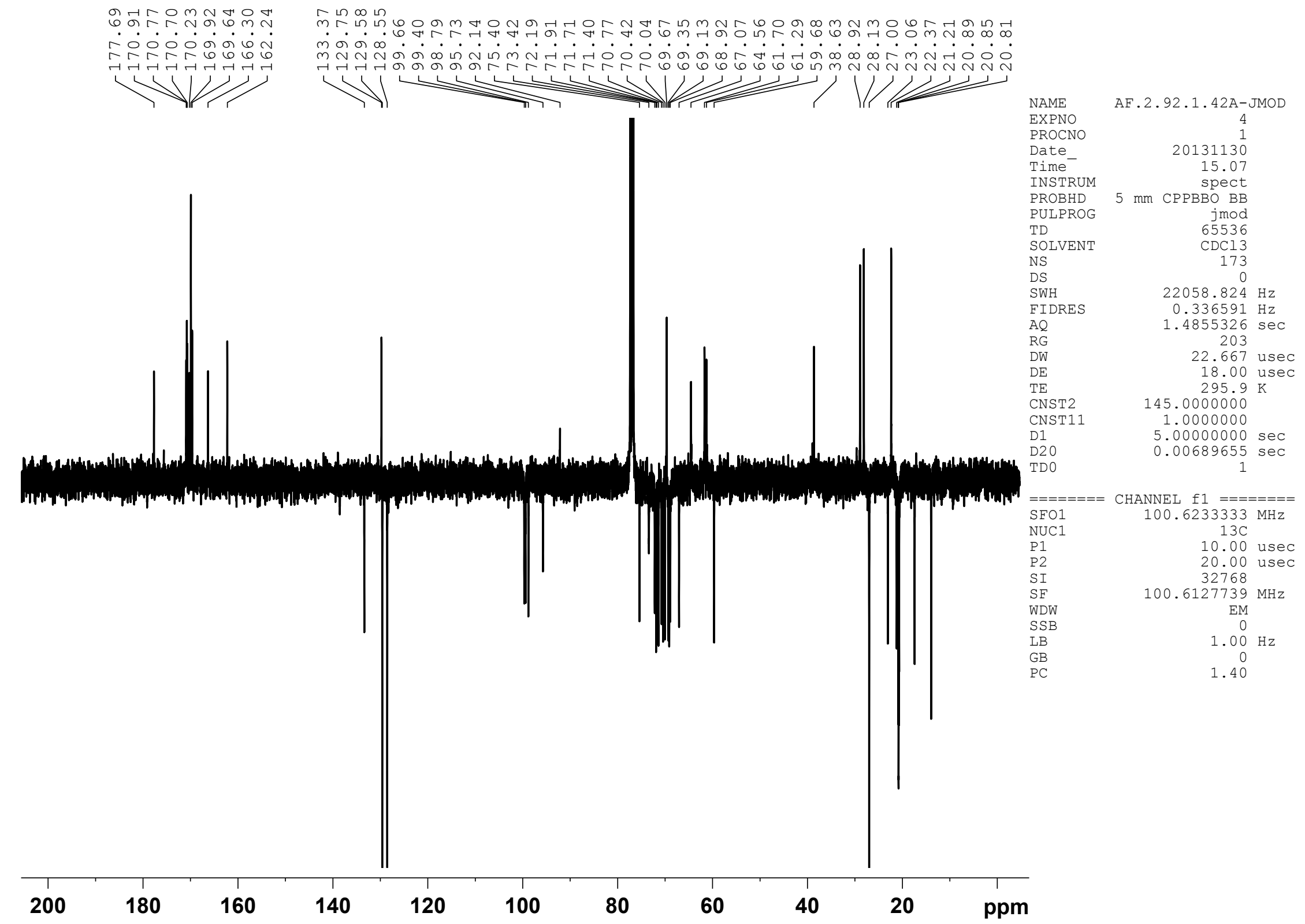


Compound 22, edited HSQC $\left(\mathrm{CH}\right.$ and $\mathrm{CH}_{3}$ blue, $\mathrm{CH}_{2}$ black), $600 \mathrm{MHz}, \mathrm{CDCl}_{3}$

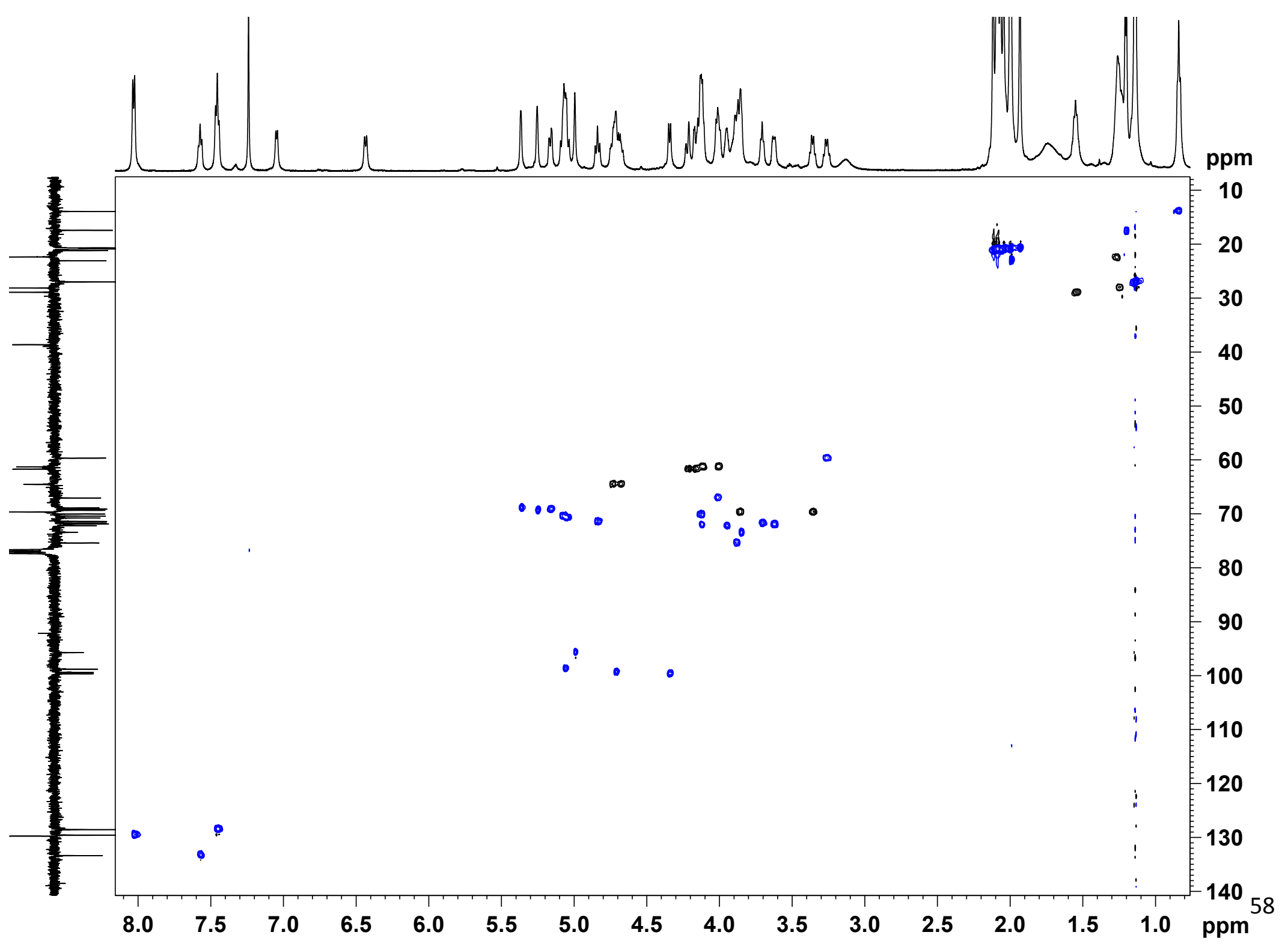


Compound 23, $400 \mathrm{MHz}, \mathrm{CDCl}_{3}, \sim 80 \%$ pure (see text, $20 \%$ suspected orthoester)

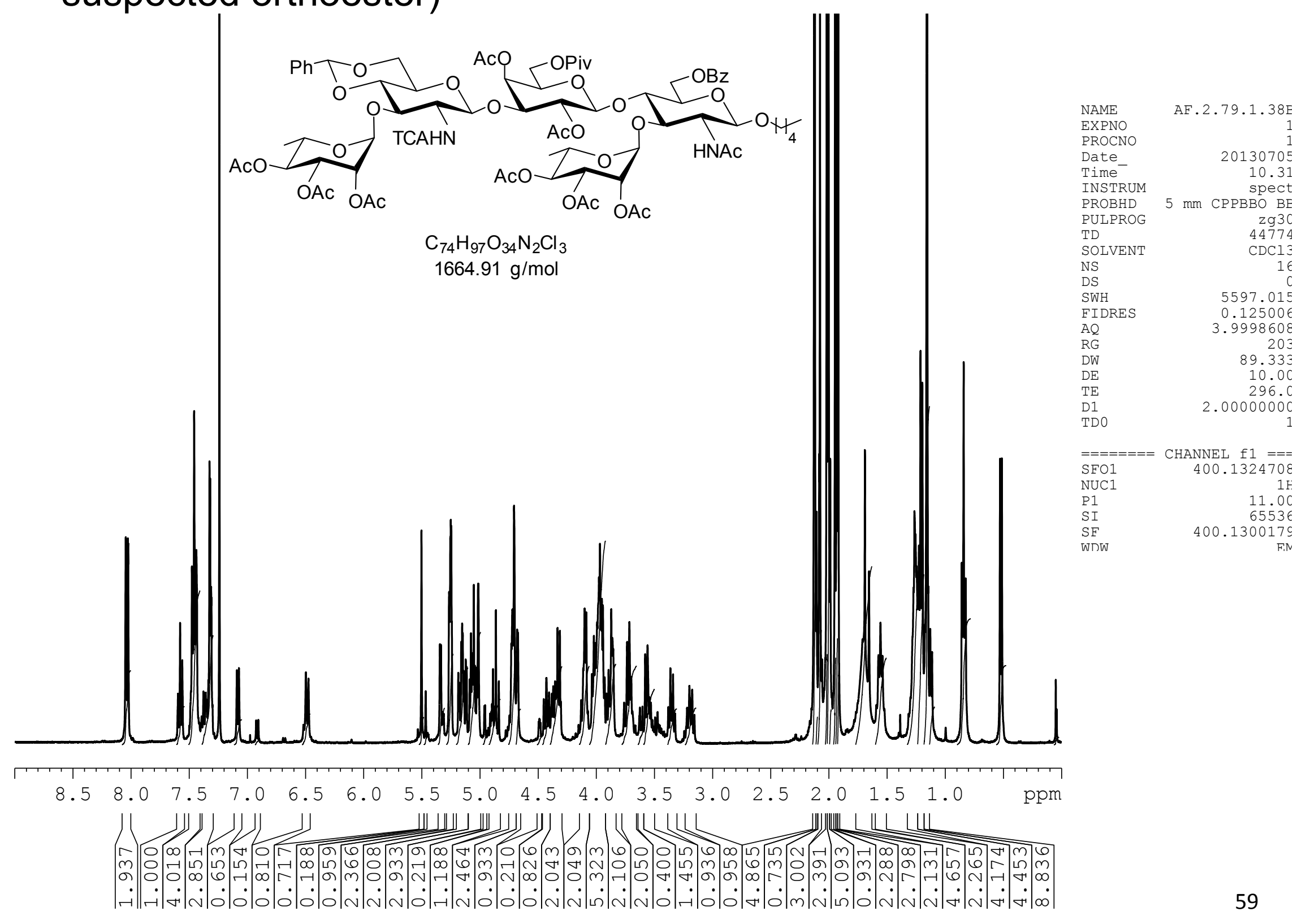


Compound 23, COSY, $400 \mathrm{MHz}, \mathrm{CDCl}_{3}, \sim 80 \%$ pure (see text, $20 \%$ suspected orthoester)

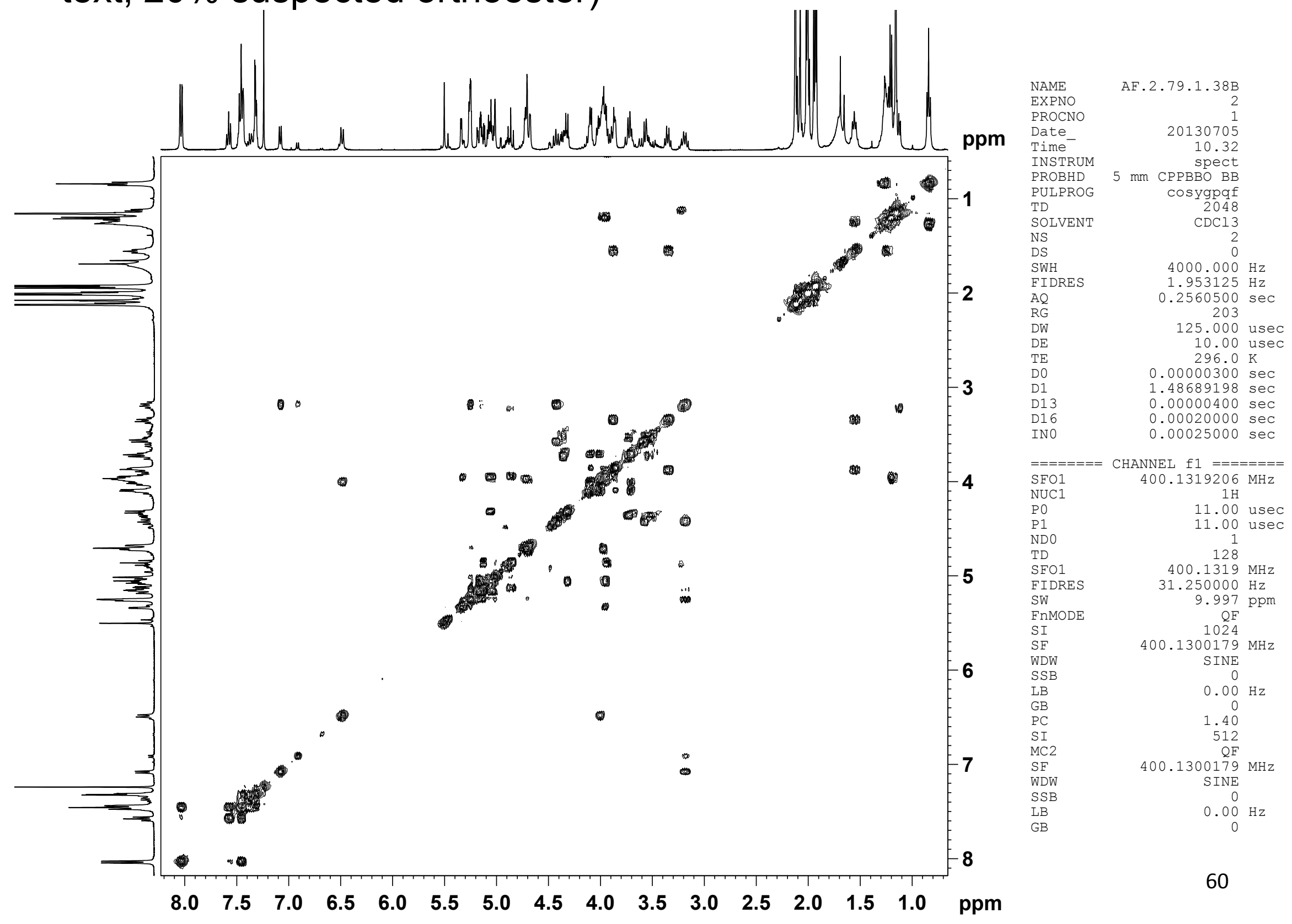


Compound 23, $101 \mathrm{MHz}, \mathrm{CDCl}_{3}, \sim 80 \%$ pure (see text, $20 \%$ suspected orthoester, quat $123.57 \mathrm{ppm}$ )

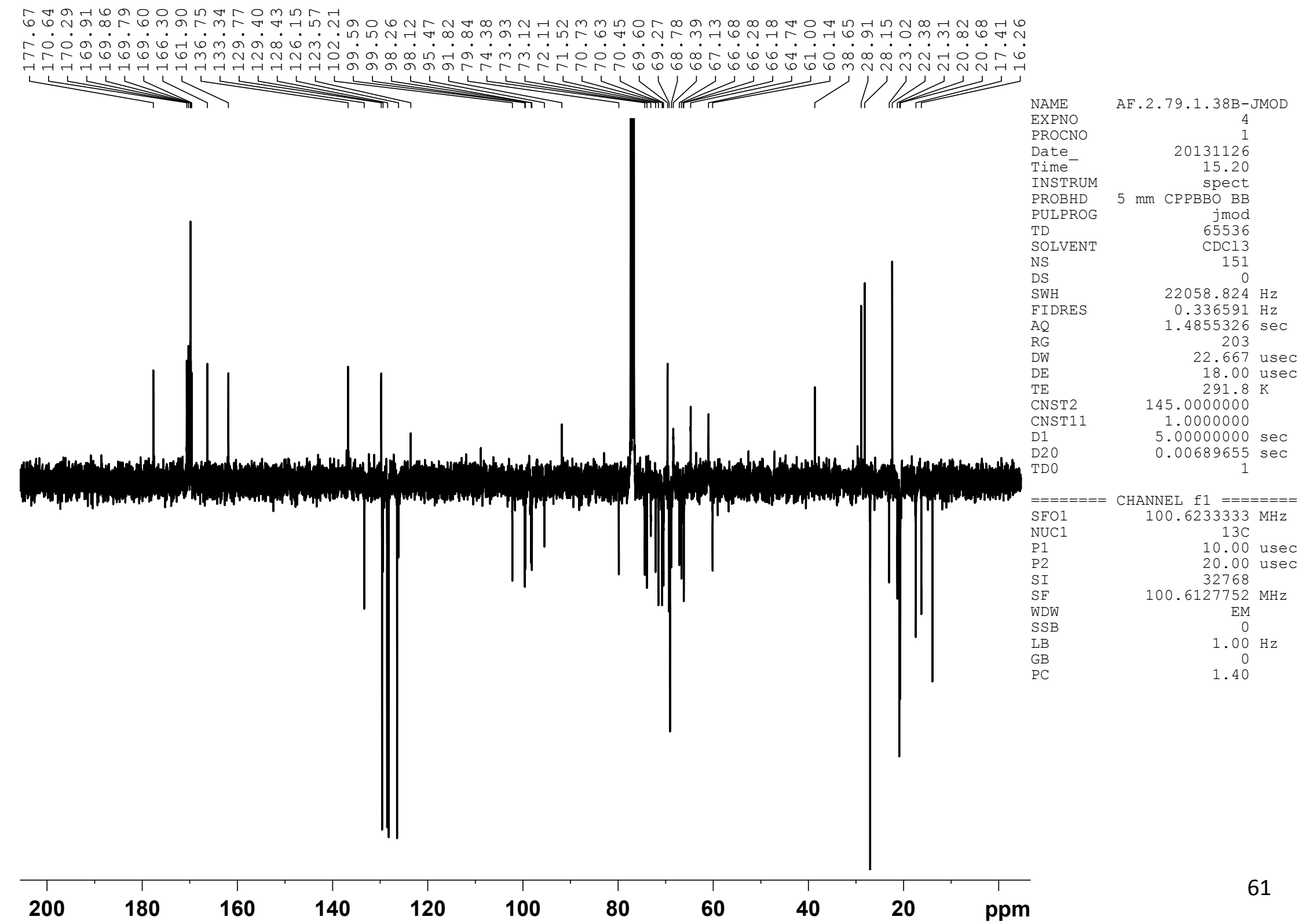


Compound 23, edited $\mathrm{HSQC}\left(\mathrm{CH}\right.$ and $\mathrm{CH}_{3}$ blue, $\mathrm{CH}_{2}$ black), $400 \mathrm{MHz}, \mathrm{CDCl}_{3}, \sim 80 \%$ pure (see text, $20 \%$ suspected orthoester)

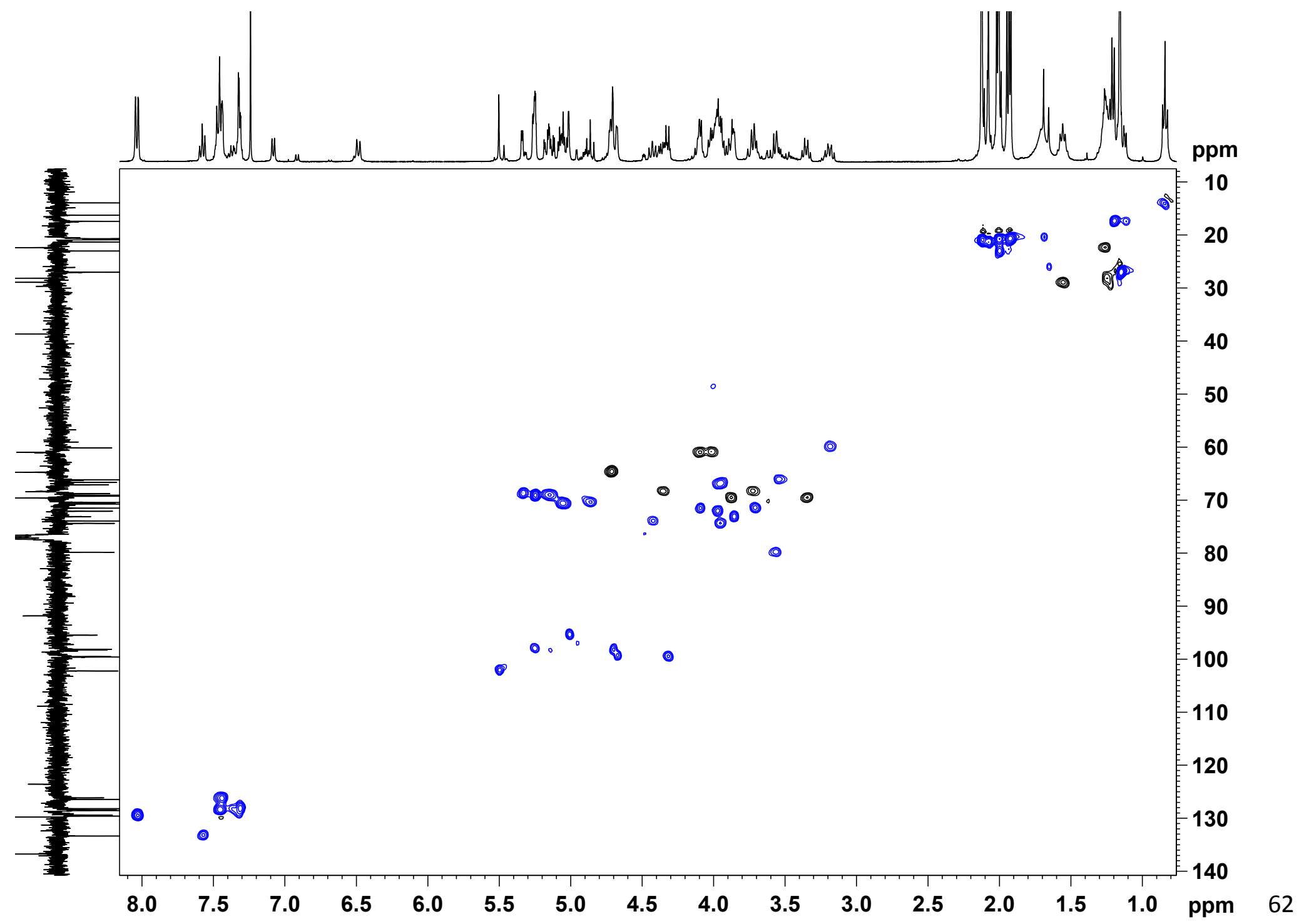


Compound 24, $600 \mathrm{MHz}, \mathrm{CDCl}_{3}$

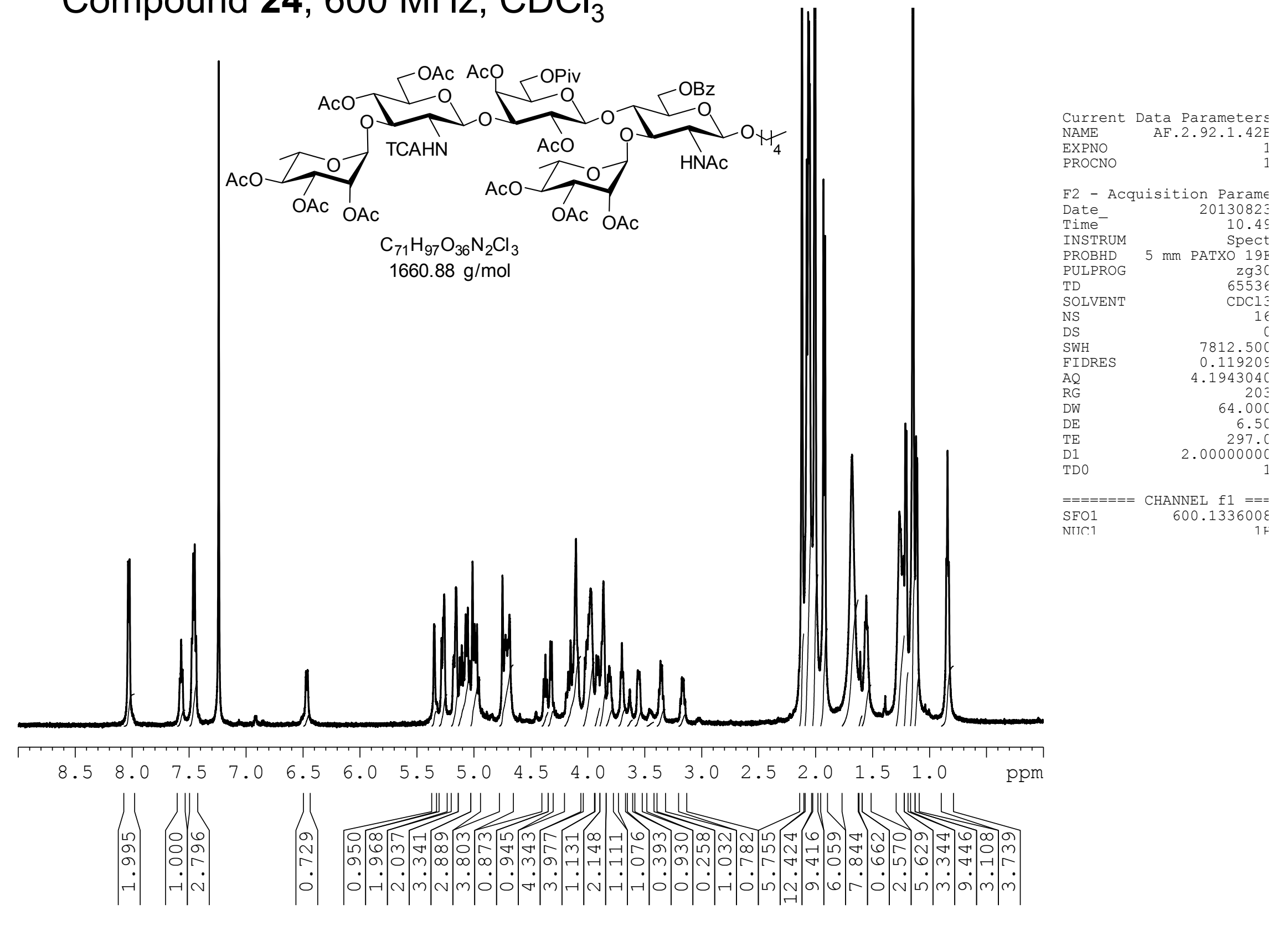


Compound 24, COSY, $600 \mathrm{MHz}, \mathrm{CDCl}_{3}$

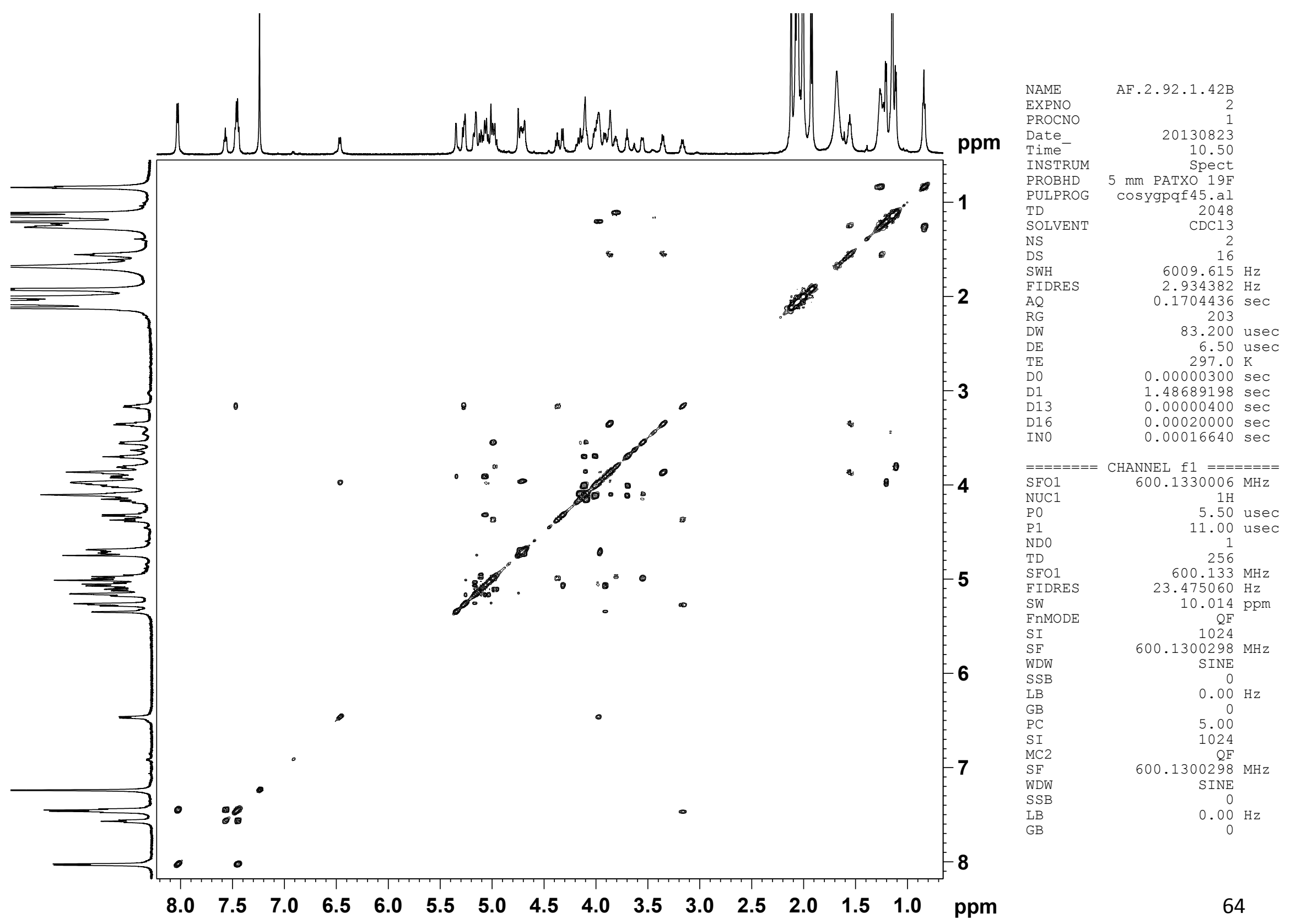




\section{Compound 24, $101 \mathrm{MHz}, \mathrm{CDCl}_{3}$}

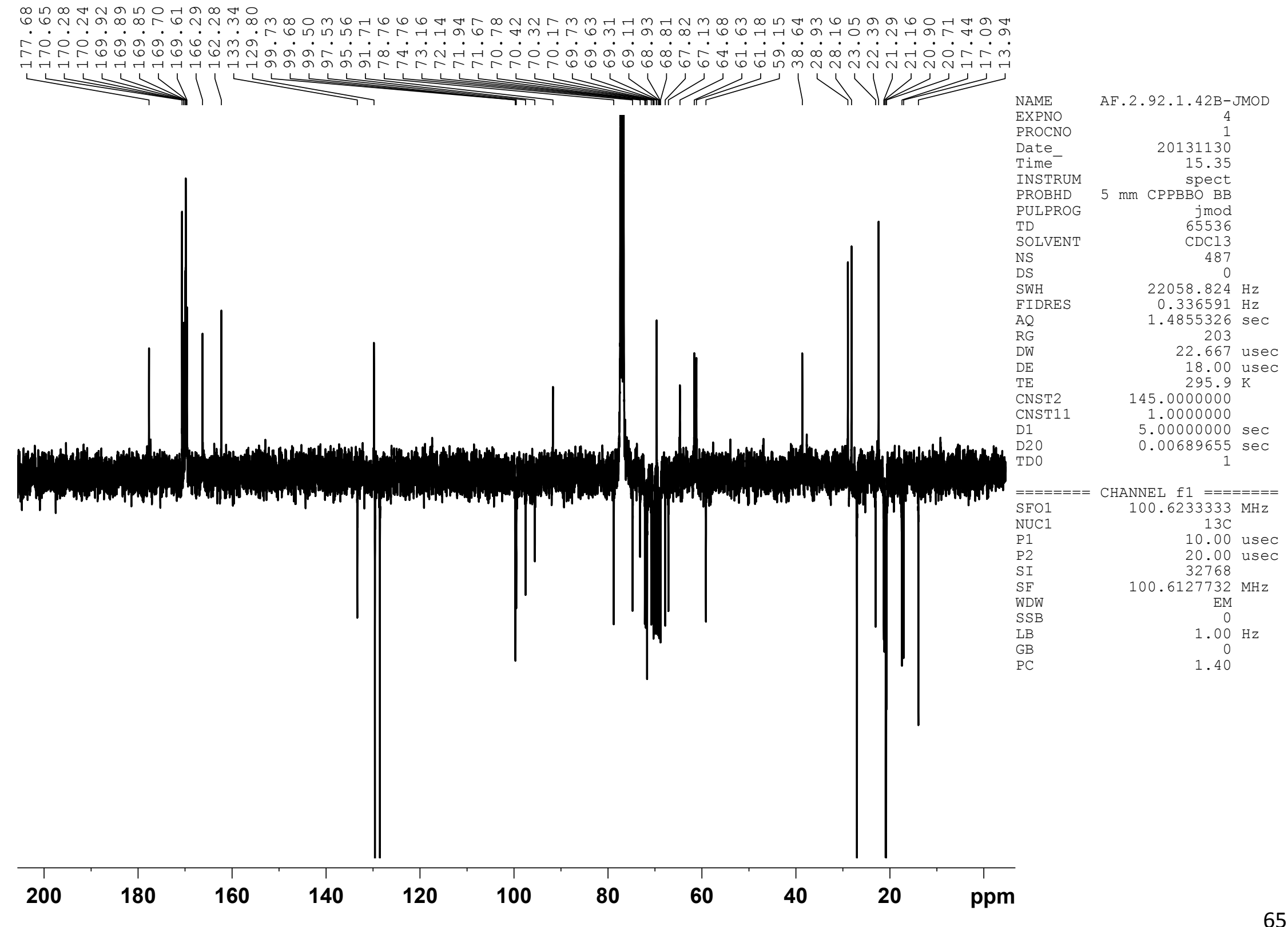


Compound 24, edited HSQC ( $\mathrm{CH}$ and $\mathrm{CH}_{3}$ blue, $\mathrm{CH}_{2}$ black), $600 \mathrm{MHz}, \mathrm{CDCl}_{3}$

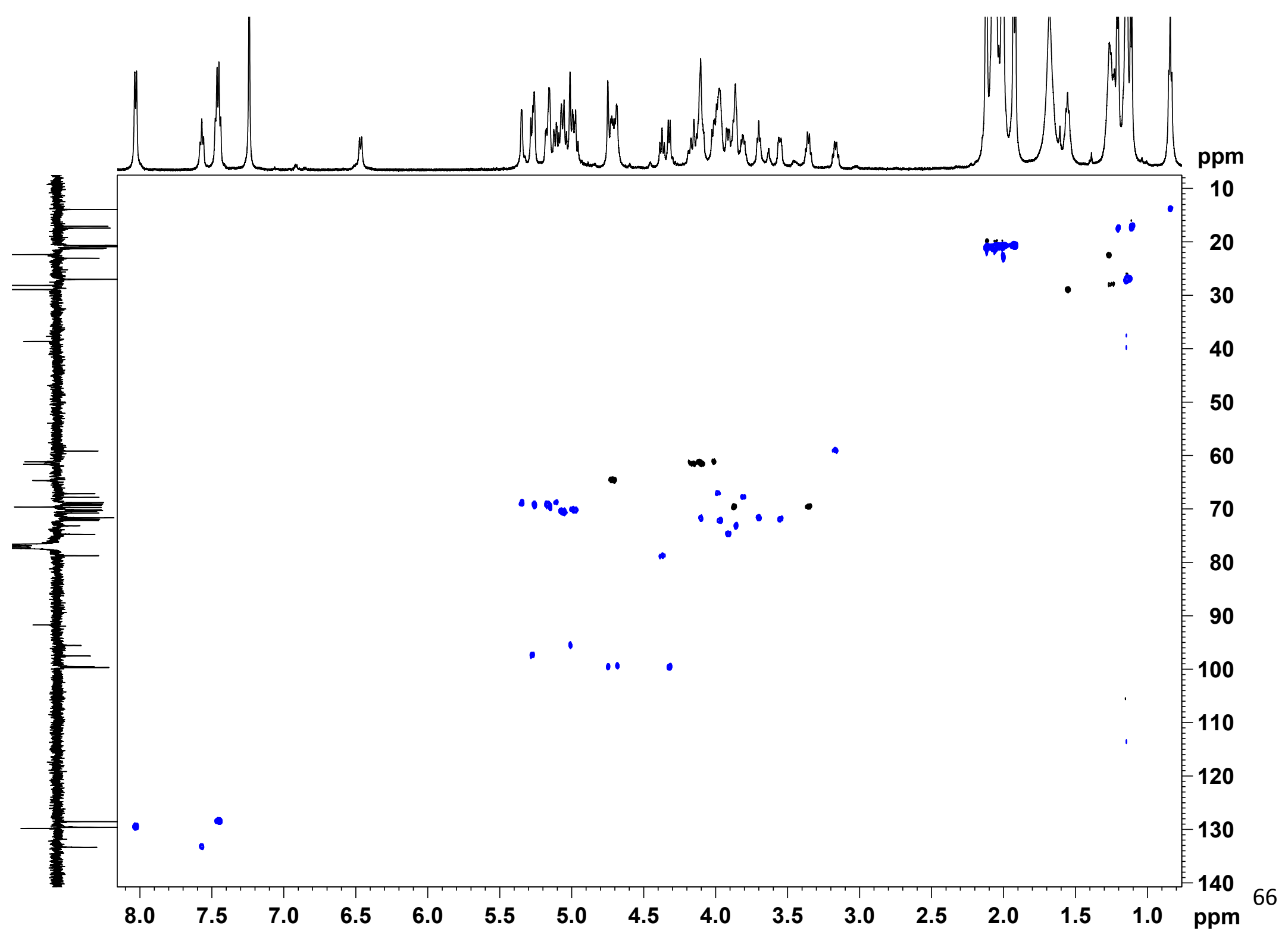




\section{Compound 26, $400 \mathrm{MHz}, \mathrm{CDCl}_{3}$}
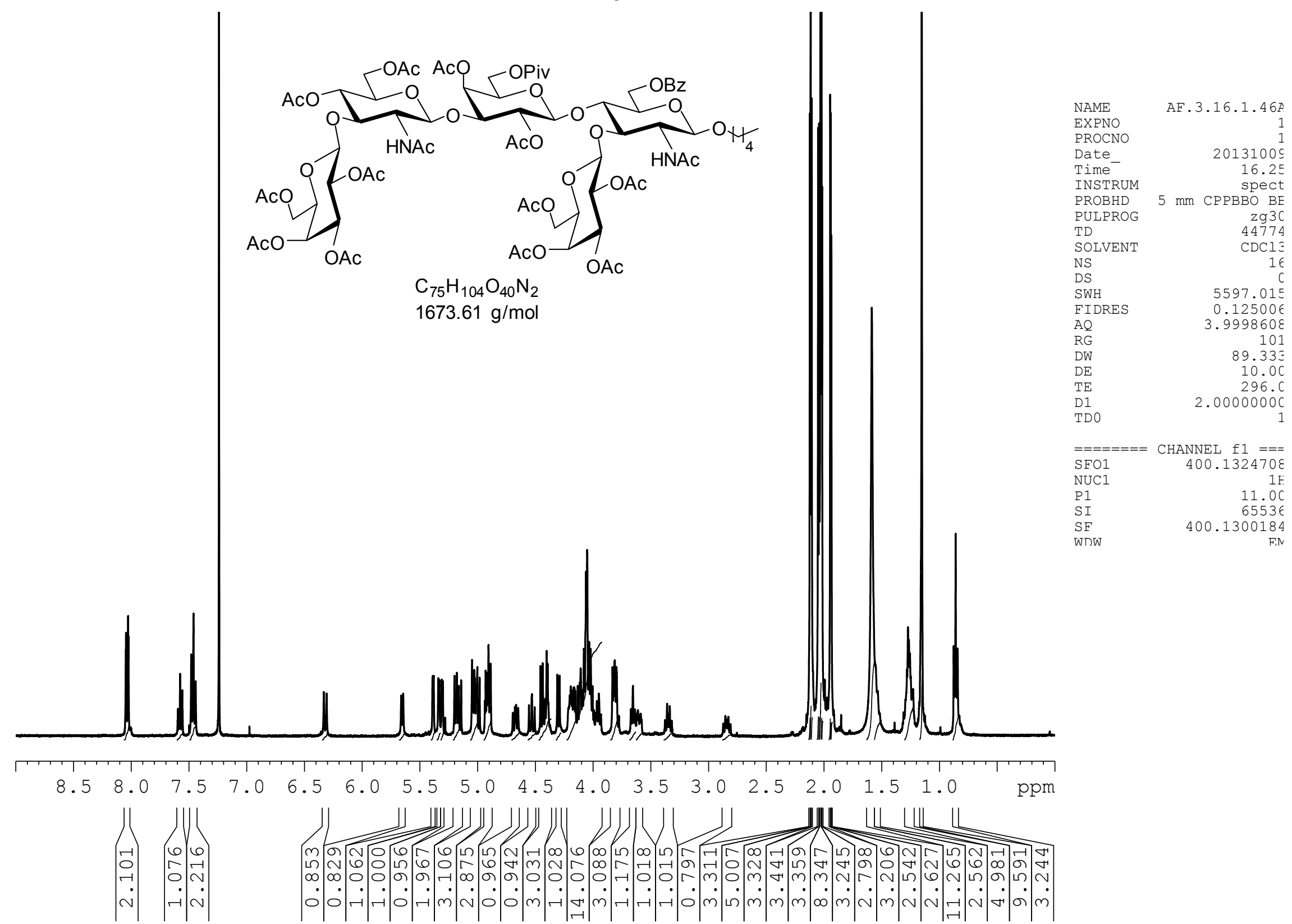
Compound 26, COSY, $400 \mathrm{MHz}, \mathrm{CDCl}_{3}$

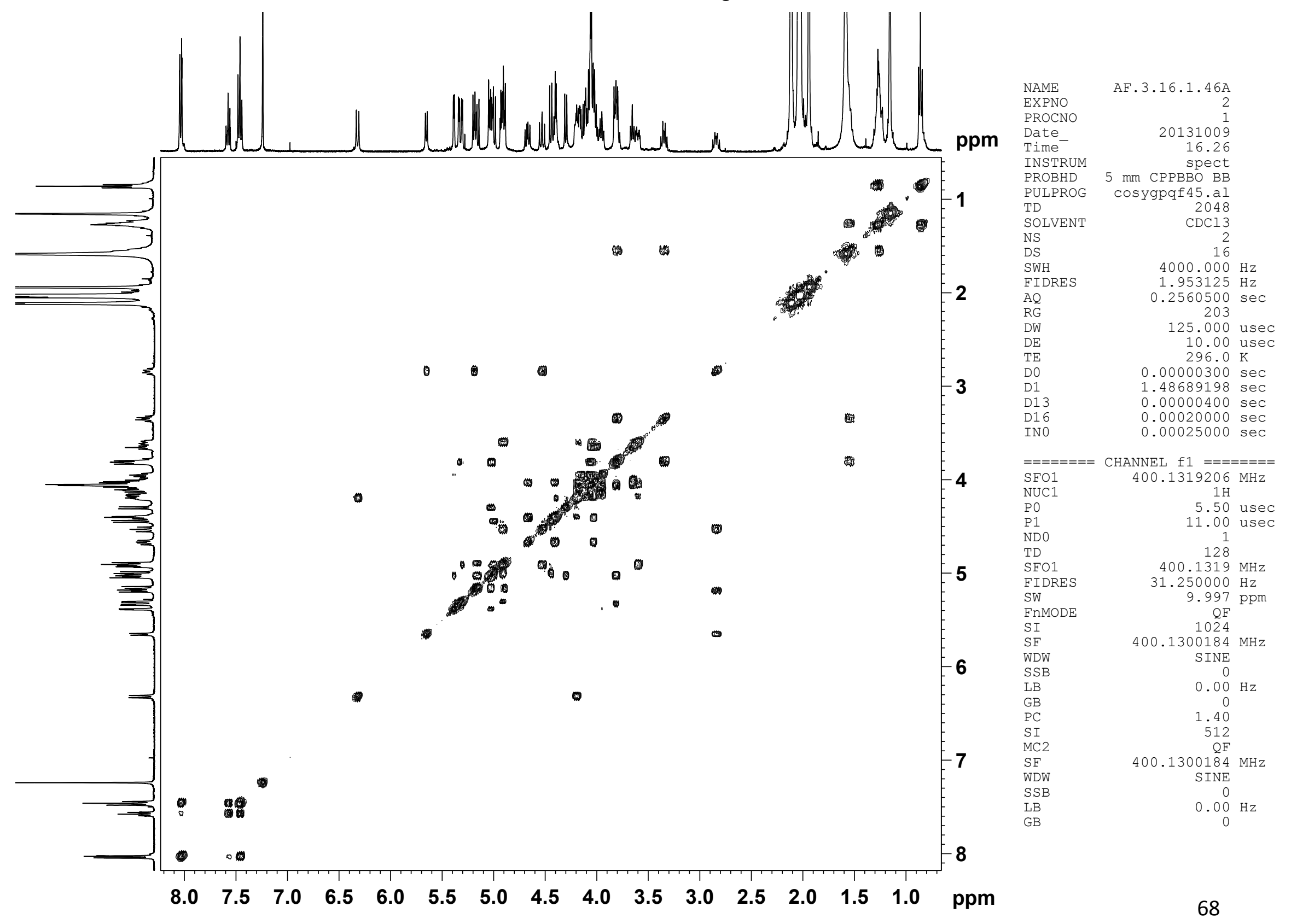




\section{Compound 26, $101 \mathrm{MHz}, \mathrm{CDCl}_{3}$}

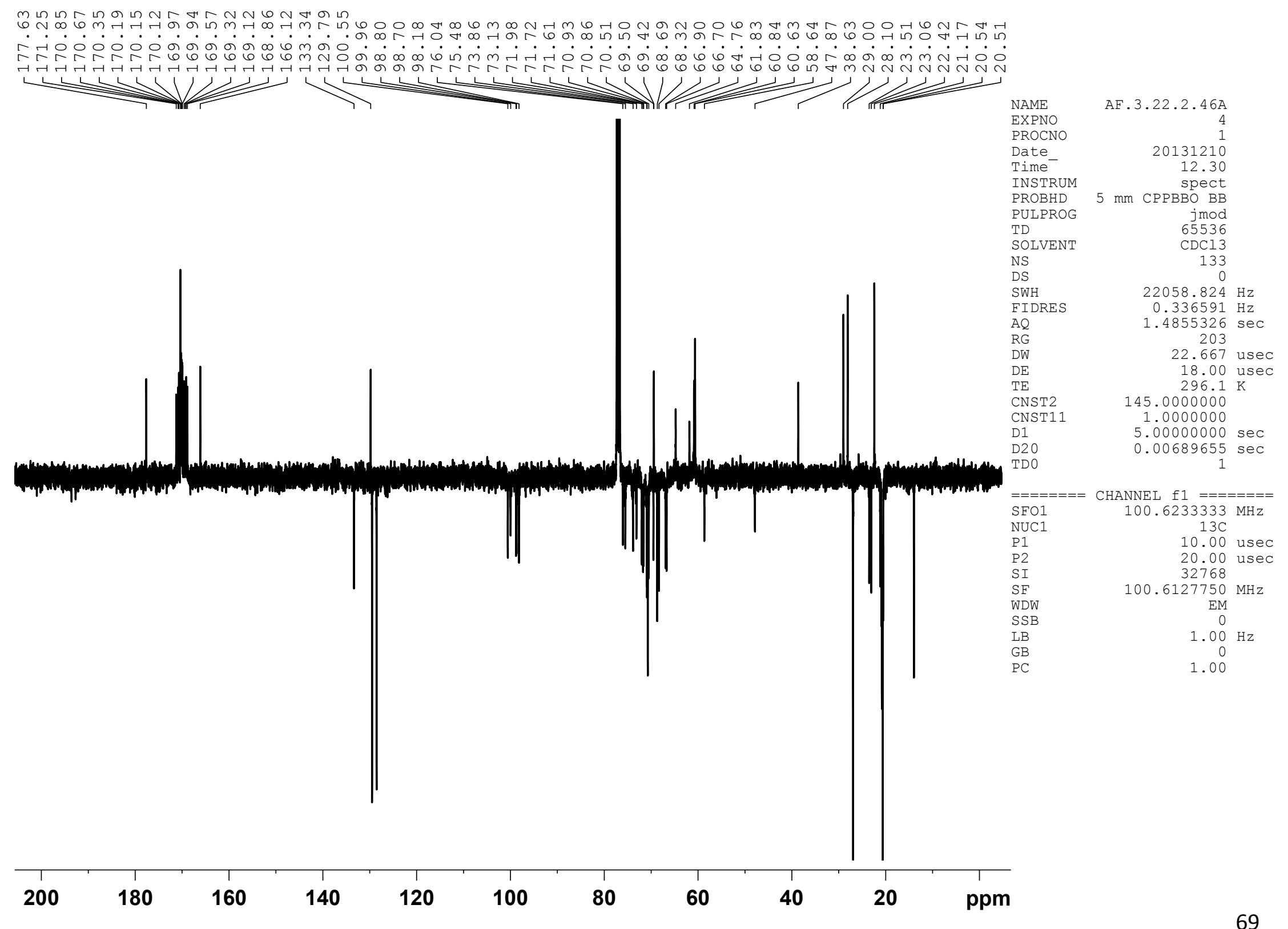


Compound 26, edited HSQC ( $\mathrm{CH}$ and $\mathrm{CH}_{3}$ blue, $\mathrm{CH}_{2}$ black), $400 \mathrm{MHz}, \mathrm{CDCl}_{3}$

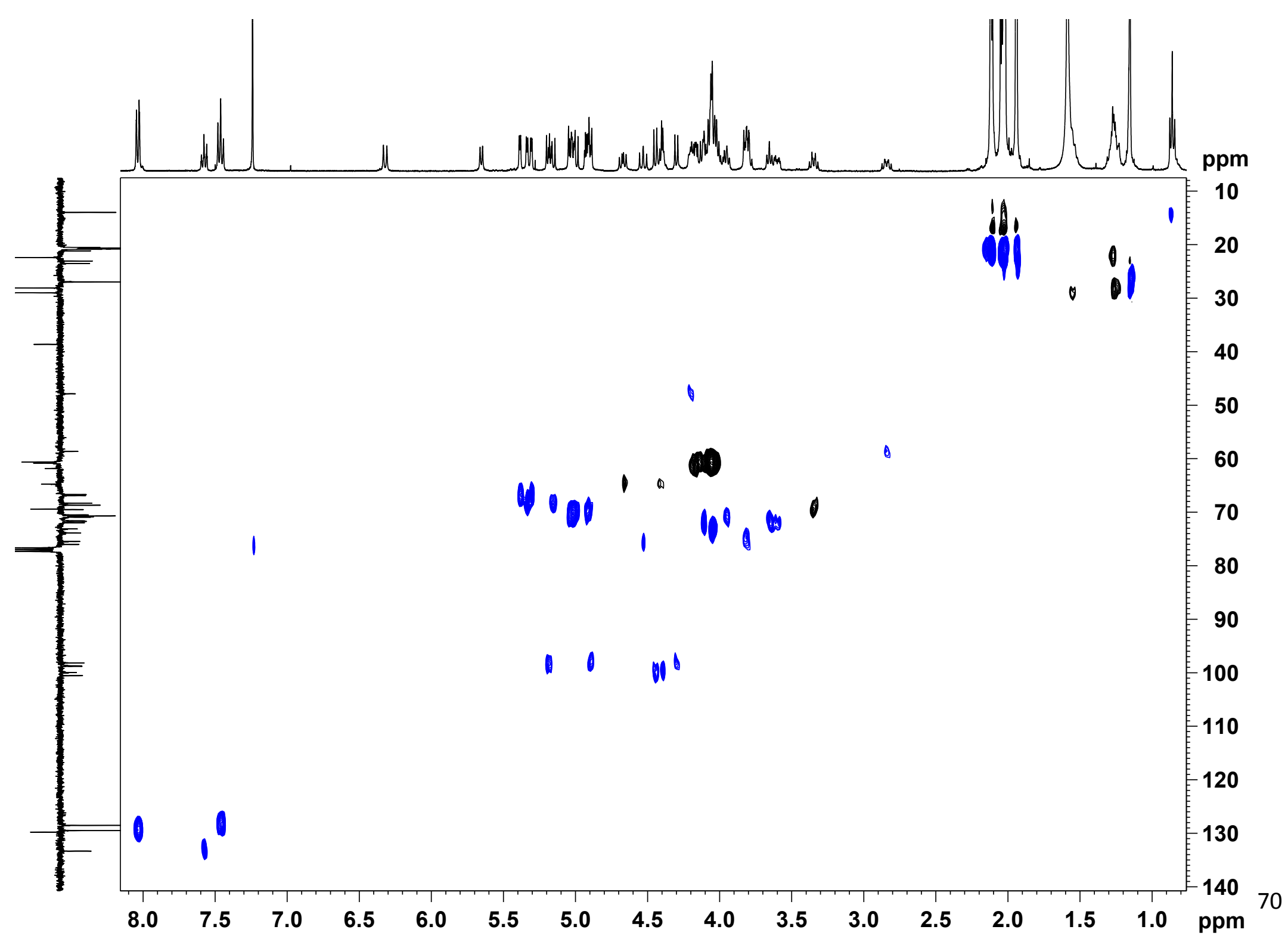


Compound 27, $600 \mathrm{MHz}, \mathrm{CDCl}_{3}$

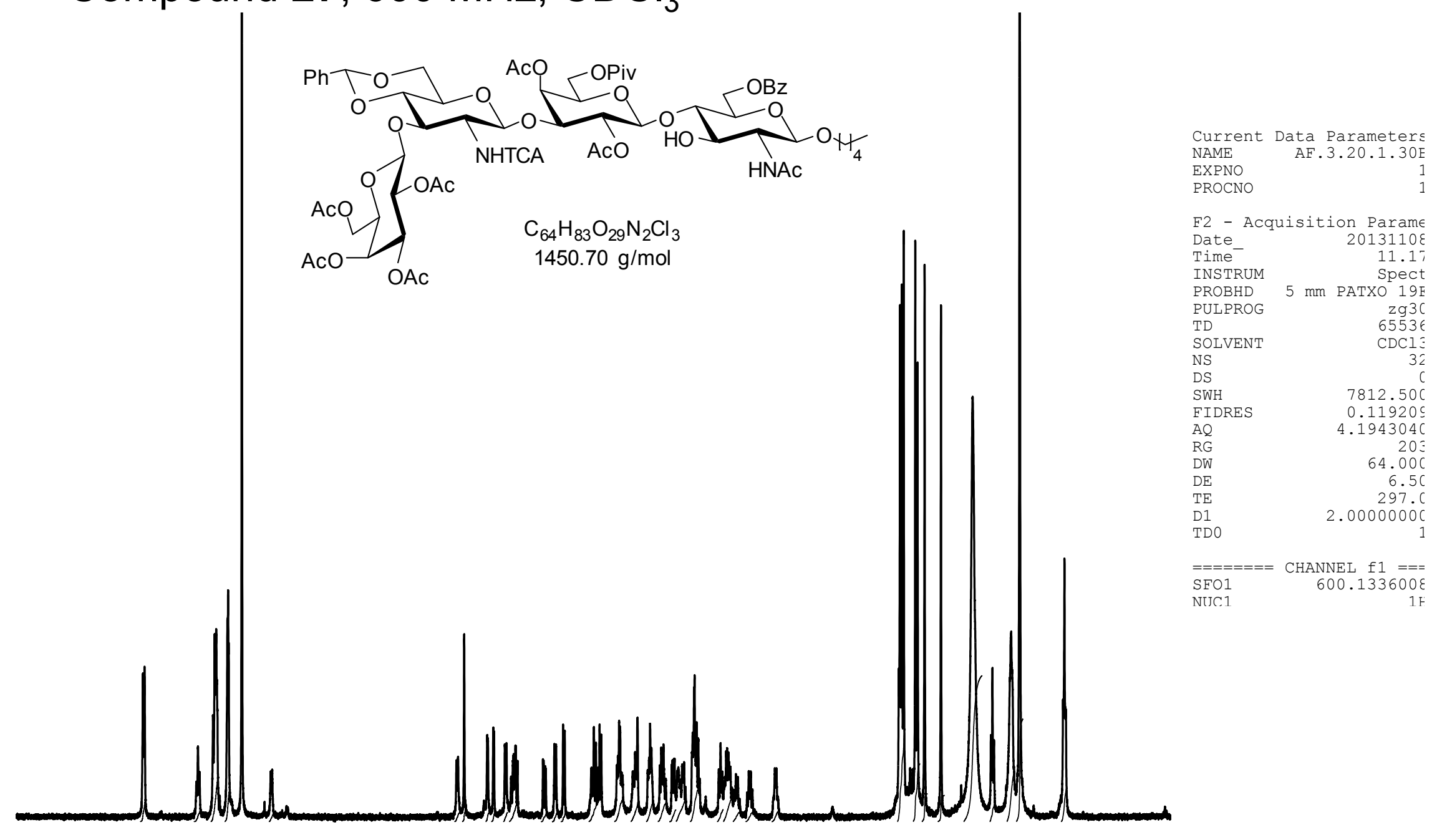

$\begin{array}{llllllllllllllllllllll}8.5 & 8.0 & 7.5 & 7.0 & 6.5 & 6.0 & 5.5 & 5.0 & 4.5 & 4.0 & 3.5 & 3.0 & 2.5 & 2.0 & 1.5 & 1.0 & \mathrm{ppm}\end{array}$

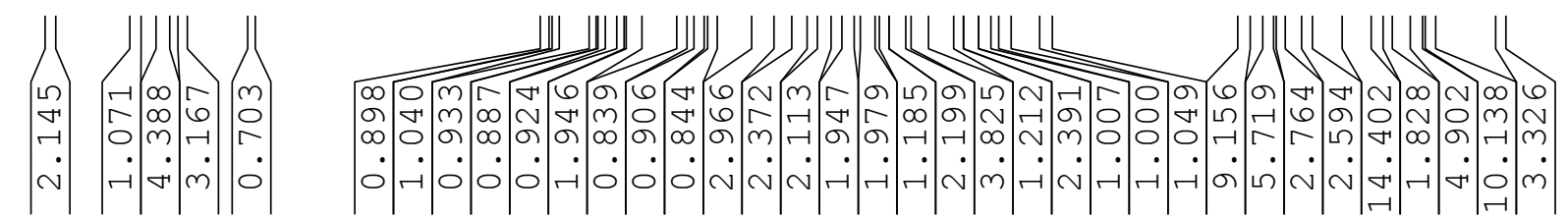


Compound 27, $600 \mathrm{MHz}, \mathrm{CDCl}_{3}$

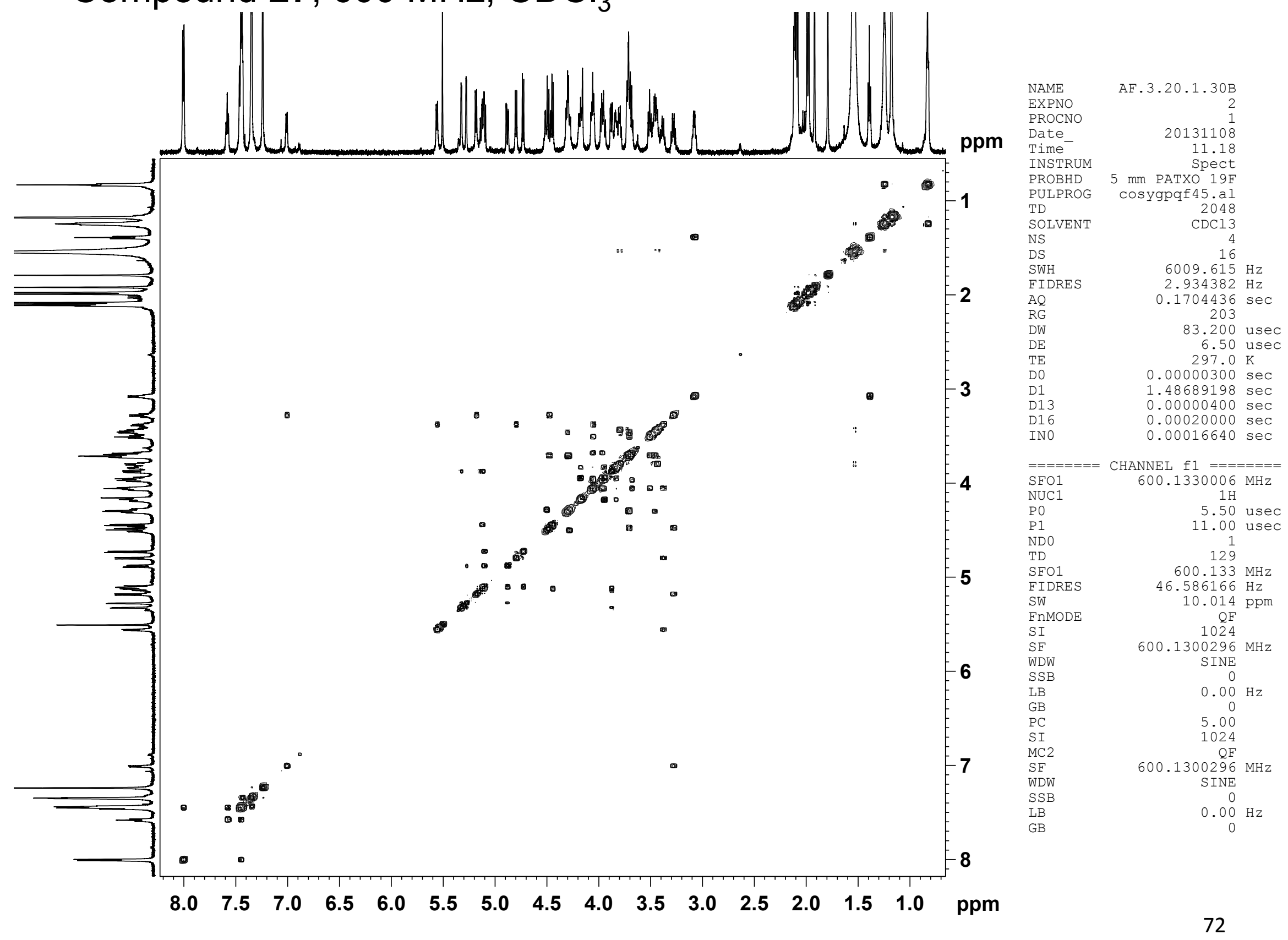




\section{Compound 27, $101 \mathrm{MHz}, \mathrm{CDCl}_{3}$}

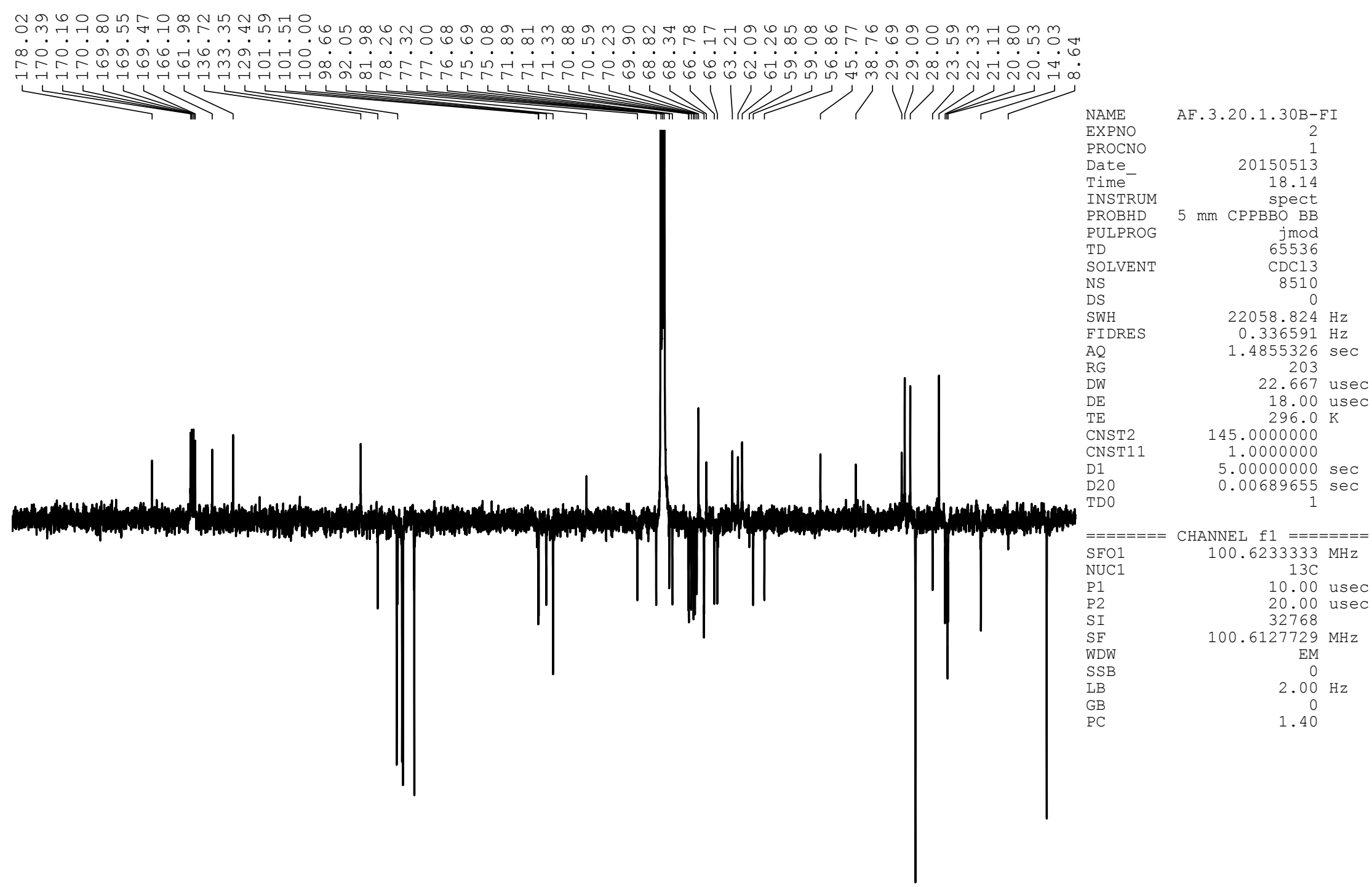

$\begin{array}{lllllllllll}200 & 180 & 160 & 140 & 120 & 100 & 80 & 60 & 40 & 20 & \mathrm{ppm}\end{array}$


Compound 27, $\mathrm{HSQC}, 600 \mathrm{MHz}, \mathrm{CDCl}_{3}$

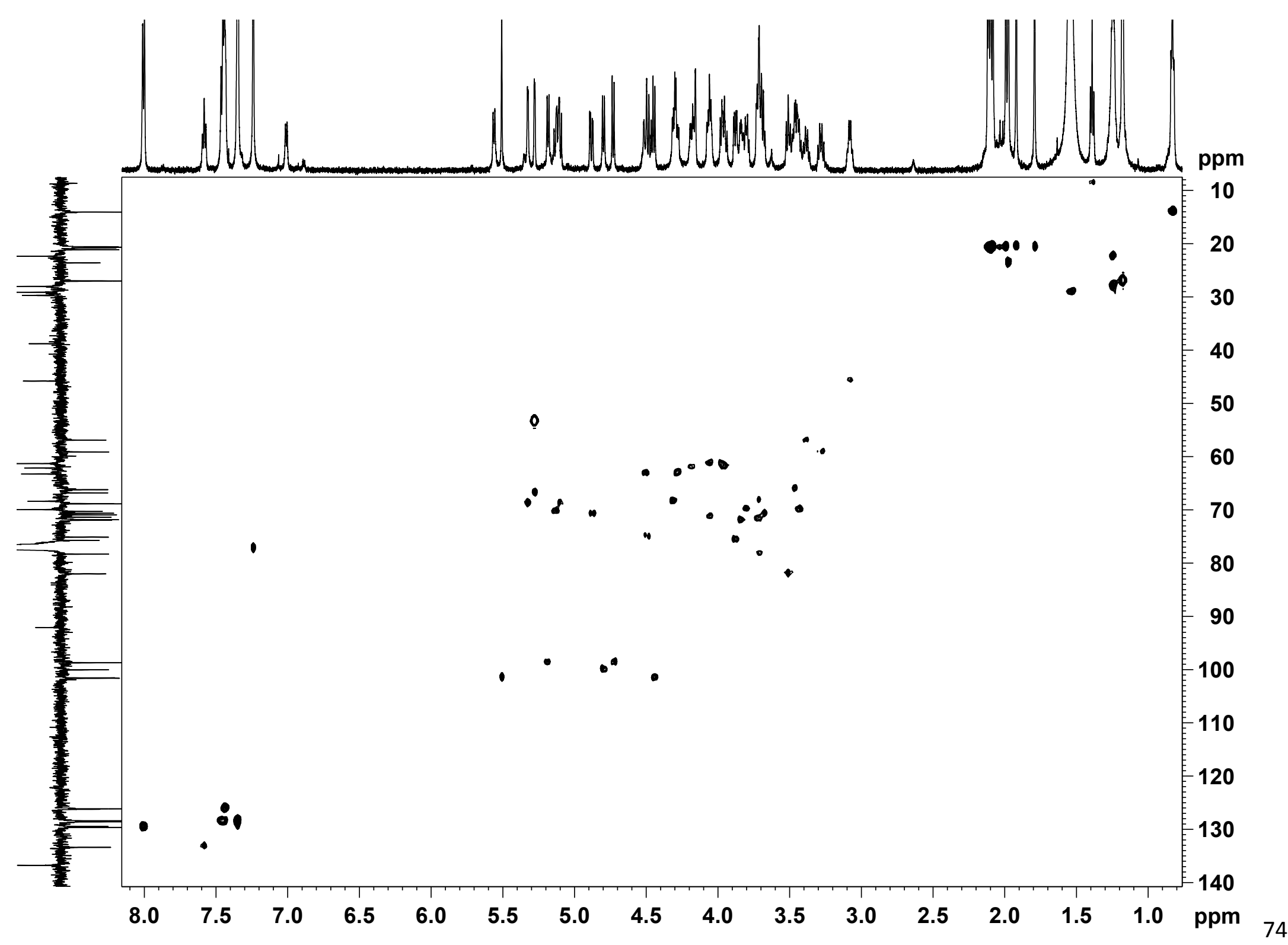


Compound 28 (major in a 75/25 mixture with compound 28), $600 \mathrm{MHz}, \mathrm{CDCl}_{3}$
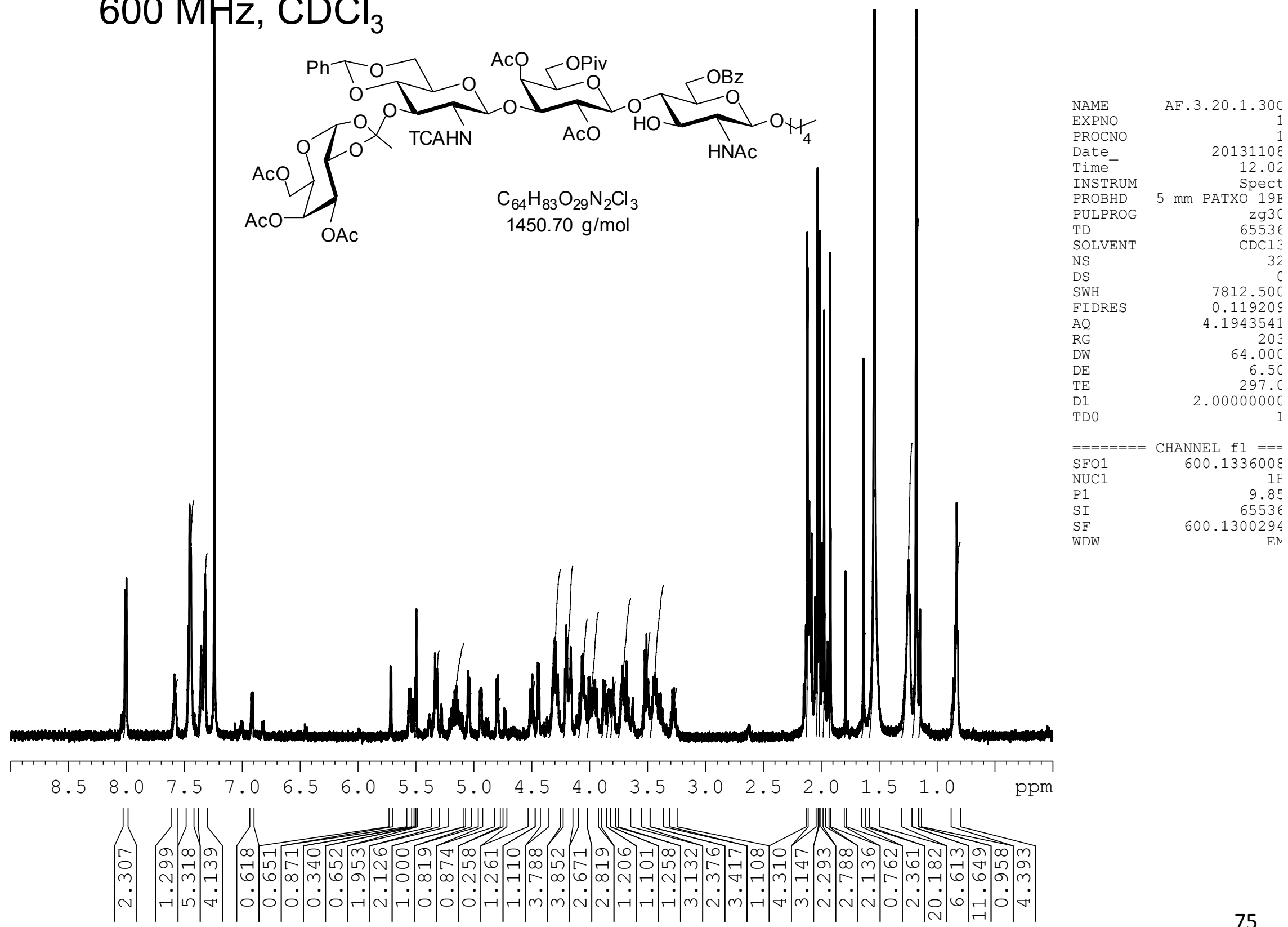
Compound 28 (major in a 75/25 mixture with compound 28), COSY, $600 \mathrm{MHz}, \mathrm{CDCl}_{3}$

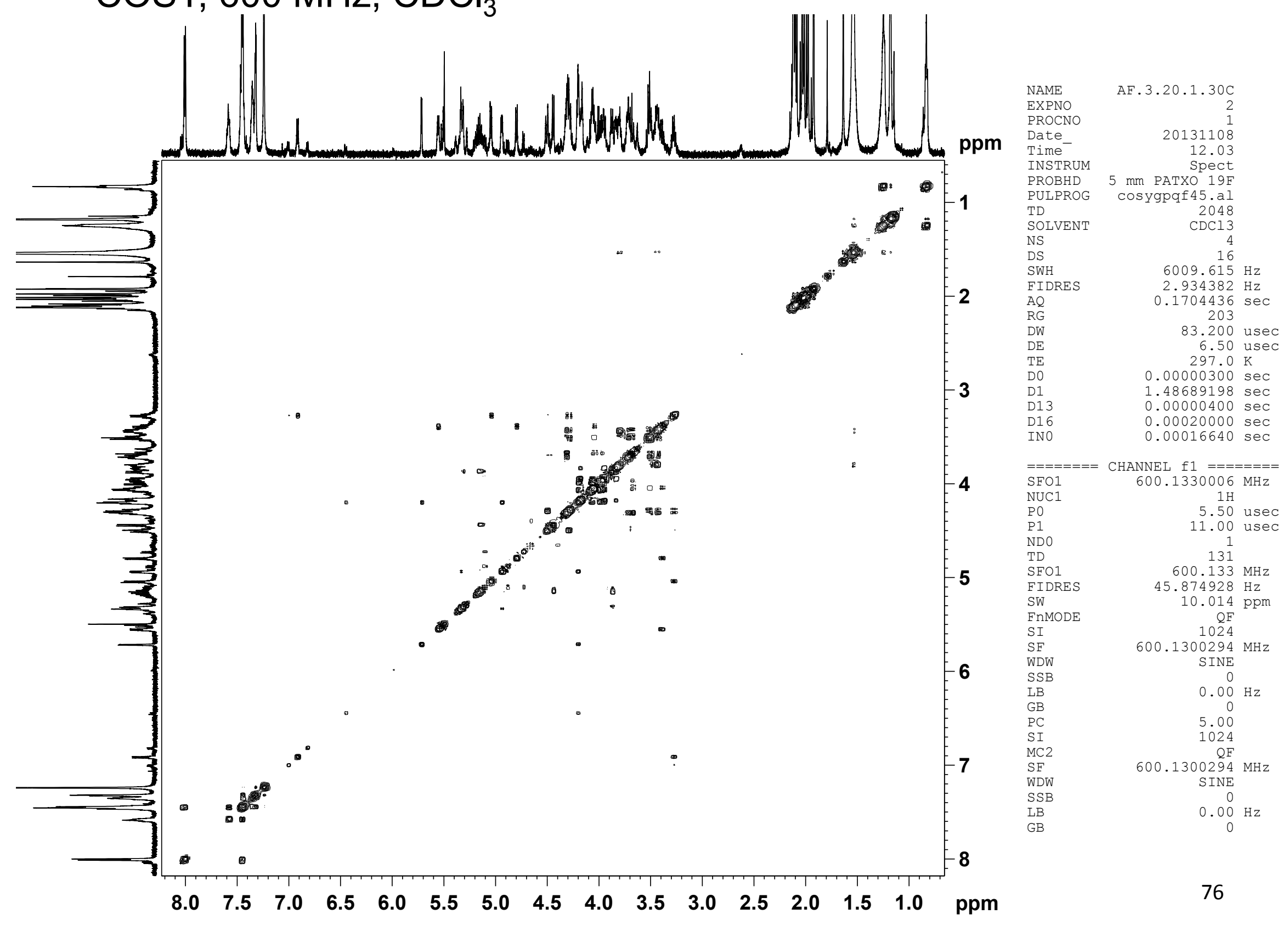


Compound 28 (major in a 75/25 mixture with compound 28), $151 \mathrm{MHz}, \mathrm{CDCl}_{3}$

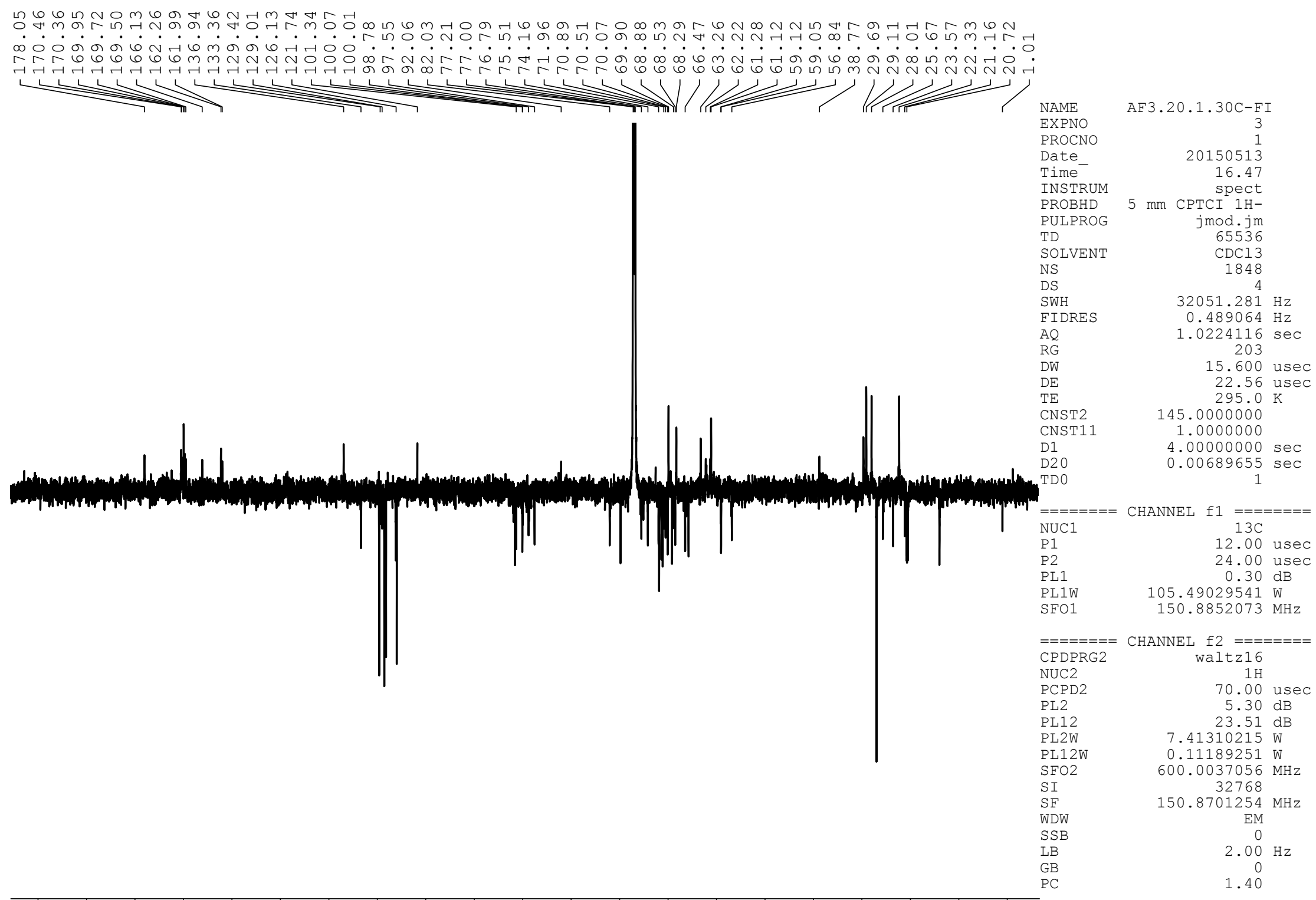


Compound 28 (major in a 75/25 mixture with compound 28), $\mathrm{HSQC}, 600 \mathrm{MHz}, \mathrm{CDCl}_{3}$

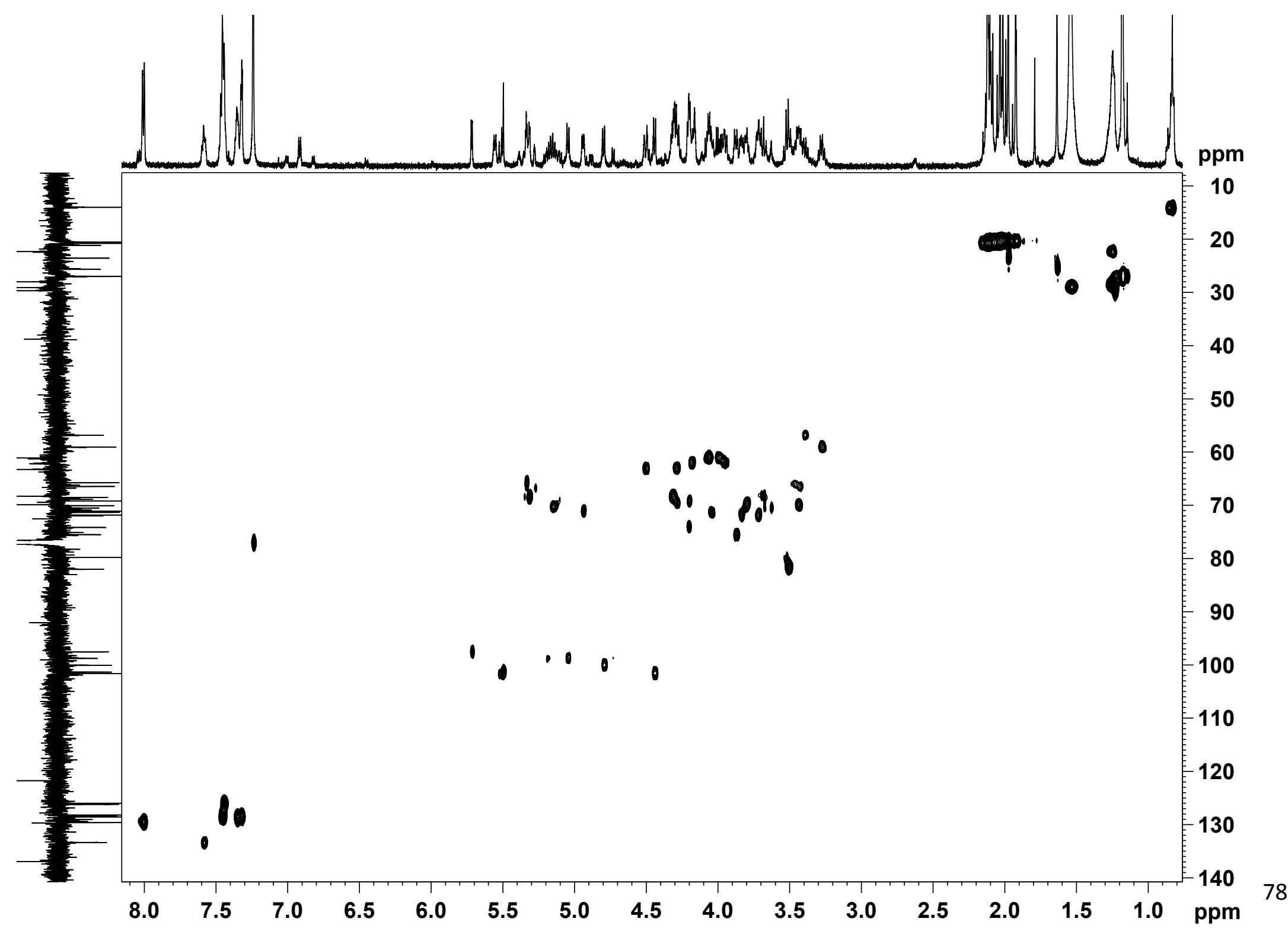


2. Scheme describing the synthesis of diol acceptors 3 and 25 via intermediate 29-31

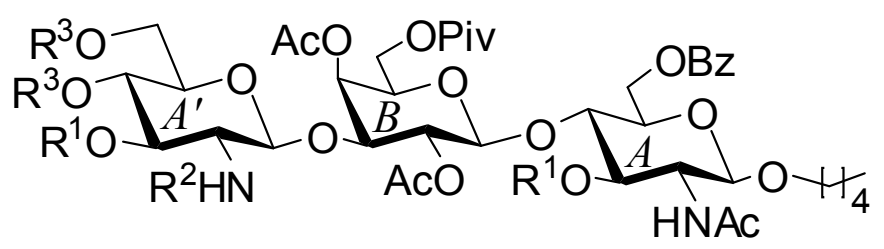

$$
\begin{aligned}
& -29 \mathrm{R}^{1}=\mathrm{CIAC}, \mathrm{R}^{2}=\mathrm{TCA}, \mathrm{R}^{3}=\mathrm{PhCH}<(75 \%) \\
& \begin{aligned}
\mathrm{AcOH} \longrightarrow 30 \mathrm{R}^{1}=\mathrm{CIAC}, \mathrm{R}^{2}=\mathrm{TCA}, \mathrm{R}^{3}=\mathrm{H} \text { (quant) } \\
\left(\mathrm{H}_{2} \mathrm{~N}\right)_{2} \mathrm{CS}\left[31 \mathrm{R}^{1}=\mathrm{CIAc}, \mathrm{R}^{2}=\mathrm{TCA}, \mathrm{R}^{3}=\mathrm{Ac}(84 \%)\right.
\end{aligned} \mathrm{Ac}_{2} \mathrm{O} \\
& \left(\mathrm{H}_{2} \mathrm{~N}\right)_{2} \mathrm{CS} \longrightarrow 3 \mathrm{R}^{1}=\mathrm{H}, \mathrm{R}^{2}=\mathrm{TCA}, \mathrm{R}^{3}=\mathrm{Ac}(89 \%) \square \mathrm{Zn}, \mathrm{AcOH} \\
& 25 \mathrm{R}^{1}=\mathrm{H}, \mathrm{R}^{2}=\mathrm{Ac}, \mathrm{R}^{3}=\mathrm{Ac}(89 \%)
\end{aligned}
$$

Synthesis of acceptor $\mathbf{3}$ and $\mathbf{2 5}$ from chloroacetylated analogue 29. ${ }^{11}$ 


\section{Experimental procedures for the synthesis of diol acceptors 3 and 25}

3.1. Pentyl O-[3-O-(chloro)acetyl-2-deoxy-2-(trichloro)acetamido- $\beta$-D-glucopyranosyl]-(1 $\rightarrow 3)-O-(2,4-d i-O-a c e t y l-6-O-p i v a l o y l-$ $\beta$-D-galactopyranosyl)-(1 $\rightarrow 4$ )-2-acetamido-6-O-benzoyl-3-O-(chloro)acetyl-2-deoxy- $\beta$-D-glucopyranoside (30)

Trisaccharide $29^{11}$ (300 mg, $0.24 \mathrm{mmol}$ ) was dissolved in 90\% aq AcOH (30 mL), the solution was stirred at $80{ }^{\circ} \mathrm{C}$ for 2 $\mathrm{h}$, then co-concentrated with toluene $(2 \times 100 \mathrm{~mL})$. Pure diol $30(279 \mathrm{mg}$, quant $)$ was obtained as a brownish powder. $[\alpha]_{\mathrm{D}}-2.0(c$ 1.0, MeOH). ${ }^{1} \mathrm{H}$ NMR ( $\left.\mathrm{CD}_{3} \mathrm{OD}, 400 \mathrm{MHz}\right): \delta_{\mathrm{H}} 8.23(\mathrm{~d}, J=9.3 \mathrm{~Hz}, 1 \mathrm{H}, \mathrm{NH}), 8.03-7.52(3 \mathrm{~m}, 5 \mathrm{H}, \mathrm{Ar}), 5.43(\mathrm{~d}, J=3.3 \mathrm{~Hz}, 1 \mathrm{H}$, H-4'), 5.25 (dd, $J=9.0,10.7$ Hz, 1 H, H-3’), 5.18 (dd, $J=8.5,10.6$ Hz, 1 H, H-3), 4.97 (dd, J = 8.0, 10.1 Hz, 1 H, H-2’), 4.84 (d, $J=8.2 \mathrm{~Hz}, 1 \mathrm{H}, \mathrm{H}-1$ ”), 4.63 (dd, $J=1.8,12.2 \mathrm{~Hz}, 1 \mathrm{H}, \mathrm{H}-6 \mathrm{a}), 4.55$ (d, $J=8.4 \mathrm{~Hz}, 1 \mathrm{H}, \mathrm{H}-1$ ), 4.48 (d, J= 8.0 Hz, 1 H, H-1'), 4.40 (dd, $J=4.5,12.2 \mathrm{~Hz}, 1 \mathrm{H}, \mathrm{H}-6 \mathrm{~b}), 4.25-4.18$ (m, $\left.3 \mathrm{H}, \mathrm{COCH}{ }_{2} \mathrm{Cl}, \mathrm{COCH} H \mathrm{Cl}\right), 4.13-4.06$ (m, $3 \mathrm{H}, \mathrm{H}-6 \mathrm{ab}$ ', $\left.\mathrm{COCH} H \mathrm{Cl}\right), 4.02$ (dd, $J$ = 3.5, 10.2 Hz, 1 H, H-3'), 3.90-3.83 (m, 3 H, H-2, H-5', H-6a"), 3.82-3.76 (m, 3 H, H-4, H-5, OCH $\mathrm{HCH}_{2}$ ), $3.67-3.60$ (m, 2 H, H-2", H-6b”), 3.50 (dd, J = 9.8, 18.8 Hz, 1 H, H-4”), 3.46 (m, 1 H, OCH HCH ${ }_{2}$ ), 3.36 (m, 1 H, H-5”), 2.14, $2.09,1.89$ (3 s, 9 H, 3 $\left.\times \mathrm{COCH}_{3}\right), 1.52\left(\mathrm{~m}, 2 \mathrm{H}, \mathrm{OCH}_{2} \mathrm{CH}_{2}\right), 1.30-1.25\left(\mathrm{~m}, 4 \mathrm{H}, \mathrm{CH}_{2} \mathrm{CH}_{2} \mathrm{CH}_{3}\right), 1.23\left(\mathrm{~s}, 9 \mathrm{H}, \mathrm{C}\left(\mathrm{CH}_{3}\right)_{3}\right), 0.85(\mathrm{t}, J=6.9 \mathrm{~Hz}, 3 \mathrm{H}$, $\left.\mathrm{CH}_{2} \mathrm{CH}_{3}\right) .{ }^{13} \mathrm{C} \mathrm{NMR}\left(\mathrm{CD}_{3} \mathrm{OD}, 101 \mathrm{MHz}\right): \delta_{\mathrm{C}} 179.3,173.4,172.5,171.0,168.6,168.5,167.3,164.0(\mathrm{C}=\mathrm{O}), 134.7,131.0,130.6$, 129.9 (Ar), 102.3 (C-1'), 102.2 (C-1), 100.9 (C-1”), 77.8 (C-5”), 77.5 (C-3’), 77.4 (C-3”), 77.1 (C-4), 75.8 (C-3), 73.8 (C-5), 72.6 (C-5'), 71.8 (C-2'), $71.0\left(\mathrm{OCH}_{2} \mathrm{CH}_{2}\right), 70.8$ (C-4'), 69.3 (C-4”), 63.8 (C-6), 63.1 (C-6'), 62.1 (C-6”), 57.6 (C-2”), 55.1 (C-2), 41.9 ,

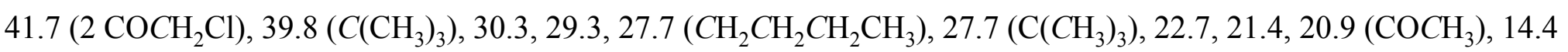
$\left(\mathrm{CH}_{2} \mathrm{CH}_{3}\right)$. HRESIMS (m/z): $[\mathrm{M}+\mathrm{H}]^{+}$calcd for $\mathrm{C}_{47} \mathrm{H}_{64} \mathrm{O}_{22} \mathrm{~N}_{2} \mathrm{Cl}_{5}$ 1183.2351, found 1183.2271. 
2.2. Pentyl O-[4,6-di-O-acetyl-3-O-(chloro)acetyl-2-deoxy-2-(trichloro)acetamido- $\beta$-D-glucopyranosyl]-(1 $\rightarrow 3)-\mathrm{O}-(2,4-d i-\mathrm{O}-$ acetyl-6-O-pivaloyl- $\beta$-D-galactopyranosyl)-(1 $\rightarrow 4)$-2-acetamido-6-O-benzoyl-3-O-(chloro)acetyl-2-deoxy- $\beta$-D-glucopyranoside (31)

Diol 30 (224 mg, $0.19 \mathrm{mmol})$ was dissolved in a 1:1 mixture of pyridine $/ \mathrm{Ac}_{2} \mathrm{O}(20 \mathrm{~mL})$. The solution was stirred at $\mathrm{rt}$ for $1 \mathrm{~h}$, coconcentrated with toluene $(2 \times 100 \mathrm{~mL})$ and the residue dissolved in $\mathrm{CH}_{2} \mathrm{Cl}_{2}(100 \mathrm{~mL})$ was washed with $2 \mathrm{M} \mathrm{HCl}(100 \mathrm{~mL})$. The aq layer was re-extracted with $\mathrm{CH}_{2} \mathrm{Cl}_{2}(3 \times 20 \mathrm{~mL})$, and the combined organic layers were dried and concentrated giving pure

diacetate $31(237 \mathrm{mg}, 99 \%)$ as a yellow amorphous solid. $[\alpha]_{\mathrm{D}}+9.8\left(c 1.0, \mathrm{CHCl}_{3}\right) .{ }^{1} \mathrm{H} \mathrm{NMR}\left(\mathrm{CDCl}_{3}, 400 \mathrm{MHz}\right): \delta_{\mathrm{H}} 7.99-7.47(3$ m, $5 \mathrm{H}, \mathrm{Ar}), 6.87$ (d, $J=8.0 \mathrm{~Hz}, 1 \mathrm{H}, \mathrm{NH}$ ), 5.71 (d, $J=9.1 \mathrm{~Hz}, 1 \mathrm{H}, \mathrm{NH}), 5.46$ (dd, $J=9.2,10.7 \mathrm{~Hz}, 1 \mathrm{H}, \mathrm{H}-3$ ”), 5.30 (d, $J=3.4$ Hz, 1 H, H-4'), 5.18 (dd, J=8.1, 9.6 Hz, 1 H, H-3), 5.07 (t, J=10.0 Hz, 1 H, H-4”), 5.00 (dd, J = 8.0, 9.9 Hz, 1 H, H-2'), 4.94 (d, $J=8.0 \mathrm{~Hz}, 1 \mathrm{H}, \mathrm{H}-1$ ”), 4.67 (dd, $J=2.6,12.0 \mathrm{~Hz}, 1 \mathrm{H}, \mathrm{H}-6 \mathrm{a}), 4.53$ (d, J=7.4 Hz, 1 H, H-1), 4.36 (dd, $J=4.7,11.9$ Hz, 1 H, H-6b), 4.33 (d, J=8.0 Hz, 1 H, H-1'), 4.23 (dd, J=2.4, 12.4 Hz, 1 H, H-6a”), 4.16-3.91 (m, 8 H, H-2, H-6ab', H-6b”, $2 \times$ $\mathrm{COCH}_{2} \mathrm{Cl}$ ), 3.84-3.76 (m, 3 H, H-4, H-3', OCHHCH ), 3.75-3.57 (m, 4 H, H-5, H-5', H-2”, H-5”), 3.40 (m, 1 H, OCHHCH ), 2.10, 2.06, 2.05, 2.00, $1.94\left(5 \mathrm{~s}, 15 \mathrm{H}, 5 \times \mathrm{COCH}_{3}\right), 1.52\left(\mathrm{~m}, 2 \mathrm{H}, \mathrm{OCH}_{2} \mathrm{CH}_{2}\right), 1.27-1.20\left(\mathrm{~m}, 4 \mathrm{H}, \mathrm{CH}_{2} \mathrm{CH}_{2} \mathrm{CH}_{3}\right), 1.17\left(\mathrm{~s}, 9 \mathrm{H}_{\text {, }}\right.$ $\left.\mathrm{C}\left(\mathrm{CH}_{3}\right)_{3}\right), 0.83\left(\mathrm{t}, J=6.8 \mathrm{~Hz}, 3 \mathrm{H}, \mathrm{CH}_{2} \mathrm{CH}_{3}\right) .{ }^{13} \mathrm{C} \mathrm{NMR}\left(\mathrm{CDCl}_{3}, 101 \mathrm{MHz}\right): \delta_{\mathrm{C}} 177.9,170.7,170.1,169.8,169.4,169.2,167.3$, 167.0, 166.0, $161.9(\mathrm{C}=\mathrm{O}), 133.6,129.5,129.4,128.7$ (Ar), 100.8 (C-1, C-1'), 99.0 (C-1”), $91.9\left(\mathrm{CCl}_{3}\right), 75.8(\mathrm{C}-3$ ') 75.0 (C-4), 73.6 (C-3), 72.5 (C-5), 72.3 (C-5”), 71.8 (C-3”), 71.4 (C-5'), 70.7 (C-2'), $70.0\left(\mathrm{OCH}_{2} \mathrm{CH}_{2}\right), 68.6$ (C-4'), 68.0 (C-4”), 62.6 (C-6),

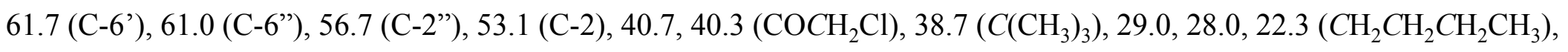
$27.1\left(\mathrm{C}_{\left.\left(\mathrm{CH}_{3}\right)_{3}\right)}\right), 23.3,21.1,20.7,20.6\left(\mathrm{COCH}_{3}\right), 14.0\left(\mathrm{CH}_{2} \mathrm{CH}_{3}\right)$. HRESIMS $(\mathrm{m} / \mathrm{z})$ : $[\mathrm{M}+\mathrm{H}]^{+}$calcd for $\mathrm{C}_{51} \mathrm{H}_{68} \mathrm{O}_{24} \mathrm{~N}_{2} \mathrm{Cl}_{5} \mathrm{H}$ 1267.2605, found 1267.2572. 
2.3. Pentyl O-[4,6-di-O-acetyl-2-deoxy-2-(trichloro)acetamido- $\beta$-D-glucopyranosyl]-(1 $\rightarrow 3)-\mathrm{O}-(2,4-d i-\mathrm{O}-$ acetyl-6-O-pivaloyl- $\beta$-Dgalactopyranosyl)-(l $\rightarrow 4$ )-2-acetamido-6-O-benzoyl-2-deoxy- $\beta$-D-glucopyranoside (3)

Diacetate 31 (236 mg, $0.19 \mathrm{mmol})$ and thiourea $(142 \mathrm{mg}, 1.86 \mathrm{mmol}, 10$ equiv) were dissolved in a mixture of 1:1 pyridine/EtOH (24 $\mathrm{mL}$ ). The solution was stirred at $70{ }^{\circ} \mathrm{C}$ for $18 \mathrm{~h}$, cooled to rt, diluted with $\mathrm{CHCl}_{3}(100 \mathrm{~mL})$, and successively washed with $2 \mathrm{M} \mathrm{HCl}$ $(100 \mathrm{~mL})$ and satd aq $\mathrm{NaHCO}_{3}(100 \mathrm{~mL})$. The aq layers were re-extracted with $\mathrm{CHCl}_{3}(3 \times 20 \mathrm{~mL}$ each $)$, and the combined organic layers were dried and concentrated. Column chromatography $\left(\mathrm{CH}_{2} \mathrm{Cl}_{2} / \mathrm{MeOH}, 20: 1\right)$ gave diacetate acceptor 3 (248 $\left.\mathrm{mg}, 89 \%\right)$ pure as a yellow amorphous solid. $[\alpha]_{\mathrm{D}}+15.6\left(c 0.50, \mathrm{CHCl}_{3}\right) .{ }^{1} \mathrm{H} \mathrm{NMR}\left(\mathrm{CDCl}_{3}, 400 \mathrm{MHz}\right): \delta_{\mathrm{H}} 8.00-7.45(3 \mathrm{~m}, 5 \mathrm{H}, \mathrm{Ar}), 7.19(\mathrm{~d}, J=7.0 \mathrm{~Hz}$, $\left.1 \mathrm{H}, \mathrm{N}-\mathrm{H}^{\prime}\right), 5.61$ (d, $\left.J=7.9 \mathrm{~Hz}, 1 \mathrm{H}, \mathrm{NH}\right), 5.35$ (br d, $J=3.3 \mathrm{~Hz}, 1 \mathrm{H}, \mathrm{H}-4$ '), 5.14 (dd, $\left.J=8.1,10.1 \mathrm{~Hz}, 1 \mathrm{H}, \mathrm{H}-2^{\prime}\right), 5.03$ (d, $J=8.0$ Hz, 1 H, H-1"), 4.83 (t, $J=9.2 \mathrm{~Hz}, 1 \mathrm{H}, \mathrm{H}-4$ "), 4.76 (d, $J=8.2 \mathrm{~Hz}, 1 \mathrm{H}, \mathrm{H}-1), 4.51$ (dd, $J=1.9,11.7 \mathrm{~Hz}, 1 \mathrm{H}, \mathrm{H}-6 \mathrm{a}), 4.47$ (d, $J=8.0$ Hz, 1 H, H-1'), 4.30-4.08 (m, 6 H, H-6b, H-6a', H-3', H-6ab”, OH-3), 4.04-3.93 (m, 2 H, H-3, H-6b'), 3.90-3.82 (m, 2 H, H-3', H5'), 3.79 (m, $1 \mathrm{H}, \mathrm{OCH} \mathrm{HCH}_{2}$ ), 3.70 (m, $\left.1 \mathrm{H}, \mathrm{H}-5\right), 3.60$ (m, $1 \mathrm{H}, \mathrm{H}-5$ "), 3.50 (t, J = 9.4 Hz, $\left.1 \mathrm{H}, \mathrm{H}-4\right), 3.47-3.38$ (m, 2 H, H-2, $\mathrm{OCH} H \mathrm{CH}_{2}$ ), 3.27 (m, $1 \mathrm{H}, \mathrm{H}-2$ "), 3.21 (s, $1 \mathrm{H}, \mathrm{OH}-3$ ”), 2.10, 2.08, 2.07, 2.06, $1.96\left(5 \mathrm{~s}, 15 \mathrm{H}, 5 \times \mathrm{COCH}_{3}\right), 1.52$ (m, $2 \mathrm{H}$, $\left.\mathrm{OCH}_{2} \mathrm{CH}_{2}\right), 1.28-1.22\left(\mathrm{~m}, 4 \mathrm{H}, \mathrm{CH}_{2} \mathrm{CH}_{2} \mathrm{CH}_{3}\right), 1.16\left(\mathrm{~s}, 9 \mathrm{H}, \mathrm{C}\left(\mathrm{CH}_{3}\right)_{3}\right), 0.83\left(\mathrm{t}, \mathrm{J}=6.8 \mathrm{~Hz}, 3 \mathrm{H}, \mathrm{CH}_{2} \mathrm{CH}_{3}\right) .{ }^{13} \mathrm{C} \mathrm{NMR}\left(\mathrm{CDCl}_{3}, 101\right.$ $\mathrm{MHz}): \delta_{\mathrm{C}} 178.1,170.9,170.8,170.4,169.9,169.4,166.2,162.4(\mathrm{C}=\mathrm{O}), 133.3,129.6,128.5$ (Ar), $101.5\left(\mathrm{C}-1^{\prime}\right), 100.1(\mathrm{C}-1), 98.7$ (C1”), 92.1 ( $\mathrm{CCl}_{3}$ ), 82.1 (C-4), 75.7 (C-3'), 72.0 (C-5), 71.9 (C-5”), 71.8 (C-5'), 71.5 (C-3), 71.3 (C-4”), 70.3 (C-2'), 70.0 (C-3"), 69.9 $\left(\mathrm{OCH}_{2} \mathrm{CH}_{2}\right), 69.0$ (C-4'), 63.2 (C-6), 62.5 (C-6'), 61.4 (C-6”), 59.8 (C-2”), $56.7(\mathrm{C}-2), 38.7\left(\mathrm{C}^{\prime}\left(\mathrm{CH}_{3}\right)_{3}\right), 29.1,28.0,22.3$ $\left(\mathrm{CH}_{2} \mathrm{CH}_{2} \mathrm{CH}_{2} \mathrm{CH}_{3}\right), 26.9\left(\mathrm{C}_{\left.\left(\mathrm{CH}_{3}\right)_{3}\right)}\right), 23.5,21.1,20.8,20.7\left(\mathrm{COCH}_{3}\right), 14.0\left(\mathrm{CH}_{2} \mathrm{CH}_{3}\right)$. HRESIMS $(\mathrm{m} / \mathrm{z}):[\mathrm{M}+\mathrm{H}]^{+}$calcd for $\mathrm{C}_{47} \mathrm{H}_{66} \mathrm{O}_{22} \mathrm{~N}_{2} \mathrm{Cl}_{3}$ 1115.3173, found 1115.3164 . 
2.4. Pentyl O-[2-acetamido-4,6-di-O-acetyl-2-deoxy- $\beta$-D-glucopyranosyl]-(1 $\rightarrow 3)-\mathrm{O}-(2,4-d i$-O-acetyl-6-O-pivaloyl- $\beta$ D-galactopyranosyl)-(1 $\rightarrow 4$ )-2-acetamido-6-O-benzoyl-2-deoxy- $\beta$-D-glucopyranoside (25)

Trisaccharide diol acceptor 3 (190 mg, $0.17 \mathrm{mmol})$ was dissolved in AcOH (19 mL). Activated Zn dust $(3 \times 1.11 \mathrm{~g}, 3 \times$ 100 equiv) was added in portion wise every $4 \mathrm{~h}$ while the reaction mixture was sonicated at $50{ }^{\circ} \mathrm{C}$ for $12 \mathrm{~h}$. The mixture was cooled to $\mathrm{rt}$, filtered over celite and the filtrate was washed successively with $\mathrm{CH}_{2} \mathrm{Cl}_{2}(2 \times 100 \mathrm{~mL})$ and satd aq $\mathrm{NaHCO}_{3}(200 \mathrm{~mL})$. The aq layers were re-extracted with $\mathrm{CH}_{2} \mathrm{Cl}_{2}(5 \times 50 \mathrm{~mL})$, and the combined organic layers were dried and concentrated giving acetamido acceptor $25(154 \mathrm{mg}, 89 \%)$ as a colorless solid. $[\alpha]_{\mathrm{D}}+7.4(c 0.61$, $\mathrm{MeOH}) .{ }^{1} \mathrm{H} \mathrm{NMR}\left(\mathrm{CDCl}_{3}, 600 \mathrm{MHz}\right): \delta_{\mathrm{H}} 8.01-7.45$ (3 m, $\left.5 \mathrm{H}, \mathrm{Ar}\right), 5.96$ (s, $1 \mathrm{H}, \mathrm{NH}$ '), 5.59 (d, J=7.4 Hz, $\left.1 \mathrm{H}, \mathrm{NH}\right)$, 5.34 (d, $J=3.5 \mathrm{~Hz}, 1 \mathrm{H}, \mathrm{H}-4$ '), 5.13 (dd, $J=8.1,9.9 \mathrm{~Hz}, 1 \mathrm{H}, \mathrm{H}-2$ '), 4.87 (t, $J=9.5 \mathrm{~Hz}, 1 \mathrm{H}, \mathrm{H}-4$ ”), 4.84 (d, $J=8.3$ Hz, 1 H, H-1), 4.68 (d, J=8.1 Hz, 1 H, H-1”), 4.56 (dd, J=1.2, 10.3 Hz, 1 H, H-6a), 4.51 (d, J=8.0 Hz, 1 H, H-1'), 4.28 (dd, $J=5.5,11.7 \mathrm{~Hz}, 1 \mathrm{H}, \mathrm{H}-6 \mathrm{~b}), 4.24$ (dd, $J=1.5,10.4 \mathrm{~Hz}, 1 \mathrm{H}, \mathrm{H}-6 \mathrm{a}$ "), 4.20 (br s, $1 \mathrm{H}, \mathrm{OH}-3$ ), 4.16 (dd, $J=4.4$, 11.8 Hz, 1 H, H-6a'), 4.08 (t, J=9.1 Hz, 2 H, H-3), 4.05 (dd, J=4.1, 12.4 Hz, 1 H, H-6b”), 4.02-3.95 (m, 2 H, H-6b', H-3”), 3.86 (m, 1 H, H-5'), 3.81 (m, 1 H, OCH $\mathrm{HCH}_{2}$ ), 3.77 (dd, J=3.7, 14.9 Hz, 1 H, H-3'), 3.73 (m, 1 H, H-5), 3.55 (m, $1 \mathrm{H}, \mathrm{H}-5$ "), 3.51 (dd, $J=8.3,9.3 \mathrm{~Hz}, 1 \mathrm{H}, \mathrm{H}-4), 3.46$ (m, $\left.1 \mathrm{H}, \mathrm{OCH} H \mathrm{CH}_{2}\right), 3.34$ (m, $\left.1 \mathrm{H}, \mathrm{H}-2\right), 3.06$ (m, $1 \mathrm{H}, \mathrm{H}-$ 2”), 2.12, 2.10, 2.07, 2.05, 1.99, $1.97\left(6 \mathrm{~s}, 18 \mathrm{H}, 6 \times \mathrm{COCH}_{3}\right), 1.53\left(\mathrm{~m}, 2 \mathrm{H}, \mathrm{OCH}_{2} \mathrm{CH}_{2}\right), 1.28-1.22(\mathrm{~m}, 4 \mathrm{H}$, $\left.\mathrm{CH}_{2} \mathrm{CH}_{2} \mathrm{CH}_{3}\right), 1.17\left(\mathrm{~s}, 9 \mathrm{H}, \mathrm{C}\left(\mathrm{CH}_{3}\right)_{3}\right), 0.83\left(\mathrm{t}, J=6.9 \mathrm{~Hz}, 3 \mathrm{H}, \mathrm{CH}_{2} \mathrm{CH}_{3}\right) .{ }^{13} \mathrm{C} \mathrm{NMR}\left(\mathrm{CDCl}_{3}, 151 \mathrm{MHz}\right): \delta_{\mathrm{C}} 178.1$, 172.4, 170.9, 170.5, 170.4, 169.5, 166.2 (C=O), 133.4, 129.6, 128.5 (Ar), 101.3 (C-1'), 99.9 (C-1, C-1”), 82.4 (C-4), 76.2 (C-3'), 72.0 (C-5', C-5”), 71.8 (C-5), 71.4 (C-3), 71.3 (C-3”), 71.0 (C-2', C-4”), $69.1\left(\mathrm{OCH}_{2} \mathrm{CH}_{2}\right), 69.1$ (C-4'), 63.3 (C-6), 62.3 (C-6'), 61.5 (C-6”), 59.4 (C-2”), 57.1 (C-2), $38.7\left(C\left(\mathrm{CH}_{3}\right)_{3}\right), 29.1,28.0,22.3\left(\mathrm{CH}_{2} \mathrm{CH}_{2} \mathrm{CH}_{2} \mathrm{CH}_{3}\right), 26.9$ $\left(\mathrm{C}_{\left.\left(\mathrm{CH}_{3}\right)_{3}\right)}\right)$, 23.6, 23.4, 21.0, 20.9, 20.8, $20.7\left(\mathrm{COCH}_{3}\right), 14.0\left(\mathrm{CH}_{2} \mathrm{CH}_{3}\right) . \operatorname{HRESIMS}(\mathrm{m} / \mathrm{z}):[\mathrm{M}-\mathrm{H}]^{-}$calcd for $\mathrm{C}_{47} \mathrm{H}_{67} \mathrm{O}_{22} \mathrm{~N}_{2}$ 1011.4190, found 1011.4158. 
4. NMR data for compounds $3,25,30,31$ 
Compound 3, $400 \mathrm{MHz}, \mathrm{CDCl}_{3}$

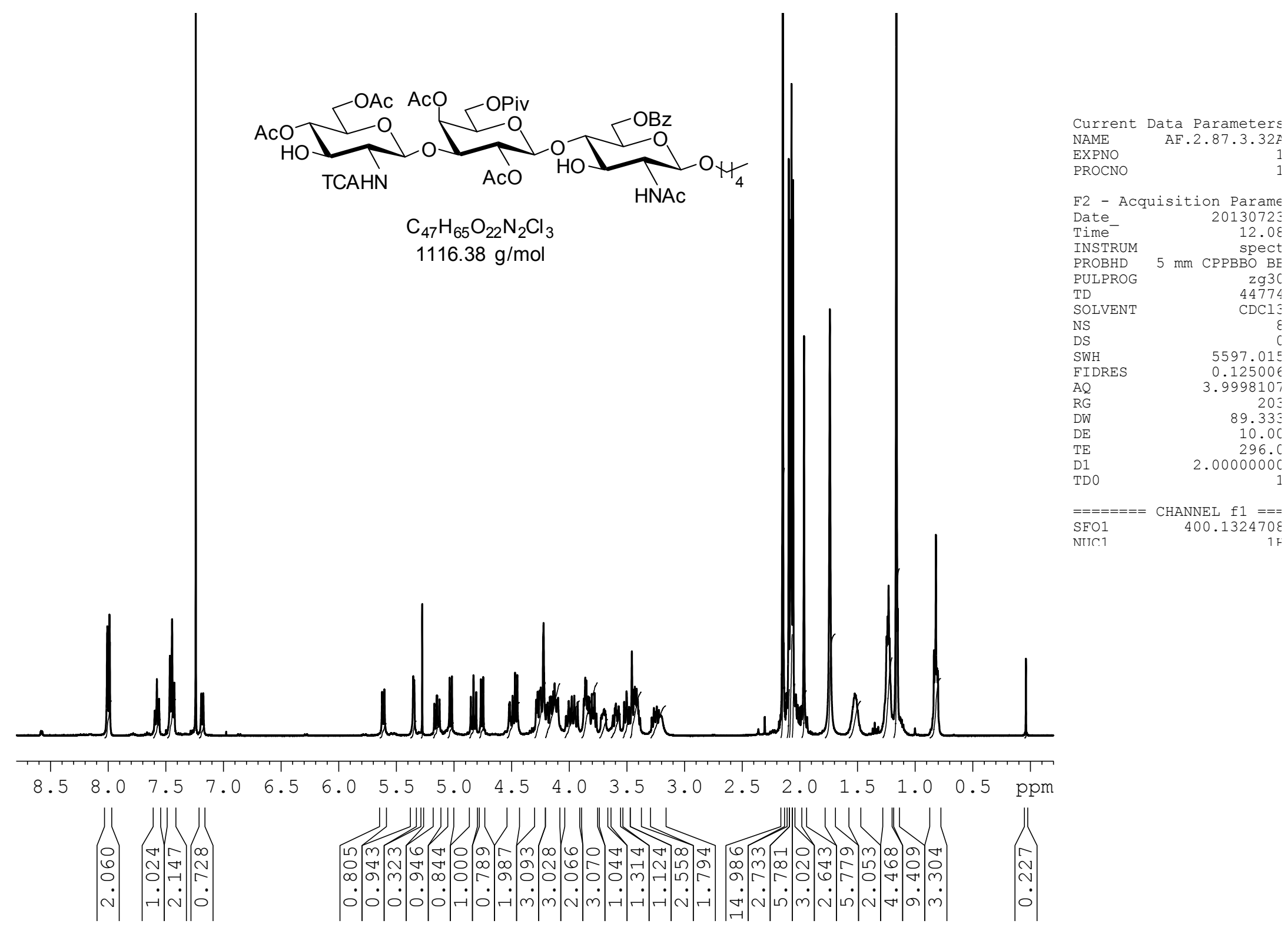


Compound 3, COSY, $400 \mathrm{MHz}, \mathrm{CDCl}_{3}$

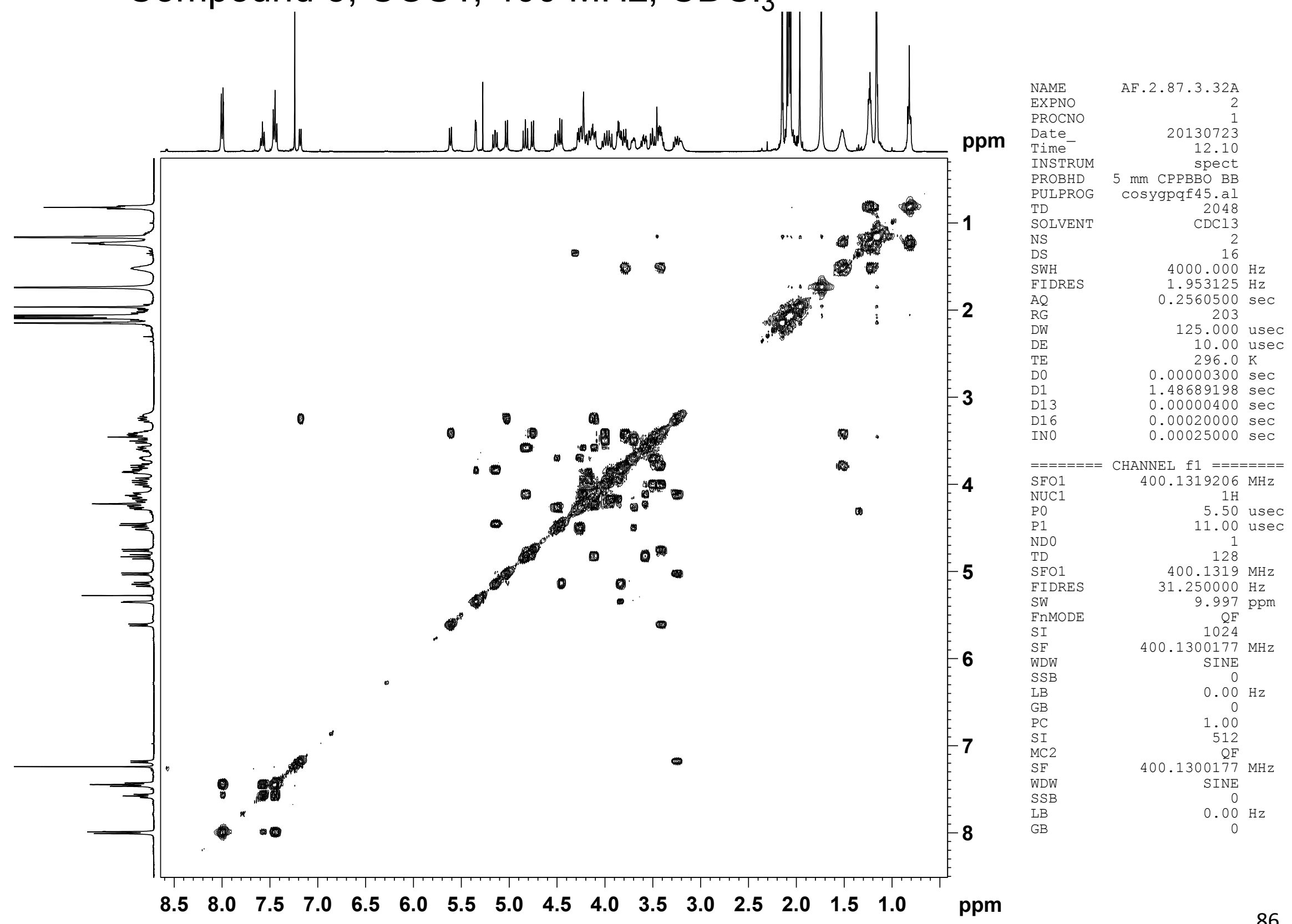




\section{Compound 3, $101 \mathrm{MHz}, \mathrm{CDCl}_{3}$}

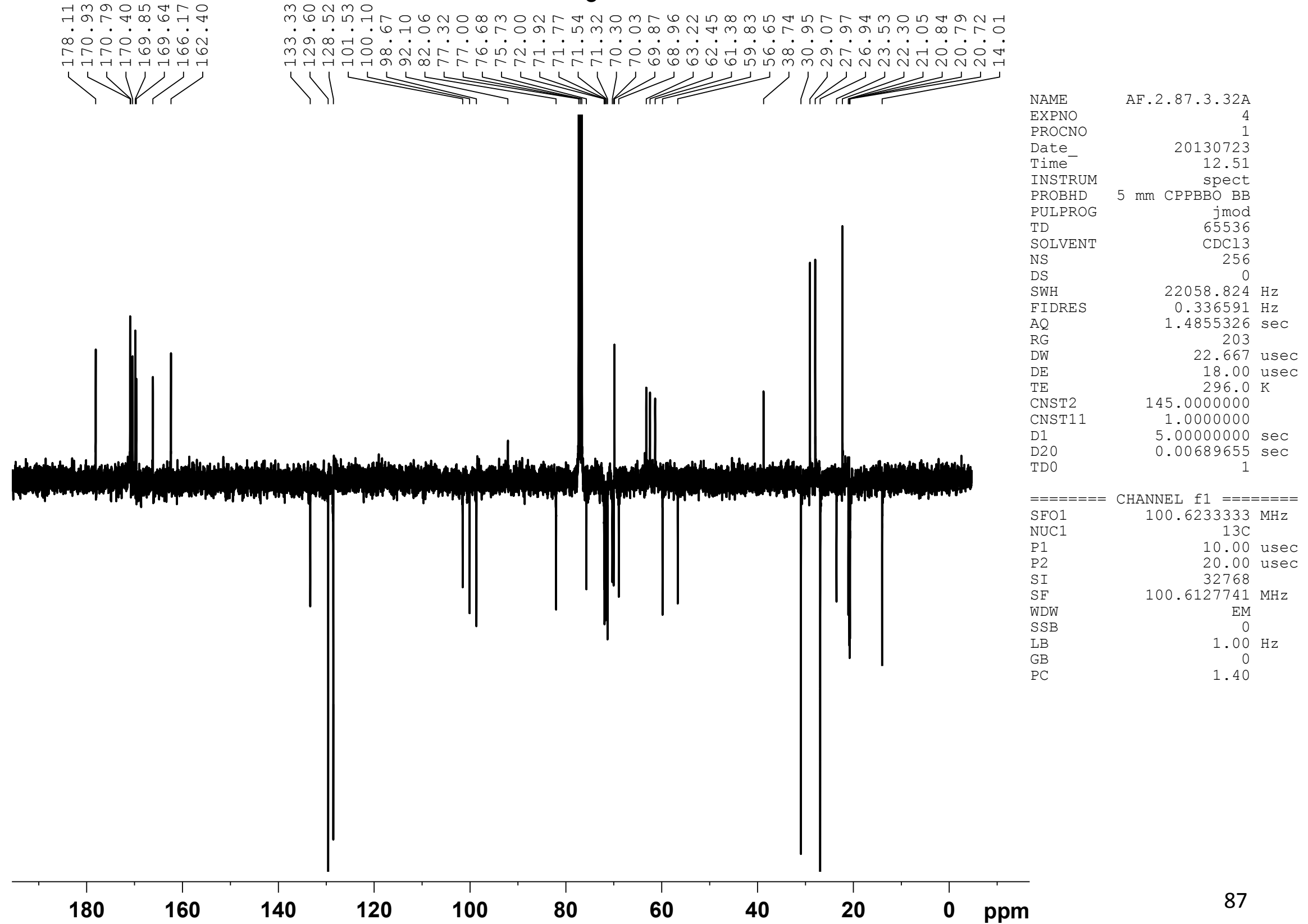


Compound 3, edited $\mathrm{HSQC}$ ( $\mathrm{CH}$ and $\mathrm{CH}_{3}$ blue, $\mathrm{CH}_{2}$ black), $400 \mathrm{MHz}, \mathrm{CDCl}_{3}$

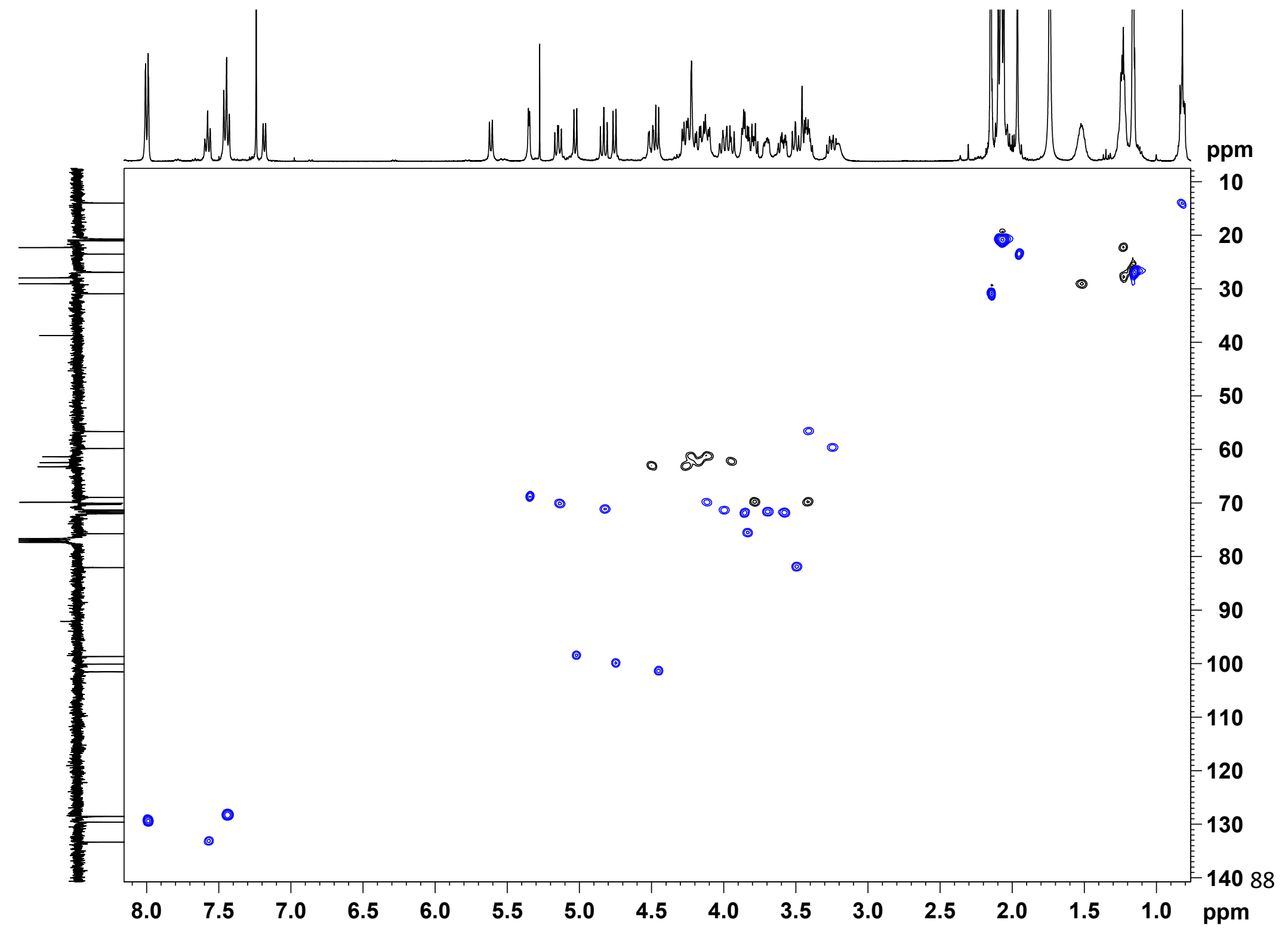


Compound 25, $600 \mathrm{MHz}, \mathrm{CDCl}_{3}$

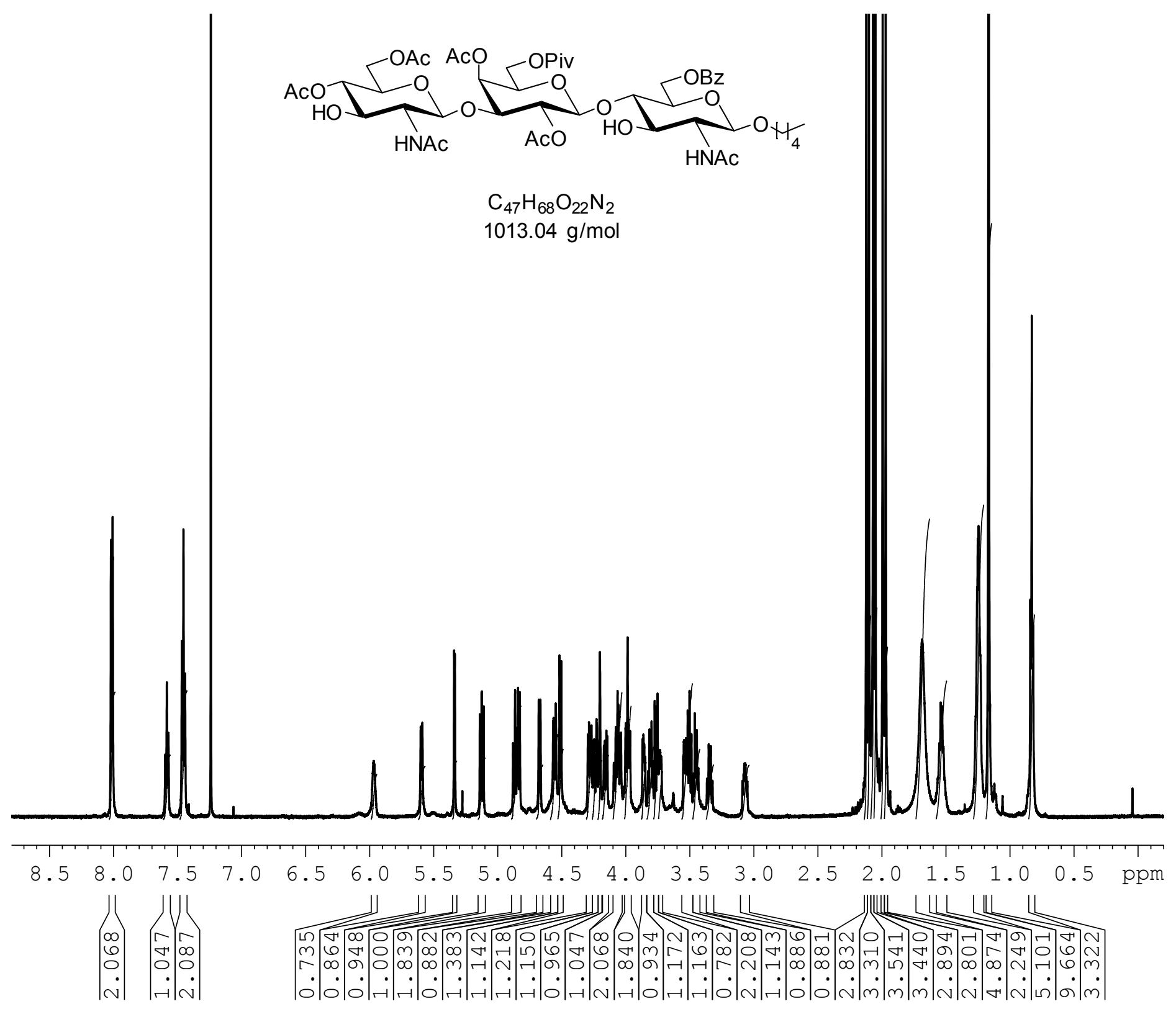

$\begin{array}{lr}\text { Current } & \text { Data Parameters } \\ \text { NAME } & \mathrm{AF} \cdot 3 \cdot 6.1 .44 \mathrm{~A} \\ \text { EXPNO } & 1 \\ \text { PROCNO } & 1\end{array}$

F2 - Acquisition Parame

Date 20130830

Time 10.33

Spect

$5 \mathrm{~mm}$ PATXO 19F

PUSC
PUPROG

SOLVENT

NS

$\begin{array}{lr}\text { DS } & \text { C } \\ \text { SWH } & 7812.50 \mathrm{C} \\ \text { FIDRES } & 0.11920 \mathrm{C}\end{array}$

$\mathrm{AQ}$
$\mathrm{RG}$

RW

DE

D1

$\mathrm{SFO} 1$
NITC1

CHANNEL $\mathrm{f} 1===$

$600.133600 \varepsilon$
$1 \mathrm{~F}$ 
Compound 25, COSY, $600 \mathrm{MHz}, \mathrm{CDCl}_{3}$

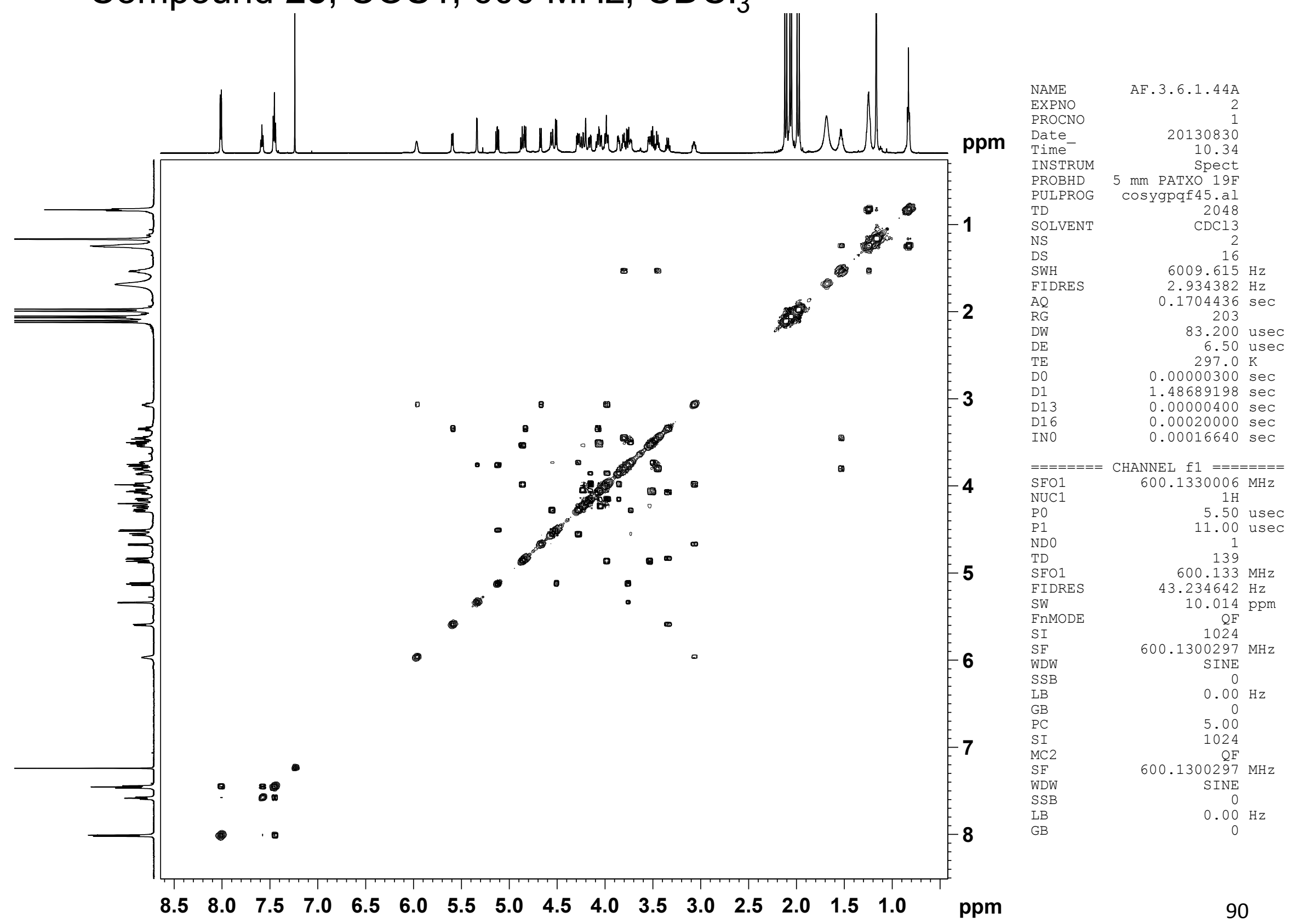




\section{Compound 25, $101 \mathrm{MHz}, \mathrm{CDCl}_{3}$}

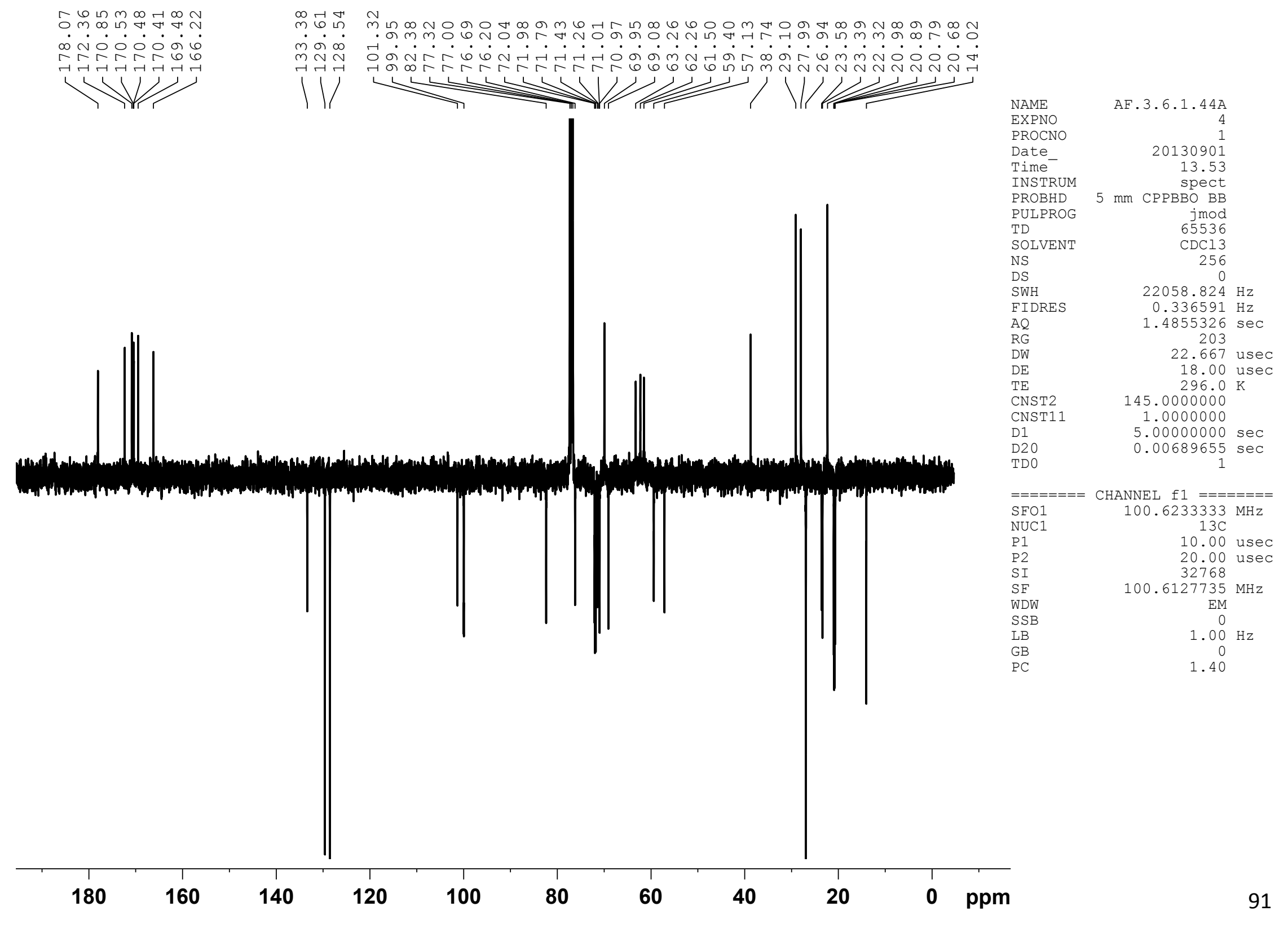


Compound 25, edited $\mathrm{HSQC}\left(\mathrm{CH}\right.$ and $\mathrm{CH}_{3}$ blue, $\mathrm{CH}_{2}$ black), $400 \mathrm{MHz}, \mathrm{CDCl}_{3}$

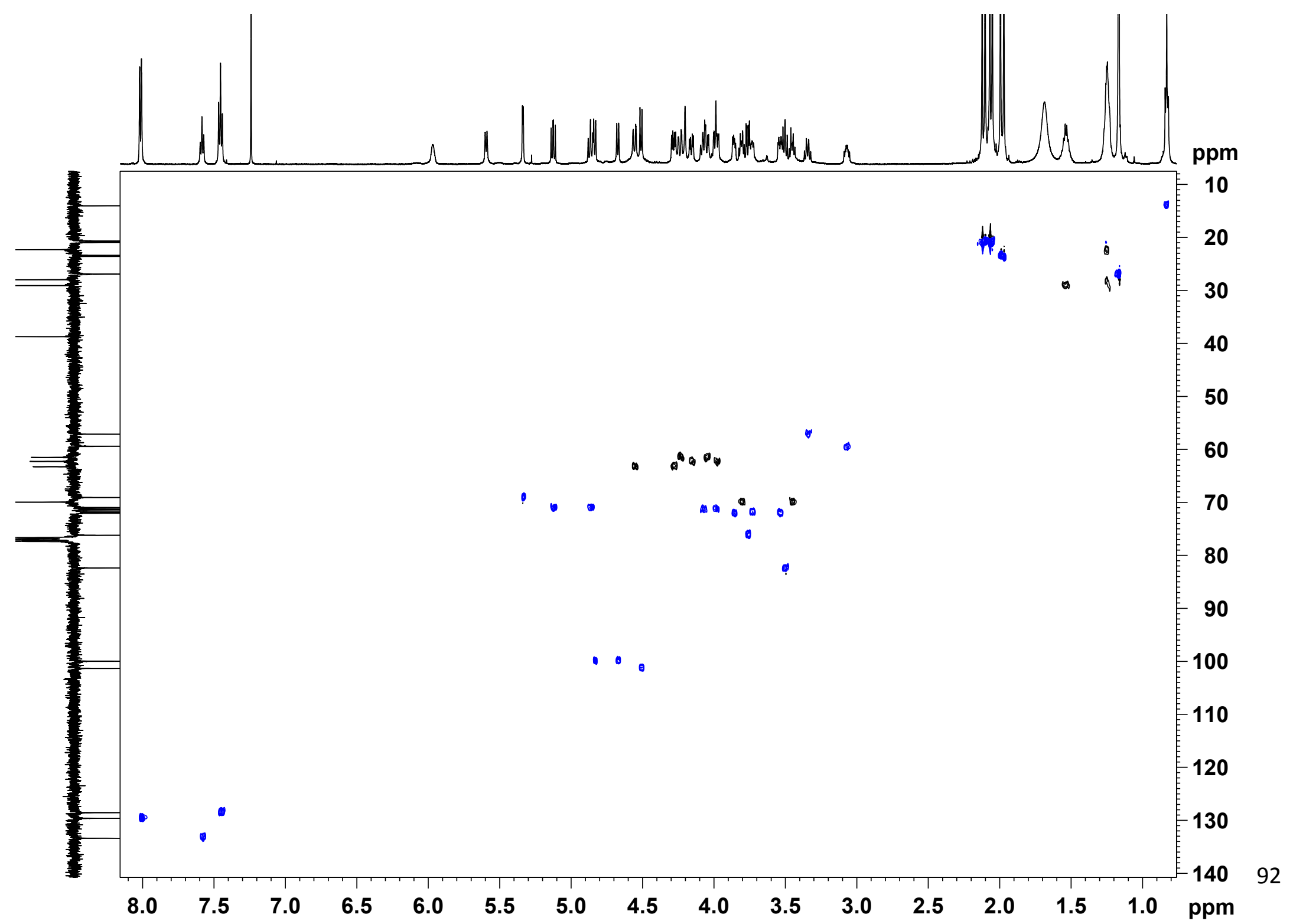




\section{Compound $30,400 \mathrm{MHz}, \mathrm{CD}_{3} \mathrm{OD}$}
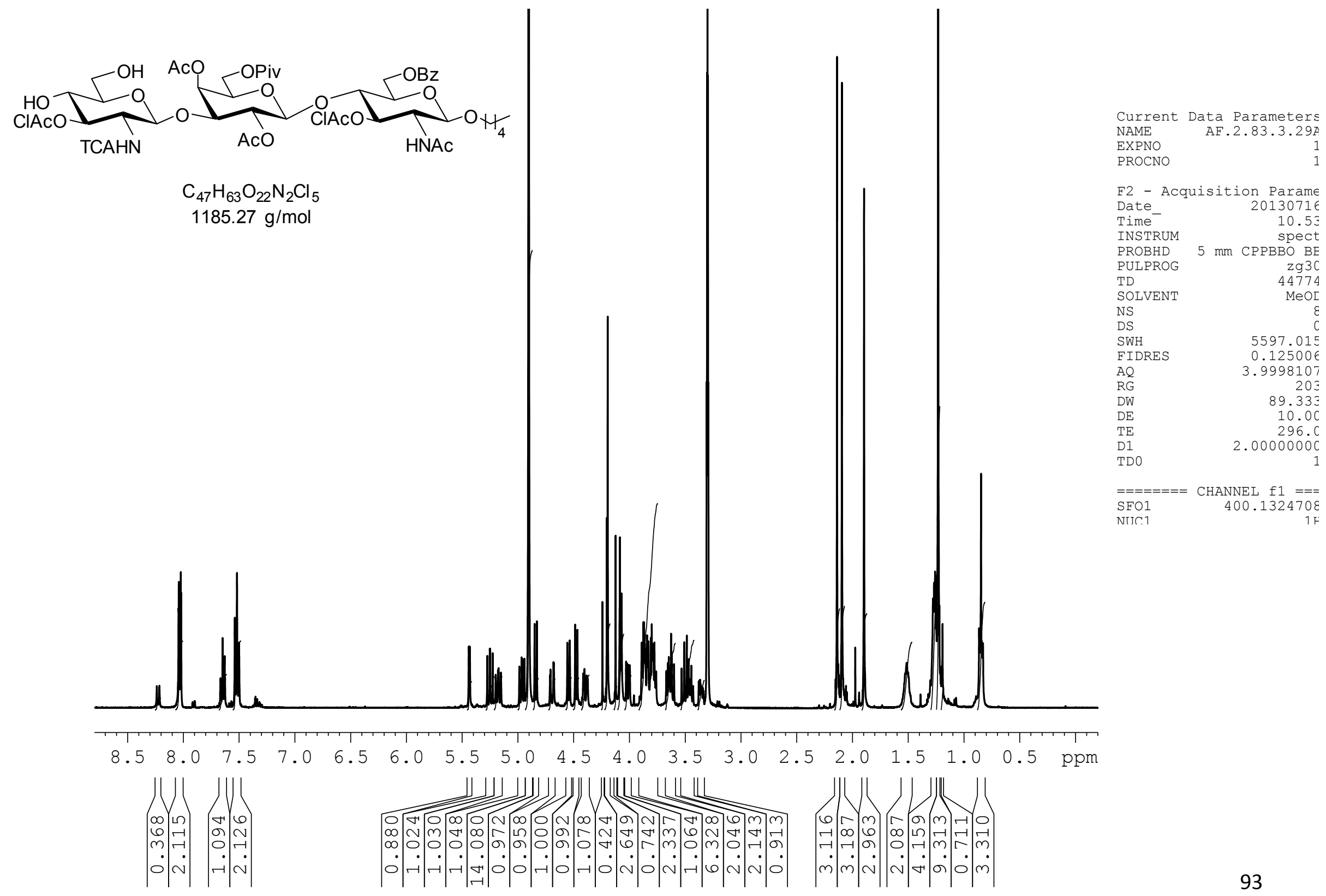
Compound 30, COSY, $400 \mathrm{MHz}, \mathrm{CD}_{3} \mathrm{OD}$

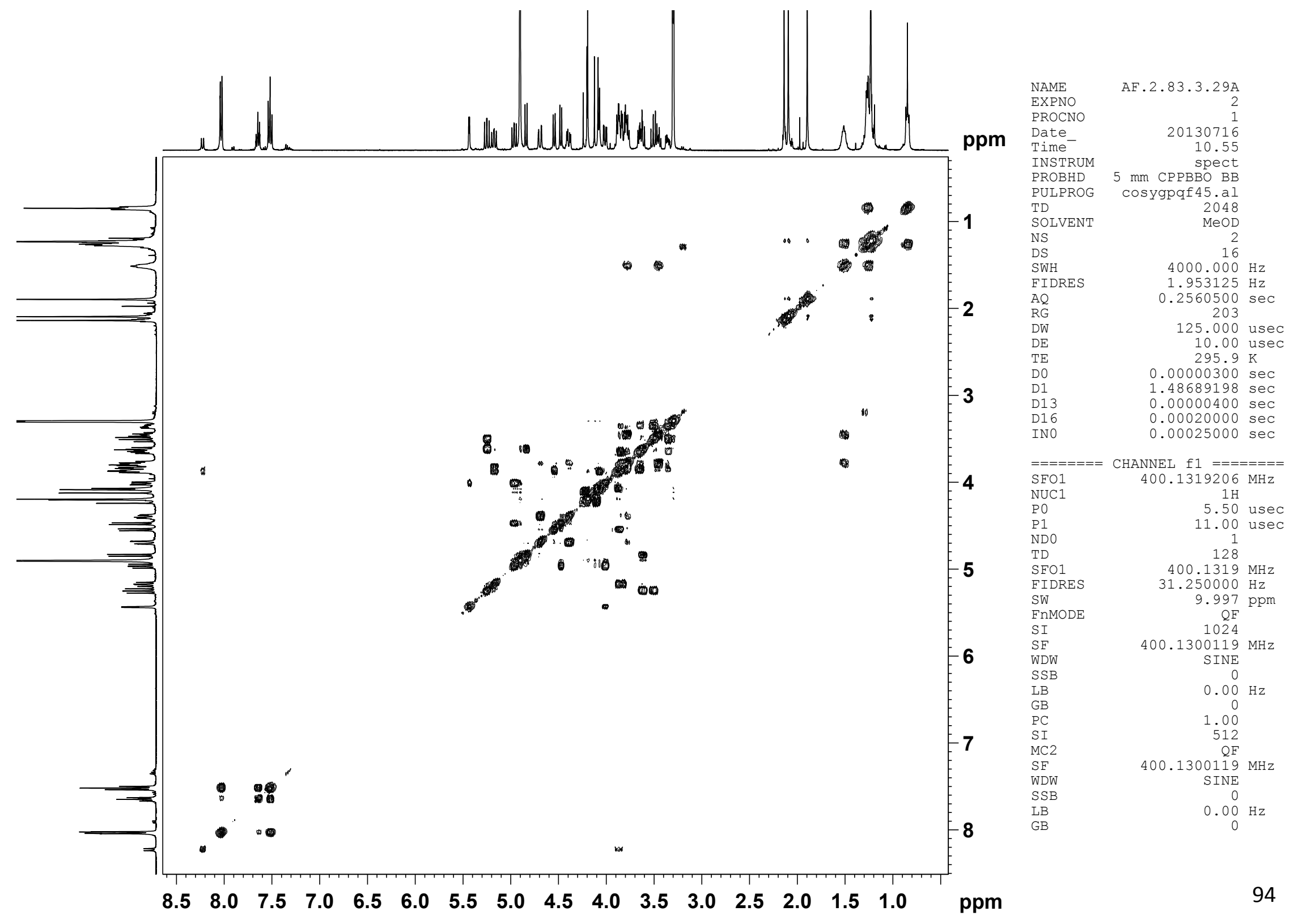




\section{Compound 30, $101 \mathrm{MHz}, \mathrm{CD}_{3} \mathrm{OD}$}

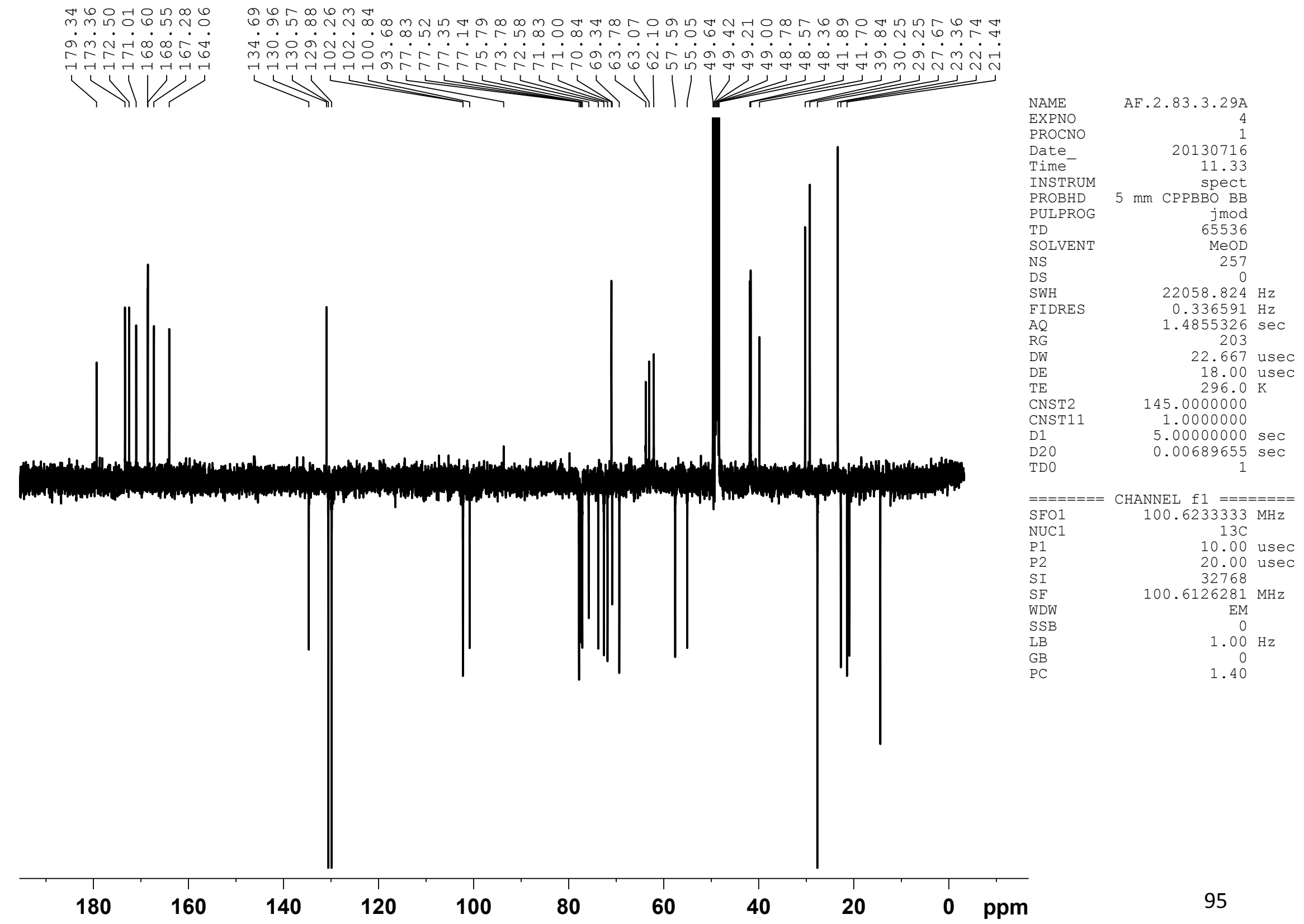


Compound 30, edited HSQC ( $\mathrm{CH}$ and $\mathrm{CH}_{3}$ blue, $\mathrm{CH}_{2}$ black), $400 \mathrm{MHz}, \mathrm{CD}_{3} \mathrm{OD}$

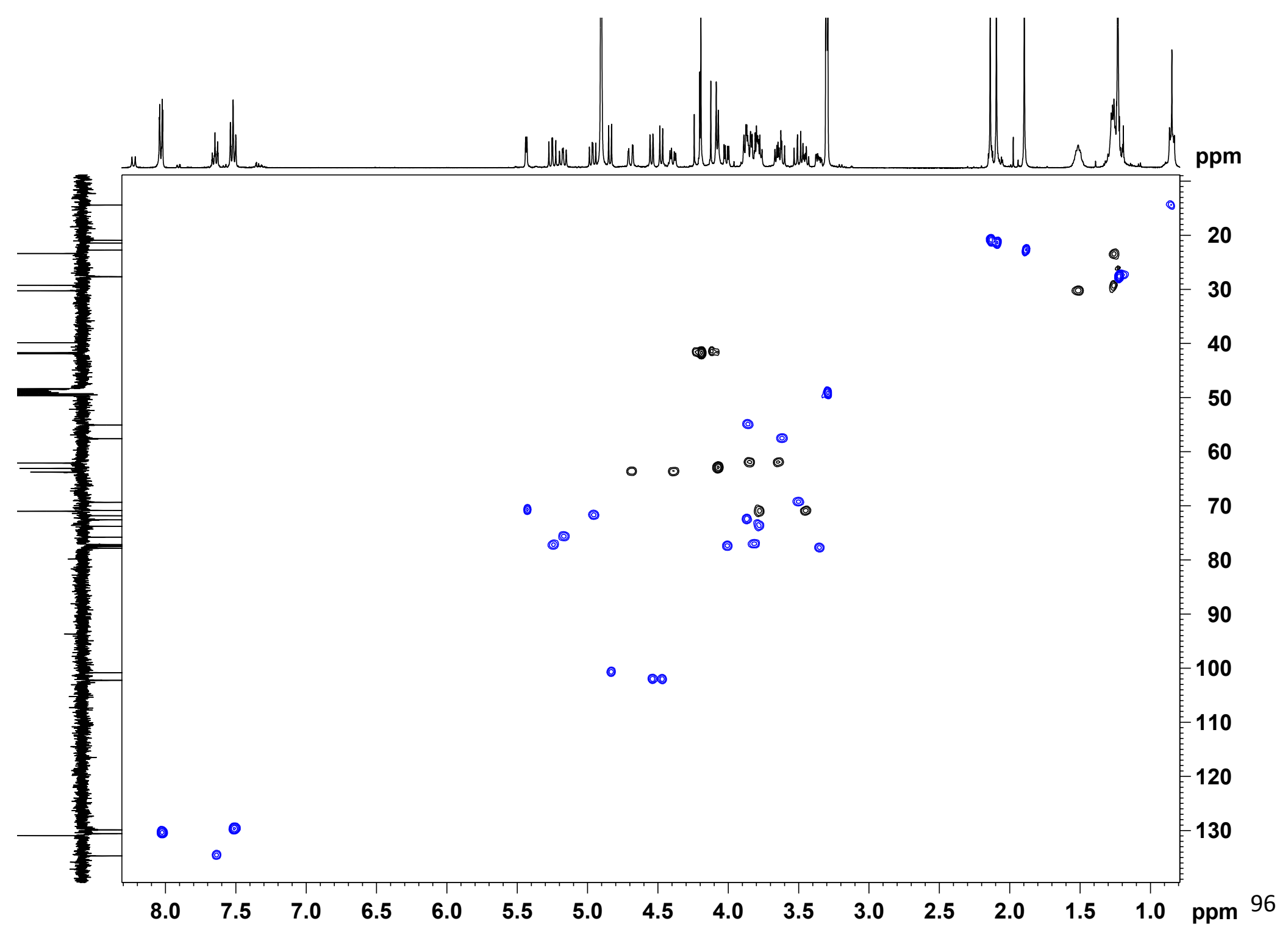




\section{Compound 31, $400 \mathrm{MHz}, \mathrm{CDCl}_{3}$}
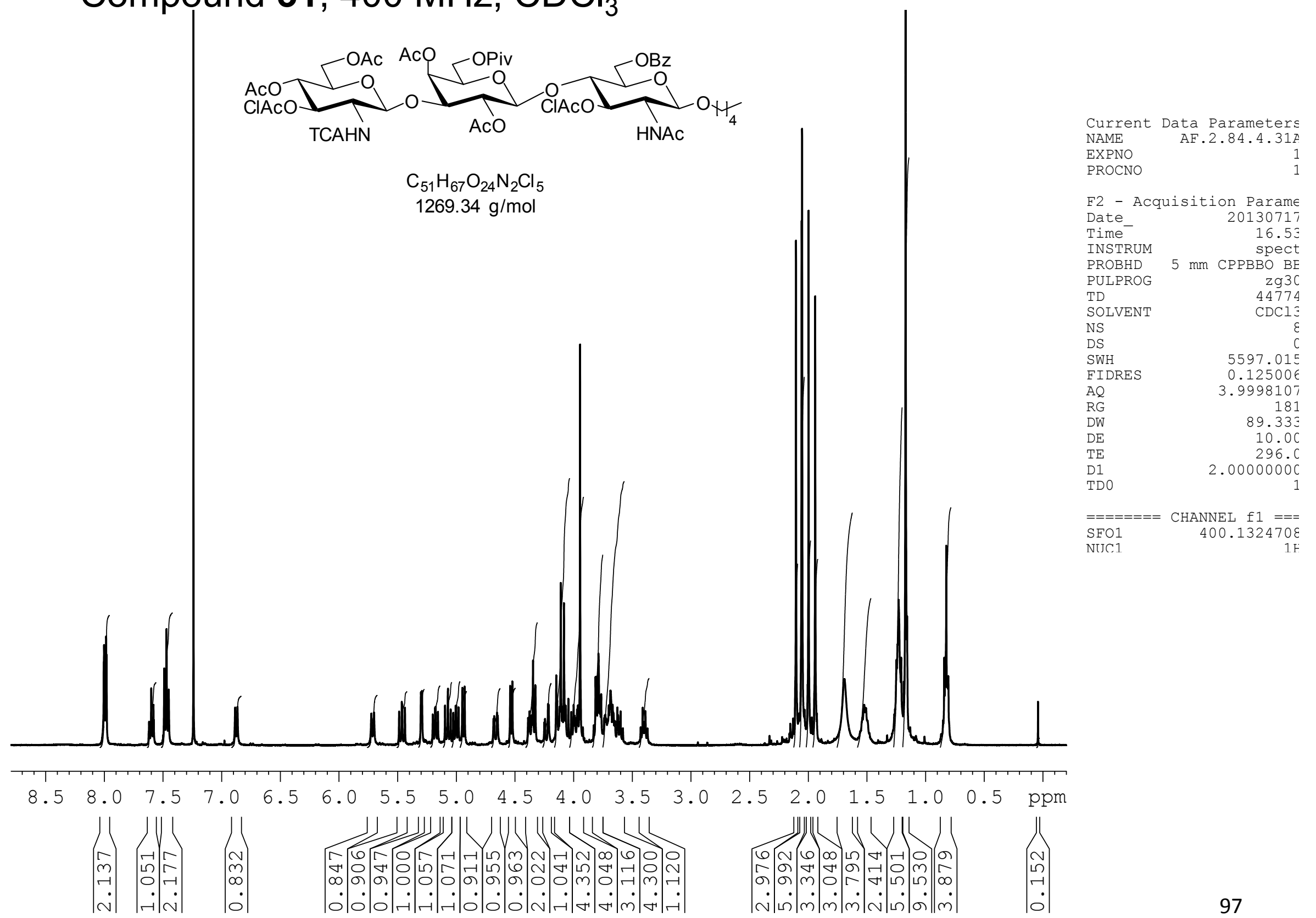
Compound 31, COSY, $400 \mathrm{MHz}, \mathrm{CDCl}_{3}$

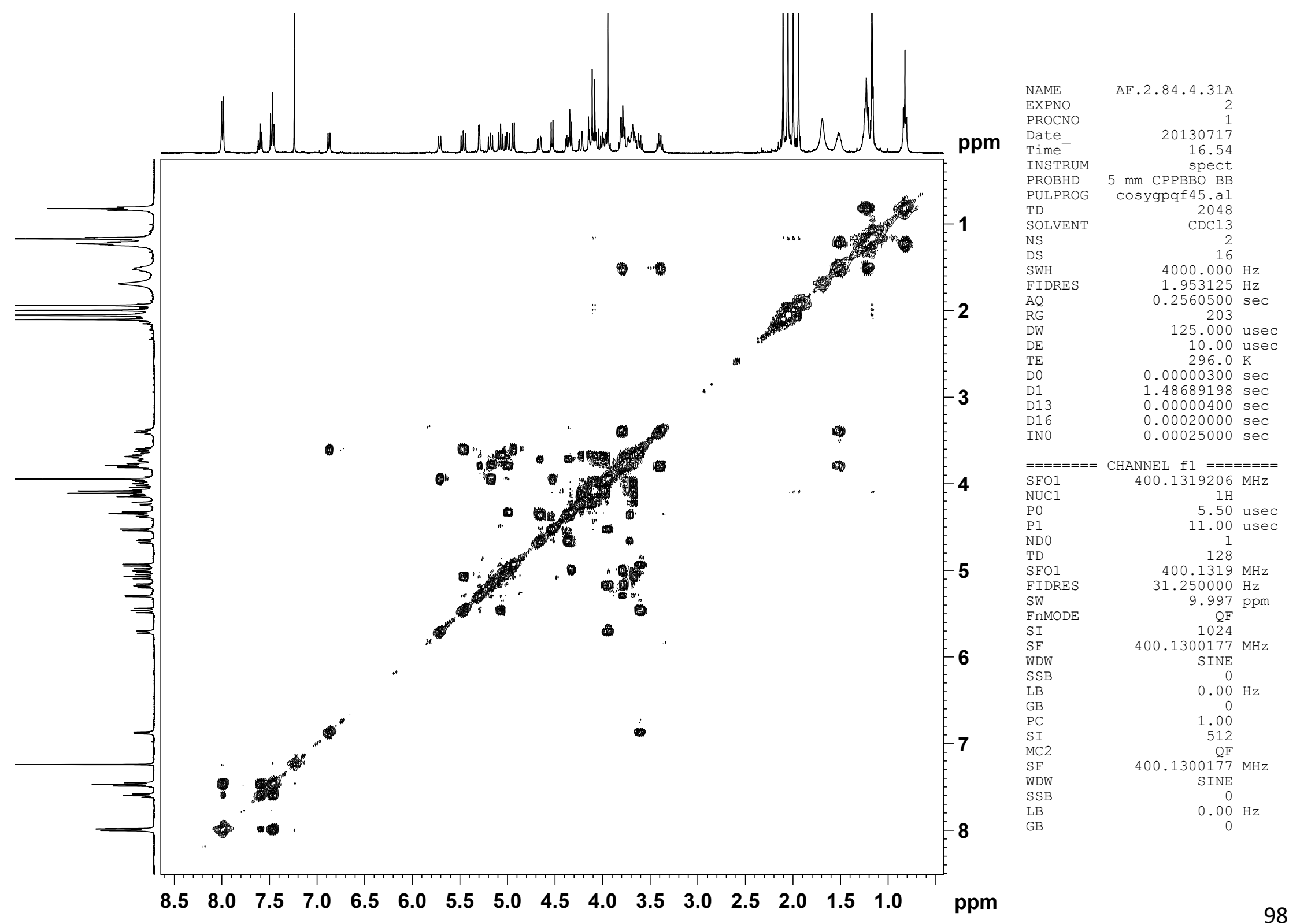




\section{Compound 31, $101 \mathrm{MHz}, \mathrm{CDCl}_{3}$}

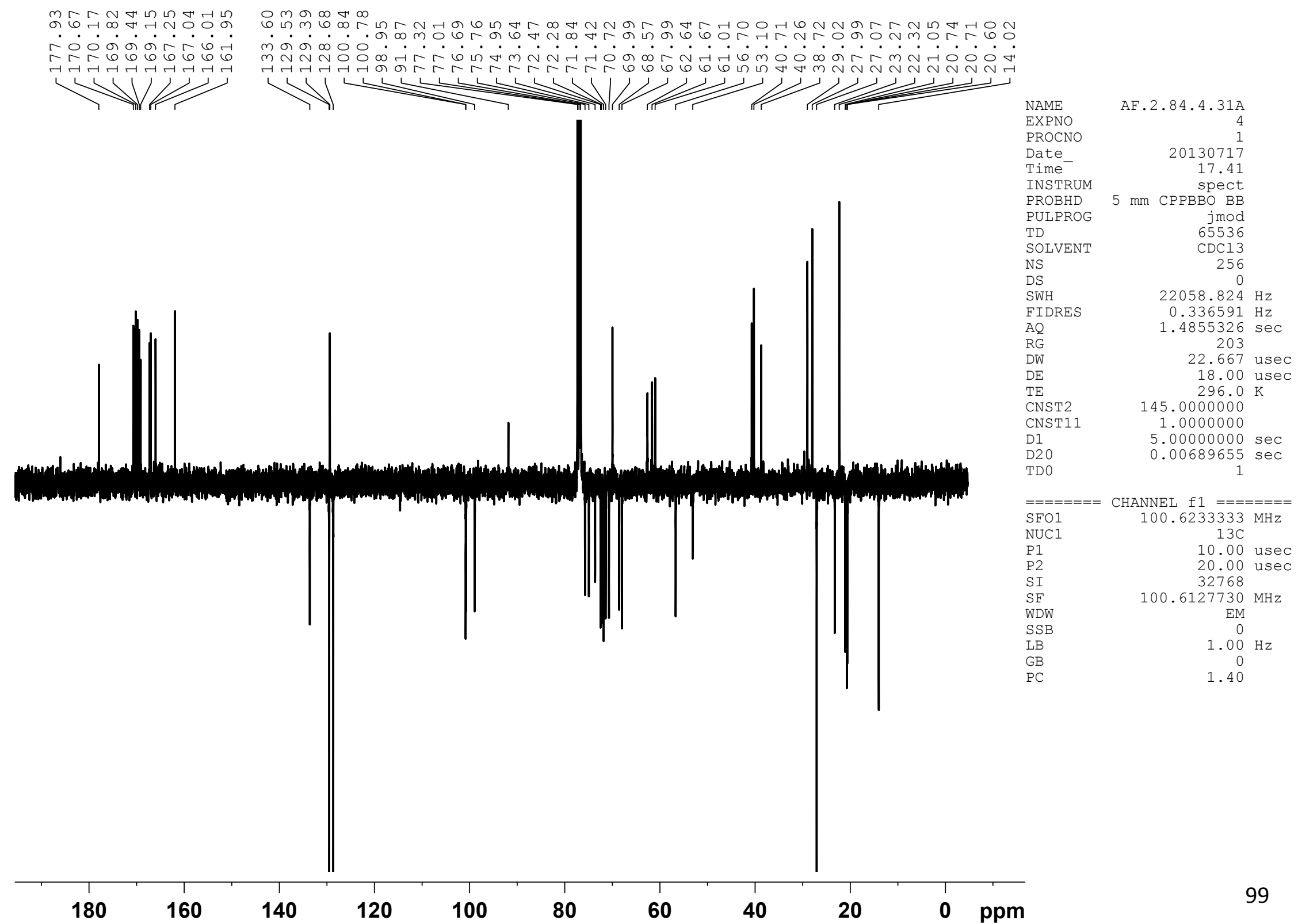


Compound 31, edited $\mathrm{HSQC}\left(\mathrm{CH}\right.$ and $\mathrm{CH}_{3}$ blue, $\mathrm{CH}_{2}$ black), $400 \mathrm{MHz}, \mathrm{CDCl}_{3}$

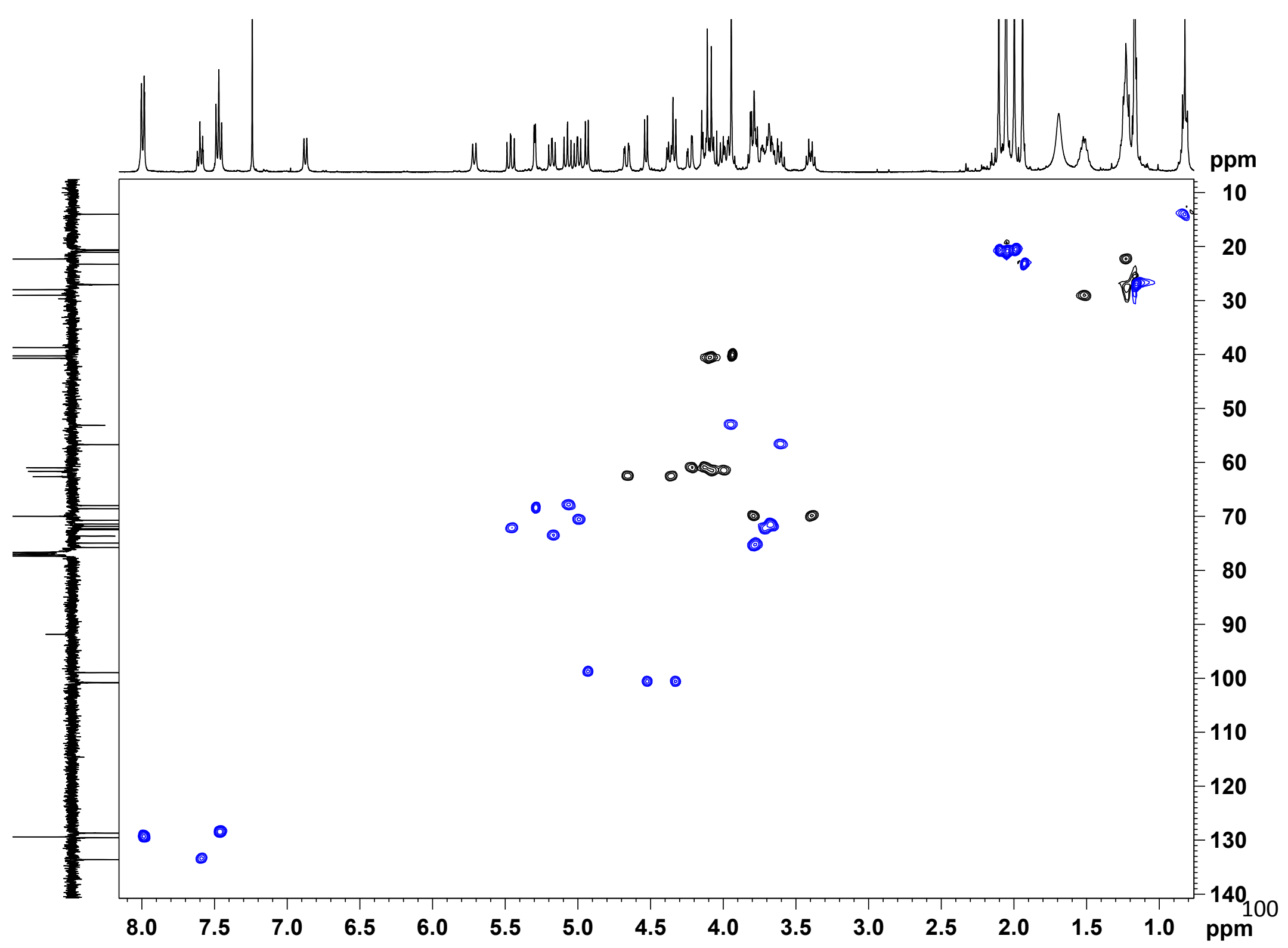

